\title{
Contributions to the knowledge of the Lepidoptera fauna of Southern Transylvania
}

\author{
Valeriu Albu \& Sebastian Albu
}

\begin{abstract}
Summary: We present here the data of the Lepidoptera species that we encountered in four Southern Transylvania (Romania) districts from 1966 to 2017. These were: Braşov, Covasna, Hunedoara and Sibiu. During this interval we identified 641 species in this area. Coleophora chalcogrammella, Wheeleria obsoletus, Hellinsia tephradactyla, Cadra cautella and Euchromius ocellea are first records for the region of Transylvania. We document the presence of the black locust leaf miner Parectopa robiniella from multiple, characteristic leaf mines found at Şinca Veche, Braşov, as well as that of the horse chestnut leaf miner Cameraria ohridella in Braşov, from muliple leaf mines and adult moths.
\end{abstract}

Keywords: Eastern European moths, invasive species, Lepidoptera leaf miners, Transylvania moths diversity

\section{Introduction}

Transylvania is a large region situated in the central part of Romania. It is a high plateau of a roughly triangular shape, bounded on the eastern and southern sides by the arch of the Carpathian Mountains and bordered in the west by the Apuseni Mountains. Administratively it is subdivided into ten districts: Bistriţa-Năsăud, Cluj, Sălaj, Alba, Mureş, Harghita, Covasna, Braşov, Sibiu and Hunedoara. The last three of these stretch from the heights of the Carpathian Mountains in the south, northward to the central plateau of Transylvania. Covasna is situated east of these three and is nestled in the arch of the Carpathian chain.

Transylvania has a long history of lepidopterological research, dating back to the mid-19th century (SzÉKeLY, 2014). Fuss (1850) published the first catalog of the lepidoptera from Transylvania which lists 965 species and subspecies from its southern part. Today Transylvania is the best studied area of Romania, with 2985 lepidoptera species recorded (RÁkosY et al., 2003). The variety of geographical features and vegetation helps explain the lepidoptera species diversity in the region. The western opening over the generally low elevation Apuseni Mountains keeps this region in contact with the central European fauna, while the mountain passes through the Carpathian range allow an influx of species from the Wallachian Plain in the south and the Moldavian Plain in the east.

\section{Material and methods}

We sampled various localities in the four southern Transylvanian districts of Covasna, Braşov, Sibiu, and Hunedoara between 1966 and 2017 (Table 1). In these districts we investigated areas of low to medium elevation, usually under $1000 \mathrm{~m}$ in altitude and also three sites in Braşov that were at higher elevation: Fundata village $(1290 \mathrm{~m})$ and the peaks of Postăvarul and Piatra Mare $(2000 \mathrm{~m})$. The districts were not sampled equally. Eighteen sites were sampled in Braşov, three each in Covasna and Hunedoara, and a single site in Sibiu.

For day sampling we used an insect net to catch specimens that were nectaring on flowers or perched on vegetation. Occasionally, we found larvae that we reared on either the plant on which they were found or on dandelion (Taraxacum sp.) or plantain (Plantago sp.) leaves. Night time sampling was conducted using a 160 watt ultraviolet portable tanning reflector lamp to attract specimens and by inspecting mercury vapor bulbs installed on permanent structures in suitable vegetation areas. Desirable specimens were introduced into a jar with a gaseous ammonia atmosphere produced from ammonia carbonate crystals. Large individuals were injected with liquid ammonia in the thorax for a quick dispatch.

We used „The Lepidoptera of Europe” checklist (KARSHOLT \& RAZOWSKI, 1996) and „Catalogul Lepidopterelor României” (RÁkosy et al., 2003) to check for previous records of a taxon for our area. We used the site http://fauna-eu.org (KARSHOLT \& 
NiEUKERKEN, 2013) for more recent taxonomical updates. In the 22 years since its publication, „The Lepidoptera of Europe" checklist (KARSHOLT \& RAZOWSKI, 1996) has undergone numerous changes at subgeneric as well as suprageneric levels. These changes reflect the tendency to order the various lepidoptera groups by more meaningful, phylogenetic criteria. More recent classifications are largely based on nucleotide sequence data. However, this re-classification of the taxa also caused a change in the position of many species, genera, and groups of genera, leading to a major disturbance of the initial numbering sequence of the species in the checklist, thus making it difficult to find a species by its allocated sequential number. Consequently, we used the more updated classification from KARSHOLT \& NiEUKERKEN, 2013, but kept the old numbering from the 1996 checklist to facilitate the species' recognition in their new positions.

\section{Results}

A total of 1662 lepidoptera specimens representing 641 species in 20 superfamilies were recorded during this study. 1011 specimens were collected from

Table 1 . The investigated sites by district.

\begin{tabular}{cc}
\hline District & Locality \\
\hline Braşov & Braşov \\
& Drăuşeni \\
Fundata & Homorod \\
Pădurea Bogata & Piatra Mare Mt. \\
& Poiana Braşov \\
& Postăvar Mt. \\
& Râşnov \\
& Roadeş \\
& Săcele \\
& Sâmbăta de sus \\
& Sânpetru \\
& Şinca veche \\
& Tâmpa Mt. \\
& Teliu \\
& Vlădeni \\
& Voila \\
& Bodoc Mt. \\
Sibiu & Breţcu \\
& Vâlcele \\
& Beriu \\
& Costeşti \\
& Sibişel \\
& Richiria \\
\hline
\end{tabular}

Braşov, comprising 413 species, 204 of which were caught inside the perimeter of the city proper. We also recorded 467 specimens comprising 264 species from Hunedoara and 176 specimens comprising 98 species from Covasna. Eight specimens corresponding to 7 species were collected from Sibiu. The most speciesrich superfamilies were Noctuoidea and Geometroidea with 173 and 123 species, respectively (Tables 2 and 3).

Five species were added as new records to the fauna of Transylvania: Coleophora chalcogrammella ZELLER, 1839, Wheeleria obsoletus (ZELLER, 1841) Hellinsia tephradactyla (HüBNER, 1813) from Braşov, Cadra cautella (WALKER, 1863), and Euchromius ocellea (HAWORTH, 1811) from Hunedoara.

Seven species were confirmations of rare or questionable recordings in the Transylvanian fauna: Bryotropha simils (StAINTON, 1857), Gelechia sabinellus(ZELLER, 1839), Marasmarchalunaedactyla (Haworth, 1811), Phiaris palustrana (Lienig \&

Table 2. Species and specimens recorded for each district.

\begin{tabular}{ccc}
\hline District & $\begin{array}{c}\text { Number of } \\
\text { species }\end{array}$ & $\begin{array}{c}\text { Number of } \\
\text { specimens }\end{array}$ \\
\hline Braşov & 413 & 1011 \\
Hunedoara & 264 & 467 \\
Covasna & 98 & 176 \\
Sibiu & 7 & 8 \\
Total & 641 & 1662 \\
\hline
\end{tabular}

Table 3. Superfamilies with their number of species encountered in this study.

\begin{tabular}{cc}
\hline Superfamily & Number of species \\
\hline Micropterigoidea & 1 \\
Hepialoidea & 2 \\
Adeloidea & 5 \\
Tineoidea & 6 \\
Gracillarioidea & 3 \\
Yponomeutoidea & 15 \\
Gelechioidea & 29 \\
Pterophoroidea & 13 \\
Choreutoidea & 1 \\
Tortricoidea & 59 \\
Cossoidea & 8 \\
Zygaenoidea & 11 \\
Thyridoidea & 1 \\
Pyraloidea & 72 \\
Papilionoidea & 92 \\
Drepanoidea & 8 \\
Lasiocampoidea & 7 \\
Bombycoidea & 12 \\
Geometroidea & 123 \\
Noctuoidea & 173 \\
\hline
\end{tabular}




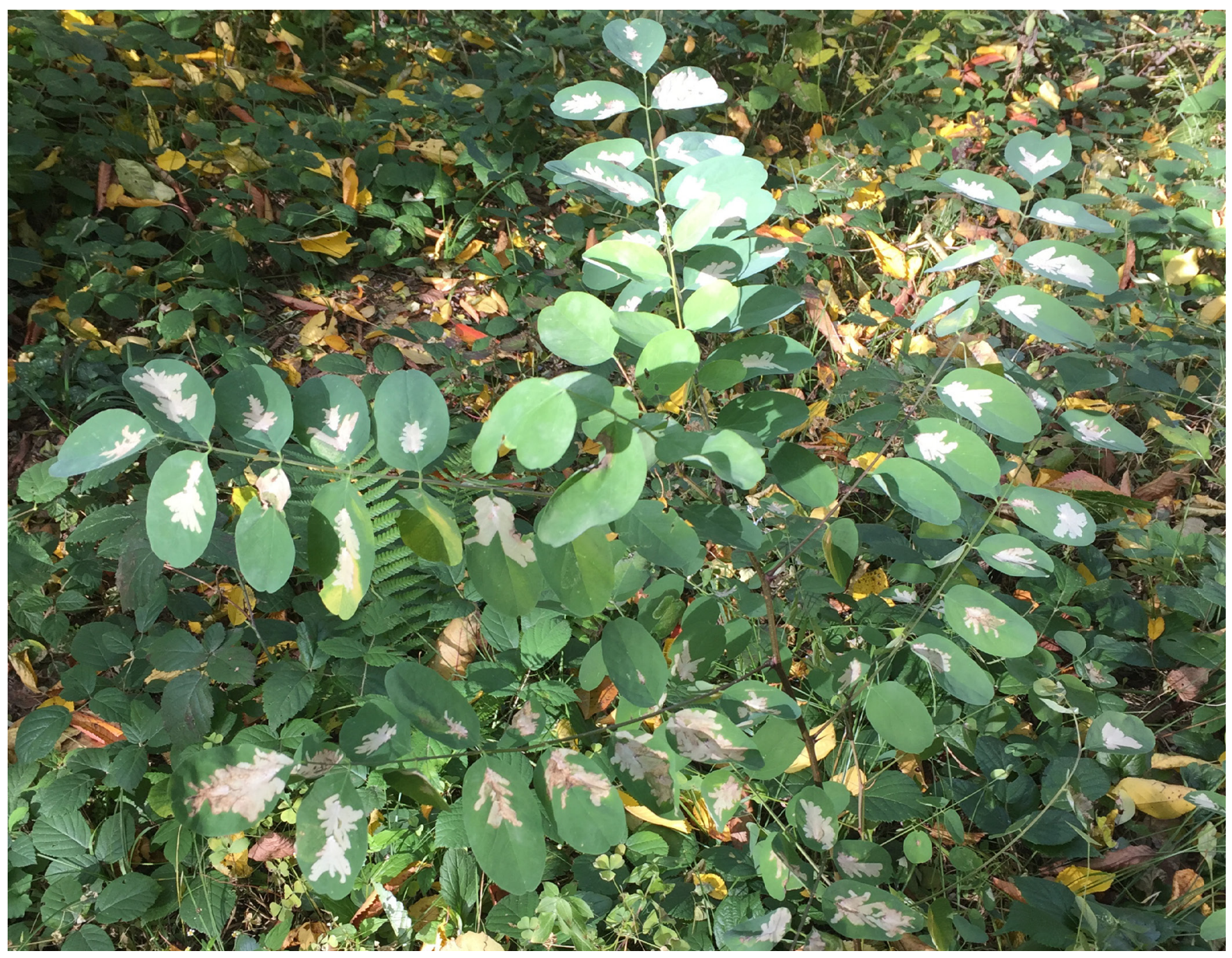

Fig. 1. Robinia pseudacacia leaf infestation with leaf mines highly suggestive of Parectopa robiniella at Şinca Veche, Braşov.

ZELLER, 1846), Eucosma lacteana (TREITSChKE, 1835), Dichrorampha eximia (DANILEVSKy, 1948), and Buszkoiana capnodactylus (ZeLLER, 1841).

We found many residual leaf mines on black locust trees near Şinca Veche in Braşov with their characteristic shape strongly suggesting the presence of Parectopa robiniella (CLEMENS, 1863) in the area (Fig. 1), but we did not collect the adult moth.

Appendix 1 lists all the species recorded during this study in all the investigated localities.

\section{Discussion}

The 641 species recorded in this study represent $21 \%$ of the 2985 species known from Transylvania (RÁKosy et al., 2003).

Of the seven species with previous uncertain status in Transylvania, M. lunaedactyla and E. lacteana were recorded as questionably present in an earlier list (Popescu-GorJ, 1984). According to the same list, the others (B. similis, G. sabinellus, P. palustrana, D. eximia, and $B$. capnodactylus) were considered absent from the fauna of Transylvania (and Romania), until they were added in a more recent catalog (RÁKOSY et al., 2003). Another species from our records was

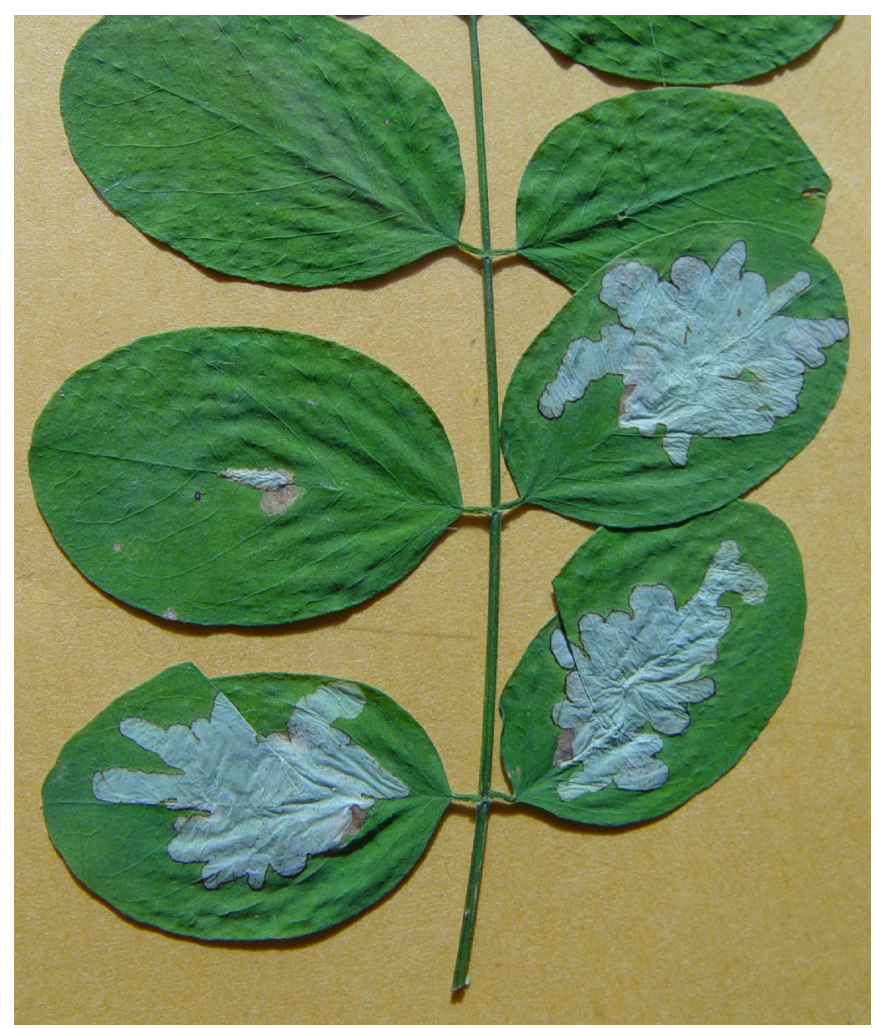

Fig. 2. Close-up of the mines in Robinia pseudacacia leaflets at Şinca Veche, Braşov. 
previously known from Transylvania under a different name. Dichrorampha gueneeana OBRAztsov, 1953 was changed to $D$. vancouverana McDunnuogh 1935, the former being a junior synonym of the latter (Miller, 1999). This moth has been long-known from the Palearctic region, as attested to by many old records, but was not properly described and named until a North American immigrant specimen was captured on Vancouver Island, Canada,. The species is established on both western and eastern coasts of North America (MilLER, 1999).

We document the presence of Paranthrene tabaniformis (RoTTEMBURG, 1775) from Transylvania. This species was known to occur in Romania, as it appeared in an earlier catalog (POPESCU-GoRJ, 1984), albeit as $P$. synagriformis (RAMBUR, 1866). This latter taxon is a subspecies of $P$. tabaniformis. The name was omitted in the 2003 list (RÁKOsY et al., 2003), but was re-added in the 2006 Additions and Corrections to the list of the Lepidoptera of Romania (RÁKOSY \& GoIA, 2006).

We also found traces of a member of the NorthAmerican fauna that is spreading over Europe, according to a recent map published by KARSHOLT \& NiEUKERKEN, (2013). We observed heavy infestations of leaf mines on black locust, Robinia pseudoacacia L. (Fabaceae) in a forest near Şinca Veche in Braşov district. The large, irregular, digitate mine occurs on the upper surface of the leaflet (Fig. 2) and is characteristic of Parectopa robiniella (CLEMENS, 1863) (Gracillariidae). This species was first recognized in the Romanian fauna in 1995 in the southern part of the country and has since also been recorded from Transylvania (RÁkosy et al., 2003). We chose to include this taxon in our list despite the lack of a voucher specimen because we consider that the many characteristic mines found at Sinca Veche strongly points toward the moth's presence in that area. The host itself, the black locust, was introduced to Europe from North America in the seventeenth century and has spread unchecked through Europe, reaching Romania in the mid-eighteenth century (ENESCU, 2014). In the latter part of the twentieth century two of the several black locust feeders, $P$. robiniella and Macrosaccus robiniella (CLEMENS, 1859) (Gracillariidae) appeared in Europe (KARSHOLT \& Nieukerken, 2013). The latter was first reported in Romania in 2004 by Kovács et al. (2006). While we did find mines that attest to the presence of $P$. robiniella in our study area, we did not find mines of $M$. robiniella, which are rounded blotches on the underside of the leaf.

During 2013-2017 we also observed widespread leaf mine infestations on horse chestnut, Aesculus hippocastanum L. (Sapindaceae), a widely cultivated ornamental tree in the streets and urban parks of Braşov These infestations indicated the presence of Cameraria ohridella Deschka \& Dimić, 1986 (Gracillariidae), previously described from Macedonia. The moth was present in the southern Balkan Peninsula since at least 1879 , as shown by the DNA barcoding of larvae of this insect preserved in pressed herbarium leaf samples (LEES \& al., 2011). However, beginning in the late 1980s it started a sudden and rapid expansion into Europe, also invading Romania (ŠEFrové \& LAŠTŮVKA, 2001). We found mines and adults of $C$. ohridella on horse chestnut leaves in June 2002 in the Northern Transylvanian city of Gherla. In Braşov, virtually the entire horse chestnut population of the city is being infested year after year with multiple mines detected in each leaf (personal observation). Despite this stress, the trees appear to be withstanding the attack by budding, flowering and bearing fruits every year.

The city of Braşov is a sprawling urban community surrounded by extensive agricultural lands. Through its geographical position it is spread across several hills and is encircled by many more, some of them leading uninterrupted to high elevation mountain ranges. These hills are still heavily wooded in areas between anthropic developments and generally are connected amongst each other and with the surrounding mountains. Even though we did not set out to investigate the effects of the urbanization process on the city's lepidopterological fauna, we recognized the diversity of this urban fauna. We recorded 204 different species from the city and its urbanized hills. In comparison, a previous study of the lepidoptera richness in an urban park of Bucureşti (Albu \& Albu, 2016), recorded 170 species from that setting, $17 \%$ less than from Braşov.

The Lepidoptera are a dynamic group whose distribution changes under various climate and vegetation-related influences. The relationships between $P$. robiniella and $C$. ohridella and their hosts illustrate the ongoing dynamism between some lepidoptera and their plant hosts, and similar symbioses will probably continue to be uncovered by future research.

\section{Aknowledgements}

We gratefully recognize the help of Cees GiELIS in identifying the Romanian Pterophoridae for a previous paper (ALBU, 2009) and that of Jean-François LANDRY for identifying the Coleophoridae and Scythrididae collected during this study. Lászlo RÁKosy helped us clarify the identity of several uncertain taxa. We also acknowledge the contribution of the late Dr. Tănase BALAş to the information contained herein by providing the entire Sibiu district and many of the Hunedoara district data.

\section{References}

Albu, V. 2009.Contributions to the knowledge of the Pterophoridae (Lepidoptera) fauna of Romania. Entomologica Romanica 14: 23-28.

Albu, V., Albu, S. 2016. Contributions to the knowledge of the Lepidoptera species diversity in an urban park 
setting of Bucharest, Romania, with considerations on the species dynamics in the city over the last century. Entomologica Romanica 20: 69-78.

ENESCU, M. 2014. Trees of Romania. http://maimultverde. ro/salcamul-in-romania

Fuss, C. 1850. Verzeichnis der bis jetzt in Siebenbürgen aufgefundenen Lepidopteren. [Catalog of the Transylvanian Lepidoptera as known so far]. Mitteilungen siebenbürgischen Vereines der Naturwissenschaft. Hermannstadt. 1:54-64. In German.

Karsholt, O., Razowski, J. (ed.) 1996. The Lepidoptera of Europe. A Distributional Checklist. Apollo Books. Stenstrup, Denmark.

Karsholt, O., Nieukerken, E. J. van 2013. Lepidoptera, Moths. Fauna Europaea version 2017.06, http://faunaeu.org

Kovács, Z., Kovács, S., SzabóKy, Cs. 2006. The occurrence of Phyllonorycter issikii (KUMATA, 1963) and Phyllonorycter robiniella (Clemens, 1859), two invasive leaf miner species in the fauna of Romania (Lepidoptera, Gracillariidae). Entomologica romanica, 11:5-7.

Lees, D.C., LaCK, W.H., Rougerie, R., HernandeZ-Lopez, A., Raus, T., Avtzis, N.D., Augustin, S., LopezVAAMONDE, C. 2011. Tracking origins of invasive herbivores through herbaria and archival DNA: the case of the horse-chestnut leaf miner. Frontiers in Ecology and the Environment. Vol. 9, Issue 6, pages 322-328.

Miller, W.E. 1999. A new synonymy in Dichrorampha that reveals an overlooked immigrant record for North America (Tortricidae). Journal of the Lepidopterists' Society 53(2), 74-75.

Popescu-GorJ, A. 1984. La liste systématique des espèces de Microlépidoptères signalées dans la faune de Roumanie. Mise à jour de leur classification et nomenclature [Systematic catalog of the Microlepidoptera species recorded in the fauna of Romania. Update of their classification and names]. Travaux du Muséum d'Histoire Naturelle „Grigore Antipa”. 26: 111-162. In French.

Rákosy, L., Goia, M., Kovács, Z. 2003. Catalogul lepidopterelor României [Catalog of the lepidoptera of Romania]. Societatea Lepidopterologică Românâ. ClujNapoca 2003. Bilingual Romanian/German.

Rakosy, L., GoiA, M. 2006. Addenda und Corrigenda zu dem Verzeichnis der Schmetterlinge Rumäniens [Additions and corrections to the list of the Lepidoptera of Romania]. Entomologica romanica, 11:69-79. Bilingual German/Romanian.

Šefrová, H., LAŠTưvKA, Z. 2001. Dispersal of the horsechestnut leaf miner, Cameraria ohridella DeschKa \& Dimić, 1986, in Europe: its course, ways and causes (Lepidoptera: Gracillariidae). Entomologische Zeitschrift. 111:194-198.

SzÉKELY, L. 2014. Istoria lepidopterologiei din România [History of lepidopterology in Romania]. Selfpublished, Braşov. In Romanian
Valeriu ALBU

23032 Oak Meadow Ln., Friant,

CA 93626

E-mail: valalbu@netptc.net
Sebastian Albu

23032 Oak Meadow Ln., Friant,

CA 93626

E-mail: salbu3001@gmail.com

Received: 01.09.2018

Accepted: 31.10 .2018

Published online: 31.12 .2018

Published: 31.12.2018

Online article number: ER22201803

doi: 10.24193/entomolrom.22.3 


\begin{tabular}{|c|c|c|c|c|c|c|c|c|c|c|}
\hline $\begin{array}{c}\mathrm{K} \& \mathrm{R} \\
\text { LIST NR. }\end{array}$ & SUPERFAMILY & FAMILY & GENUS & SPECIES & COUNTY & LOCALITY & YEAR & MONTH & \multicolumn{2}{|c|}{ DAY NR. } \\
\hline 7 & Micropterigoidea & Micropterigidae & Micropterix & aruncella & Braşov & Pădurea Bogata, Hoghiz & 2002 & May & 27 & 3 \\
\hline 7 & Micropterigoidea & Micropterigidae & Micropterix & aruncella & Braşov & Pădurea Bogata, Hoghiz & 2002 & June & 10 & 1 \\
\hline 63 & Hepialoidea & Hepialidae & Triodia & sylvina & Braşov & Braşov, Bartolomeu & 1968 & September & 2 & 1 \\
\hline 63 & Hepialoidea & Hepialidae & Triodia & sylvina & Hunedoara & Sibişel & 2001 & August & 18 & 1 \\
\hline 63 & Hepialoidea & Hepialidae & Triodia & sylvina & Hunedoara & Sibişel & 2002 & July & 11 & 1 \\
\hline 80 & Hepialoidea & Hepialidae & Hepialus & humuli & Braşov & Pietrele lui Solomon, Braşov & 1982 & May & 29 & 1 \\
\hline 346 & Adeloidea & Adelidae & Nemophora & metallica & Braşov & Drăuşeni & 1998 & July & 2 & 1 \\
\hline 352 & Adeloidea & Adelidae & Nemophora & fasciella & Braşov & Drăuşeni & 1991 & July & 16 & 1 \\
\hline 352 & Adeloidea & Adelidae & Nemophora & fasciella & Braşov & Dealul Lempeş, Sânpetru & 2002 & June & 15 & 1 \\
\hline 366 & Adeloidea & Adelidae & Adela & cuprella & Braşov & Drăuşeni & 1998 & July & 2 & 6 \\
\hline 366 & Adeloidea & Adelidae & Adela & cuprella & Braşov & Pădurea Bogata, Hoghiz & 1998 & July & 11 & 3 \\
\hline 366 & Adeloidea & Adelidae & Adela & cuprella & Hunedoara & Sibişel & 2003 & June & 27 & 2 \\
\hline 378 & Adeloidea & Adelidae & Cauchas & leucocerella & Braşov & Pădurea Bogata, Hoghiz & 2007 & May & 27 & 1 \\
\hline 382 & Adeloidea & Adelidae & Cauchas & rufimitrella & Braşov & Dealul Lempeş, Sânpetru & 1982 & May & 29 & 1 \\
\hline 889 & Tineoidea & Psychidae & Bijugis & pectinella & Covasna & Breţcu village & 1982 & July & 23 & 1 \\
\hline 623 & Tineoidea & Tineidae & Nemapogon & granella & Braşov & Braşov, Bartolomeu & 2007 & May & 27 & 1 \\
\hline 624 & Tineoidea & Tineidae & Nemapogon & cloacella & Braşov & Dealul Lempeş, Sânpetru & 2007 & May & 29 & 1 \\
\hline 669 & Tineoidea & Tineidae & Tineola & bisselliella & Braşov & Braşov & 2003 & September & 14 & 1 \\
\hline 689 & Tineoidea & Tineidae & Niditinea & fuscella & Hunedoara & Sibişel & 2003 & July & 2 & 2 \\
\hline 700 & Tineoidea & Tineidae & Monopis & laevigella & Braşov & Braşov & 1980 & June & 8 & 1 \\
\hline 700 & Tineoidea & Tineidae & Monopis & laevigella & Hunedoara & Sibişel & 2003 & July & 1 & 1 \\
\hline 1101 & Gracillarioidea & Gracillariidae & Parectopa & robiniella & Braşov & Şinca Veche & 2017 & October & 14 & 0 \\
\hline 1145 & Gracillarioidea & Gracillariidae & Euspilapteryx & auroguttella & Braşov & Poiana Braşov, 1000 m. & 2007 & May & 22 & 2 \\
\hline 1330 & Gracillarioidea & Gracillariidae & Cameraria & ohridella & Braşov & Braşov & 2017 & October & 1 & 1 \\
\hline 1348 & Yponomeutoidea & Yponomeutidae & Yponomeuta & padella & Covasna & Vâlcele & 2003 & August & 5 & 1 \\
\hline 1354 & Yponomeutoidea & Yponomeutidae & Yponomeuta & plumbella & Hunedoara & Sibişel & 2003 & July & 4 & 1 \\
\hline 1357 & Yponomeutoidea & Yponomeutidae & Euhyponomeuta & stanella & Hunedoara & Sibişel & 2003 & July & 1 & 1 \\
\hline 1453 & Yponomeutoidea & Argyresthiidae & Argyresthia & brockeella & Hunedoara & Sibişel & 2003 & June & 27 & 1 \\
\hline 1458 & Yponomeutoidea & Argyresthiidae & Argyresthia & curvella & Braşov & Tâmpa, Braşov & 2009 & September & 10 & 4 \\
\hline 1458 & Yponomeutoidea & Argyresthiidae & Argyresthia & curvella & Hunedoara & Sibişel & 2003 & July & 10 & 1 \\
\hline
\end{tabular}




\begin{tabular}{|c|c|c|c|c|c|c|c|c|c|c|}
\hline $\begin{array}{c}\text { K \& R } \\
\text { LIST NR. }\end{array}$ & SUPERFAMILY & FAMILY & GENUS & SPECIES & COUNTY & LOCALITY & YEAR & MONTH & \multicolumn{2}{|c|}{ DAY NR. } \\
\hline 1462 & Yponomeutoidea & Argyresthiidae & Argyresthia & spinosella & Braşov & Tâmpa, Braşov & 2002 & June & 17 & 5 \\
\hline 1462 & Yponomeutoidea & Argyresthiidae & Argyresthia & spinosella & Braşov & Dealul Lempeş, Sânpetru & 2002 & June & 17 & 1 \\
\hline 1462 & Yponomeutoidea & Argyresthiidae & Argyresthia & spinosella & Braşov & Dealul Lempeş, Sânpetru & 2007 & May & 29 & 1 \\
\hline 1466 & Yponomeutoidea & Argyresthiidae & Argyresthia & pruniella & Braşov & Dealul Lempeş, Sânpetru & 2002 & June & 17 & 1 \\
\hline 1466 & Yponomeutoidea & Argyresthiidae & Argyresthia & pruniella & Braşov & Dealul Lempeş, Sânpetru & 2003 & August & 5 & 1 \\
\hline 1468 & Yponomeutoidea & Argyresthiidae & Argyresthia & albistria & Braşov & Tâmpa, Braşov & 1998 & July & 14 & 1 \\
\hline 1468 & Yponomeutoidea & Argyresthiidae & Argyresthia & albistria & Braşov & Tâmpa, Braşov & 2009 & September & 10 & 1 \\
\hline 1525 & Yponomeutoidea & Plutellidae & Plutella & xylostella & Braşov & Dealul Lempeş, Sânpetru & 2002 & June & 15 & 2 \\
\hline 1587 & Yponomeutoidea & Glyphipterigidae & Glyphipterix & equitella & Braşov & Tâmpa, Braşov & 2002 & June & 17 & 1 \\
\hline 1592 & Yponomeutoidea & Glyphipterigidae & Glyphipterix & forsterella & Braşov & Pădurea Bogata, Hoghiz & 2007 & May & 27 & 2 \\
\hline 1594 & Yponomeutoidea & Glyphipterigidae & Glyphipterix & simpliciella & Braşov & Valea Gârcinului, Săcele & 1982 & July & 25 & 8 \\
\hline 1594 & Yponomeutoidea & Glyphipterigidae & Glyphipterix & simpliciella & Braşov & Dealul Lempeş, Sânpetru & 2007 & May & 29 & 5 \\
\hline 1482 & Yponomeutoidea & Ypsolophidae & Ypsolopha & dentella & Braşov & Tâmpa, Braşov & 1998 & July & 14 & 1 \\
\hline 1424 & Yponomeutoidea & Praydidae & Prays & fraxinella & Braşov & Dealul Lempeş, Sânpetru & 2002 & June & 15 & 1 \\
\hline 1611 & Yponomeutoidea & Lyonetiidae & Leucoptera & spartifoliella & Braşov & Pădurea Bogata, Hoghiz & 2007 & May & 27 & 1 \\
\hline 2326 & Gelechioidea & Oecophridae & Harpella & forficella & Braşov & Tâmpa, Braşov & 1998 & July & 14 & 1 \\
\hline 2326 & Gelechioidea & Oecophridae & Harpella & forficella & Hunedoara & Sibişel & 2001 & August & 29 & 1 \\
\hline 2328 & Gelechioidea & Oecophridae & Carcina & quercana & Hunedoara & Sibişel & 2002 & July & 15 & 1 \\
\hline 1906 & Gelechioidea & Elachistidae & Elachista & dispunctella & Braşov & Dealul Lempeş, Sânpetru & 2007 & May & 29 & 1 \\
\hline 1997 & Gelechioidea & Elachistidae & Elachista & pollinariella & Braşov & Pădurea Bogata, Hoghiz & 2007 & May & 27 & 1 \\
\hline 2022 & Gelechioidea & Elachistidae & Elachista & subocellea & Braşov & Dealul Lempeş, Sânpetru & 2007 & May & 29 & 6 \\
\hline 1730 & Gelechioidea & Elachistidae & Agonopterix & alstromeriana & Braşov & Braşov & 2003 & September & 14 & 1 \\
\hline 1644 & Gelechioidea & Elachistidae & Ethmia & quadrillella & Braşov & Pădurea Bogata, Hoghiz & 2007 & May & 27 & 1 \\
\hline 1644 & Gelechioidea & Elachistidae & Ethmia & quadrillella & Hunedoara & Sibişel & 1999 & June & 12 & 1 \\
\hline 1644 & Gelechioidea & Elachistidae & Ethmia & quadrillella & Hunedoara & Sibişel & 2000 & August & 12 & 1 \\
\hline 1647 & Gelechioidea & Elachistidae & Ethmia & pusiella & Braşov & Braşov, Bartolomeu & 1969 & August & 6 & 1 \\
\hline 1647 & Gelechioidea & Elachistidae & Ethmia & pusiella & Covasna & Breţcu village & 1982 & July & 23 & 4 \\
\hline 1655 & Gelechioidea & Elachistidae & Ethmia & bipunctella & Covasna & Vâlcele & 2003 & August & 5 & 1 \\
\hline 2456 & Gelechioidea & Coleophoridae & Coleophora & gryphipennella & Braşov & Tâmpa, Braşov & 2002 & June & 17 & 1 \\
\hline 2499 & Gelechioidea & Coleophoridae & Coleophora & frischella & Braşov & Braşov, dealul Warthe & 2007 & May & 29 & 1 \\
\hline 2499 & Gelechioidea & Coleophoridae & Coleophora & frischella & Hunedoara & Sibişel & 2001 & July & 10 & 1 \\
\hline 2499 & Gelechioidea & Coleophoridae & Coleophora & frischella & Hunedoara & Sibişel & 2003 & July & 1 & 4 \\
\hline
\end{tabular}




\begin{tabular}{|c|c|c|c|c|c|c|c|c|c|c|}
\hline $\begin{array}{c}\text { K \& R } \\
\text { LIST NR. }\end{array}$ & SUPERFAMILY & FAMILY & GENUS & SPECIES & COUNTY & LOCALITY & YEAR & MONTH & \multicolumn{2}{|c|}{ DAY NR. } \\
\hline 2583 & Gelechioidea & Coleophoridae & Coleophora & chalcogrammella & Braşov & Tâmpa, Braşov & 1991 & July & 17 & 1 \\
\hline 2587 & Gelechioidea & Coleophoridae & Coleophora & mayrella & Hunedoara & Sibişel & 2003 & July & 1 & 1 \\
\hline 2654 & Gelechioidea & Coleophoridae & Coleophora & lixella & Braşov & Drăuşeni & 1982 & May & 30 & 3 \\
\hline 2079 & Gelechioidea & Scythrdidae & Scythris & seliniella & Braşov & Tâmpa, Braşov & 2002 & May & 17 & 3 \\
\hline 2131 & Gelechioidea & Scythrdidae & Scythris & tributella & Braşov & Pădurea Bogata, Hoghiz & 2007 & May & 27 & 2 \\
\hline 3242 & Gelechioidea & Gelechiidae & Chrysoestia & drurella & Braşov & Dealul Lempeş, Sânpetru & 2007 & May & 29 & 1 \\
\hline 3261 & Gelechioidea & Gelechiidae & Isophrictis & striatella & Braşov & Pădurea Bogata, Hoghiz & 2007 & May & 27 & 3 \\
\hline 3379 & Gelechioidea & Gelechiidae & Bryotropha & galbanella & Braşov & Dealul Lempeș, Sânpetru & 2002 & June & 15 & 1 \\
\hline 3386 & Gelechioidea & Gelechiidae & Bryotropha & similis & Braşov & Dealul Lempeş, Sânpetru & 2007 & May & 29 & 1 \\
\hline 3386 & Gelechioidea & Gelechiidae & Bryotropha & similis & Braşov & Pădurea Bogata, Hoghiz & 2007 & May & 27 & 1 \\
\hline 3410 & Gelechioidea & Gelechiidae & Parachronistis & albiceps & Hunedoara & Sibişel & 1999 & June & 10 & 1 \\
\hline 3472 & Gelechioidea & Gelechiidae & Gelechia & sabinellus & Braşov & Dealul Lempeş, Sânpetru & 2002 & June & 17 & 1 \\
\hline 3477 & Gelechioidea & Gelechiidae & Gelechia & muscosella & Hunedoara & Sibişel & 2003 & June & 29 & 1 \\
\hline 3498 & Gelechioidea & Gelechiidae & Mirificarma & eburnella & Covasna & Breţcu village & 1982 & July & 23 & 2 \\
\hline 3498 & Gelechioidea & Gelechiidae & Mirificarma & eburnella & Braşov & Drăuşeni & 1998 & July & 2 & 1 \\
\hline 3788 & Gelechioidea & Gelechiidae & Syncopacma & cincticulella & Braşov & Pădurea Bogata, Hoghiz & 2007 & May & 27 & 1 \\
\hline 3805 & Gelechioidea & Gelechiidae & Anacampsis & blattariella & Hunedoara & Sibişel & 2002 & July & 28 & 1 \\
\hline 3850 & Gelechioidea & Gelechiidae & Dichomeris & ustalella & Braşov & Pădurea Bogata, Hoghiz & 1998 & July & 11 & 1 \\
\hline 3862 & Gelechioidea & Gelechiidae & Brachmia & dimidiella & Hunedoara & Sibişel & 2003 & July & 6 & 1 \\
\hline 3874 & Gelechioidea & Gelechiidae & Acompsia & cinerella & Braşov & Dealul Lempeş, Sânpetru & 2002 & June & 15 & 1 \\
\hline 3874 & Gelechioidea & Gelechiidae & Acompsia & cinerella & Braşov & Dealul Lempeş, Sânpetru & 2007 & May & 29 & 1 \\
\hline 3874 & Gelechioidea & Gelechiidae & Acompsia & cinerella & Braşov & Pădurea Bogata, Hoghiz & 2007 & May & 27 & 1 \\
\hline 5375 & Pterophoroidea & Pterophoridae & Buszkoiana & capnodactylus & Braşov & Pădurea Bogata, Hoghiz & 2007 & May & 27 & 2 \\
\hline 5390 & Pterophoroidea & Pterophoridae & Stenoptilia & pterodactyla & Braşov & Tâmpa, Braşov & 1982 & June & 12 & 1 \\
\hline 5390 & Pterophoroidea & Pterophoridae & Stenoptilia & pterodactyla & Braşov & Tâmpa, Braşov & 1991 & July & 17 & 1 \\
\hline 5390 & Pterophoroidea & Pterophoridae & Stenoptilia & pterodactyla & Braşov & Dealul Lempeş, Sânpetru & 2002 & June & 17 & 1 \\
\hline 5390 & Pterophoroidea & Pterophoridae & Stenoptilia & pterodactyla & Braşov & Dealul Lempeş, Sânpetru & 2007 & May & 29 & 1 \\
\hline 5390 & Pterophoroidea & Pterophoridae & Stenoptilia & pterodactyla & Braşov & Pădurea Bogata, Hoghiz & 1998 & July & 11 & 1 \\
\hline 5390 & Pterophoroidea & Pterophoridae & Stenoptilia & pterodactyla & Covasna & Vâlcele & 1981 & June & 19 & 1 \\
\hline 5390 & Pterophoroidea & Pterophoridae & Stenoptilia & pterodactyla & Covasna & Breţcu village & 1982 & July & 23 & 1 \\
\hline 5393 & Pterophoroidea & Pterophoridae & Stenoptilia & stigmatodactylus & Braşov & Tâmpa, Braşov & 1991 & July & 17 & 1 \\
\hline 5436 & Pterophoroidea & Pterophoridae & Marasmarcha & lunedactyla & Braşov & Drăuşeni & 1981 & June & 20 & 1 \\
\hline
\end{tabular}




\begin{tabular}{|c|c|c|c|c|c|c|c|c|c|c|}
\hline $\begin{array}{c}\text { K \& R } \\
\text { LIST NR. }\end{array}$ & SUPERFAMILY & FAMILY & GENUS & SPECIES & COUNTY & LOCALITY & YEAR & MONTH & \multicolumn{2}{|c|}{ DAY NR. } \\
\hline 5443 & Pterophoroidea & Pterophoridae & Oxyptilus & parvidactyla & Hunedoara & Sibişel & 2001 & July & 20 & 1 \\
\hline 5445 & Pterophoroidea & Pterophoridae & Oxyptilus & distans & Braşov & Dealul Lempeş, Sânpetru & 2002 & June & 15 & 1 \\
\hline 5485 & Pterophoroidea & Pterophoridae & Pterophorus & pentadactyla & Braşov & Tâmpa, Braşov & 1998 & July & 14 & 1 \\
\hline 5485 & Pterophoroidea & Pterophoridae & Pterophorus & pentadactyla & Hunedoara & Sibişel & 2003 & July & 10 & 1 \\
\hline 5500 & Pterophoroidea & Pterophoridae & Merrifieldia & tridactyla & Braşov & Fundata, $1290 \mathrm{~m}$. & 1998 & July & 13 & 2 \\
\hline 5506 & Pterophoroidea & Pterophoridae & Merrifieldia & baliodactylus & Braşov & Tâmpa, Braşov & 1991 & July & 17 & 2 \\
\hline 5513 & Pterophoroidea & Pterophoridae & Wheeleria & obsoletus & Braşov & Pădurea Bogata, Hoghiz & 1998 & July & 11 & 1 \\
\hline 5521 & Pterophoroidea & Pterophoridae & Pselnophorus & heterodactyla & Braşov & Pădurea Bogata, Hoghiz & 1998 & July & 11 & 1 \\
\hline 5534 & Pterophoroidea & Pterophoridae & Hellinsia & tephradactyla & Braşov & Pădurea Bogata, Hoghiz & 1998 & July & 11 & 1 \\
\hline 5552 & Pterophoroidea & Pterophoridae & Emmelina & monodactyla & Braşov & Dealul Lempeş, Sânpetru & 2007 & May & 29 & 1 \\
\hline 5552 & Pterophoroidea & Pterophoridae & Emmelina & monodactyla & Hunedoara & Sibişel & 2001 & July & 10 & 1 \\
\hline 5269 & Choreutoidea & Choreutidae & Anthophila & fabriciana & Braşov & Dealul Bunloc, Săcele & 1981 & July & 26 & 1 \\
\hline 5269 & Choreutoidea & Choreutidae & Anthophila & fabriciana & Braşov & Poiana Braşov, 1000 m. & 1981 & July & 26 & 1 \\
\hline 5269 & Choreutoidea & Choreutidae & Anthophila & fabriciana & Braşov & Dealul Lempeş, Sânpetru & 2007 & May & 27 & 1 \\
\hline 5269 & Choreutoidea & Choreutidae & Anthophila & fabriciana & Braşov & Pădurea Bogata, Hoghiz & 2002 & June & 10 & 2 \\
\hline 5269 & Choreutoidea & Choreutidae & Anthophila & fabriciana & Braşov & Pădurea Bogata, Hoghiz & 2007 & May & 27 & 1 \\
\hline 5269 & Choreutoidea & Choreutidae & Anthophila & fabriciana & Covasna & Vâlcele & 1981 & June & 19 & 1 \\
\hline 4271 & Tortricoidea & Tortricidae & Agapeta & zoegana & Covasna & Vâlcele & 2003 & August & 5 & 1 \\
\hline 4277 & Tortricoidea & Tortricidae & Fulvoclysia & nerminae & Hunedoara & Sibişel & 2002 & July & 13 & 1 \\
\hline 4277 & Tortricoidea & Tortricidae & Fulvoclysia & nerminae & Hunedoara & Sibişel & 2002 & June & 27 & 1 \\
\hline 4294 & Tortricoidea & Tortricidae & Aethes & hartmanniana & Braşov & Dealul Lempeş, Sânpetru & 2002 & June & 15 & 3 \\
\hline 4326 & Tortricoidea & Tortricidae & Aethes & cnicana & Braşov & Dealul Lempeş, Sânpetru & 2007 & May & 29 & 3 \\
\hline 4411 & Tortricoidea & Tortricidae & Acleris & lipsiana & Braşov & Braşov & 2003 & September & 14 & 1 \\
\hline 4420 & Tortricoidea & Tortricidae & Neosphaleroptera & nubilana & Braşov & Tâmpa, Braşov & 2002 & June & 17 & 1 \\
\hline 4420 & Tortricoidea & Tortricidae & Neosphaleroptera & nubilana & Braşov & Dealul Lempeş, Sânpetru & 2002 & June & 15 & 3 \\
\hline 4443 & Tortricoidea & Tortricidae & Eana & osseana & Braşov & Fundata, $1290 \mathrm{~m}$. & 1998 & July & 13 & 1 \\
\hline 4444 & Tortricoidea & Tortricidae & Eana & argentana & Braşov & Tâmpa, Braşov & 1980 & June & 21 & 1 \\
\hline 4444 & Tortricoidea & Tortricidae & Eana & argentana & Braşov & Drăuşeni & 1998 & July & 2 & 1 \\
\hline 4471 & Tortricoidea & Tortricidae & Cnephasia & incertana & Braşov & Dealul Lempeş, Sânpetru & 2002 & June & 15 & 1 \\
\hline 4520 & Tortricoidea & Tortricidae & Eulia & ministrana & Braşov & Dealul Lempeş, Sânpetru & 1981 & May & 31 & 1 \\
\hline 4557 & Tortricoidea & Tortricidae & Archips & podana & Hunedoara & Sibişel & 1999 & August & 10 & 1 \\
\hline 4557 & Tortricoidea & Tortricidae & Archips & podana & Hunedoara & Sibişel & 2001 & August & 21 & 1 \\
\hline
\end{tabular}




\begin{tabular}{|c|c|c|c|c|c|c|c|c|c|c|}
\hline $\begin{array}{c}\text { K \& R } \\
\text { LIST NR. }\end{array}$ & SUPERFAMILY & FAMILY & GENUS & SPECIES & COUNTY & LOCALITY & YEAR & MONTH & DAY & NR. \\
\hline 4557 & Tortricoidea & Tortricidae & Archips & podana & Hunedoara & Sibişel & 2003 & June & 27 & 1 \\
\hline 4557 & Tortricoidea & Tortricidae & Archips & podana & Hunedoara & Sibişel & 2003 & July & 1 & 1 \\
\hline 4559 & Tortricoidea & Tortricidae & Archips & xylosteana & Braşov & Pădurea Bogata, Hoghiz & 1998 & July & 11 & 1 \\
\hline 4559 & Tortricoidea & Tortricidae & Archips & xylosteana & Hunedoara & Sibişel & 2003 & June & 23 & 1 \\
\hline 4559 & Tortricoidea & Tortricidae & Archips & xylosteana & Hunedoara & Sibişel & 2003 & June & 29 & 1 \\
\hline 4559 & Tortricoidea & Tortricidae & Archips & xylosteana & Hunedoara & Sibişel & 2003 & July & 1 & 1 \\
\hline 4581 & Tortricoidea & Tortricidae & Pandemis & dumetana & Hunedoara & Sibişel & 2002 & July & 19 & 1 \\
\hline 4616 & Tortricoidea & Tortricidae & Clepsis & senecionana & Hunedoara & Sibişel & 1999 & June & 10 & 1 \\
\hline 4618 & Tortricoidea & Tortricidae & Clepsis & rurinana & Braşov & Tâmpa, Braşov & 2002 & June & 17 & 1 \\
\hline 4644 & Tortricoidea & Tortricidae & Isotrias & hybridana & Braşov & Tâmpa, Braşov & 2002 & June & 17 & 2 \\
\hline 4644 & Tortricoidea & Tortricidae & Isotrias & hybridana & Braşov & Dealul Lempeş, Sânpetru & 2002 & June & 17 & 1 \\
\hline 4667 & Tortricoidea & Tortricidae & Endothenia & marginana & Braşov & Dealul Lempeş, Sânpetru & 2007 & May & 29 & 1 \\
\hline 4669 & Tortricoidea & Tortricidae & Endothenia & ustulana & Braşov & Dealul Lempeş, Sânpetru & 2007 & May & 29 & 1 \\
\hline 4671 & Tortricoidea & Tortricidae & Endothenia & nigricostana & Hunedoara & Sibişel & 2003 & July & 2 & 1 \\
\hline 4698 & Tortricoidea & Tortricidae & Apotomis & inundana & Hunedoara & Sibişel & 2000 & August & 7 & 1 \\
\hline 4698 & Tortricoidea & Tortricidae & Apotomis & inundana & Hunedoara & Sibişel & 2003 & July & 1 & 1 \\
\hline 4706 & Tortricoidea & Tortricidae & Apotomis & sororculana & Hunedoara & Sibişel & 2003 & June & 29 & 1 \\
\hline 4713 & Tortricoidea & Tortricidae & Hedya & salicella & Covasna & Brețcu village & 1982 & July & 23 & 2 \\
\hline 4713 & Tortricoidea & Tortricidae & Hedya & salicella & Hunedoara & Sibişel & 1999 & July & 19 & 1 \\
\hline 4713 & Tortricoidea & Tortricidae & Hedya & salicella & Hunedoara & Sibişel & 1999 & August & 14 & 1 \\
\hline 4713 & Tortricoidea & Tortricidae & Hedya & salicella & Hunedoara & Sibişel & 2001 & August & 20 & 1 \\
\hline 4713 & Tortricoidea & Tortricidae & Hedya & salicella & Hunedoara & Sibişel & 2001 & August & 25 & 1 \\
\hline 4713 & Tortricoidea & Tortricidae & Hedya & salicella & Hunedoara & Sibişel & 2003 & June & 23 & 1 \\
\hline 4714 & Tortricoidea & Tortricidae & Hedya & nubiferana & Braşov & Pădurea Bogata, Hoghiz & 2007 & May & 27 & 1 \\
\hline 4714 & Tortricoidea & Tortricidae & Hedya & nubiferana & Braşov & Dealul Lempeş, Sânpetru & 2007 & May & 29 & 2 \\
\hline 4714 & Tortricoidea & Tortricidae & Hedya & nubiferana & Hunedoara & Sibişel & 2003 & June & 23 & 1 \\
\hline 4714 & Tortricoidea & Tortricidae & Hedya & nubiferana & Hunedoara & Sibişel & 2003 & June & 29 & 1 \\
\hline 4722 & Tortricoidea & Tortricidae & Celypha & striana & Braşov & Tâmpa, Braşov & 1982 & June & 11 & 1 \\
\hline 4722 & Tortricoidea & Tortricidae & Celypha & striana & Braşov & Pădurea Bogata, Hoghiz & 1998 & July & 11 & 2 \\
\hline 4728 & Tortricoidea & Tortricidae & Celypha & cespitana & Hunedoara & Sibişel & 2001 & August & 24 & 1 \\
\hline 4731 & Tortricoidea & Tortricidae & Celypha & lacunana & Braşov & Dealul Lempeş, Sânpetru & 2002 & June & 15 & 2 \\
\hline 4731 & Tortricoidea & Tortricidae & Celypha & lacunana & Braşov & Dealul Lempeş, Sânpetru & 2007 & May & 29 & 4 \\
\hline
\end{tabular}




\begin{tabular}{|c|c|c|c|c|c|c|c|c|c|c|}
\hline $\begin{array}{c}\text { K \& R } \\
\text { LIST NR. }\end{array}$ & SUPERFAMILY & FAMILY & GENUS & SPECIES & COUNTY & LOCALITY & YEAR & MONTH & \multicolumn{2}{|c|}{ DAY NR } \\
\hline 4731 & Tortricoidea & Tortricidae & Celypha & lacunana & Braşov & Tâmpa, Braşov & 2017 & August & 29 & 1 \\
\hline 4733 & Tortricoidea & Tortricidae & Celypha & rivulana & Hunedoara & Sibişel & 2003 & July & 10 & 1 \\
\hline 4740 & Tortricoidea & Tortricidae & Phiaris & umbrosana & Braşov & Pădurea Bogata, Hoghiz & 2002 & June & 10 & 1 \\
\hline 4750 & Tortricoidea & Tortricidae & Phiaris & palustrana & Braşov & Tâmpa, Braşov & 2002 & June & 17 & 5 \\
\hline 4817 & Tortricoidea & Tortricidae & Thiodia & citrana & Braşov & Dealul Lempeş, Sânpetru & 2002 & June & 15 & 4 \\
\hline 4840 & Tortricoidea & Tortricidae & Epinotia & trigonella & Hunedoara & Sibişel & 2003 & July & 10 & 1 \\
\hline 4844 & Tortricoidea & Tortricidae & Epinotia & solandriana & Braşov & Dealul Bunloc, Săcele & 1981 & July & 26 & 1 \\
\hline 4845 & Tortricoidea & Tortricidae & Epinotia & abbreviana & Braşov & Tâmpa, Braşov & 2002 & June & 17 & 1 \\
\hline 4845 & Tortricoidea & Tortricidae & Epinotia & abbreviana & Hunedoara & Sibişel & 2003 & Jun & 29 & 1 \\
\hline 4850 & Tortricoidea & Tortricidae & Epinotia & cruciana & Braşov & Hoghiz & 1998 & July & 11 & 1 \\
\hline 4859 & Tortricoidea & Tortricidae & Epinotia & huebneriana & Covasna & Vâlcele & 2003 & August & 5 & 1 \\
\hline 4866 & Tortricoidea & Tortricidae & Epinotia & pygmaeana & Braşov & Şapte Scări, muntele Piatra Mare & 2004 & April & 21 & 4 \\
\hline 4896 & Tortricoidea & Tortricidae & Pelochrista & caecimaculana & Braşov & Dealul Lempeş, Sânpetru & 2002 & June & 17 & 1 \\
\hline 4949 & Tortricoidea & Tortricidae & Eucosma & lacteana & Braşov & Dealul Lempeş, Sânpetru & 2007 & May & 29 & 1 \\
\hline 4985 & Tortricoidea & Tortricidae & Gypsonoma & dealbana & Hunedoara & Sibişel & 2003 & June & 27 & 1 \\
\hline 4993 & Tortricoidea & Tortricidae & Epiblema & sticticana & Hunedoara & Sibişel & 1999 & August & 11 & 1 \\
\hline 4994 & Tortricoidea & Tortricidae & Epiblema & scutulana & Braşov & Dealul Lempeş, Sânpetru & 2002 & June & 15 & 1 \\
\hline 5021 & Tortricoidea & Tortricidae & Notocelia & uddmanniana & Hunedoara & Sibişel & 2003 & June & 29 & 1 \\
\hline 5021 & Tortricoidea & Tortricidae & Notocelia & uddmanniana & Hunedoara & Sibişel & 2003 & July & 1 & 1 \\
\hline 5022 & Tortricoidea & Tortricidae & Notocelia & roborana & Hunedoara & Sibişel & 2003 & June & 27 & 1 \\
\hline 5025 & Tortricoidea & Tortricidae & Notocelia & rosaecolana & Braşov & Roadeş & 2002 & June & 18 & 1 \\
\hline 5084 & Tortricoidea & Tortricidae & Cydia & compositella & Braşov & Dealul Lempeş, Sânpetru & 2007 & May & 29 & 1 \\
\hline 5093 & Tortricoidea & Tortricidae & Cydia & caecana & Braşov & Dealul Lempeş, Sânpetru & 2002 & June & 15 & 5 \\
\hline 5093 & Tortricoidea & Tortricidae & Cydia & caecana & Braşov & Dealul Lempeş, Sânpetru & 2007 & May & 29 & 1 \\
\hline 5139 & Tortricoidea & Tortricidae & Cydia & strobilella & Braşov & Poiana Braşov & 1982 & May & 29 & 1 \\
\hline 5144 & Tortricoidea & Tortricidae & Cydia & pomonella & Hunedoara & Sibişel & 2001 & September & 5 & 1 \\
\hline 5153 & Tortricoidea & Tortricidae & Cydia & fagiglandana & Hunedoara & Sibişel & 2003 & June & 22 & 1 \\
\hline 5163 & Tortricoidea & Tortricidae & Lathronympha & strigana & Hunedoara & Sibişel & 2001 & August & 21 & 1 \\
\hline 5170 & Tortricoidea & Tortricidae & Pammene & amygdalana & Braşov & Poiana Braşov, $1000 \mathrm{~m}$. & 2007 & May & 22 & 1 \\
\hline 5187 & Tortricoidea & Tortricidae & Pammene & agnotana & Braşov & Hoghiz & 1998 & July & 11 & 1 \\
\hline 5205 & Tortricoidea & Tortricidae & Pammene & germmana & Braşov & Dealul Lempeş, Sânpetru & 2007 & May & 29 & 1 \\
\hline 5214 & Tortricoidea & Tortricidae & Dichrorampha & plumbana & Braşov & Poiana Braşov, $1000 \mathrm{~m}$. & 2007 & May & 22 & 2 \\
\hline
\end{tabular}




\begin{tabular}{|c|c|c|c|c|c|c|c|c|c|c|}
\hline $\begin{array}{c}\text { K \& R } \\
\text { LIST NR. }\end{array}$ & SUPERFAMILY & FAMILY & GENUS & SPECIES & COUNTY & LOCALITY & YEAR & MONTH & DAY & NR. \\
\hline 5214 & Tortricoidea & Tortricidae & Dichrorampha & plumbana & Braşov & Pădurea Bogata, Hoghiz & 2007 & May & 27 & 4 \\
\hline 5222 & Tortricoidea & Tortricidae & Dichrorampha & eximia & Braşov & Dealul Lempeş, Sânpetru & 2007 & May & 29 & 2 \\
\hline 5240 & Tortricoidea & Tortricidae & Dichrorampha & sequana & Braşov & Dealul Lempeş, Sânpetru & 2007 & May & 29 & 3 \\
\hline 5246 & Tortricoidea & Tortricidae & Dichrorampha & vancouverana & Braşov & Dealul Lempeş, Sânpetru & 2002 & June & 15 & 2 \\
\hline 5248 & Tortricoidea & Tortricidae & Dichrorampha & alpinana & Braşov & Dealul Lempeş, Sânpetru & 2002 & June & 15 & 2 \\
\hline 5249 & Tortricoidea & Tortricidae & Dichrorampha & petiverella & Braşov & Dealul Lempeş, Sânpetru & 2002 & June & 15 & 2 \\
\hline 5249 & Tortricoidea & Tortricidae & Dichrorampha & petiverella & Braşov & Dealul Lempeş, Sânpetru & 2007 & May & 29 & 6 \\
\hline 4151 & Cossoidea & Cossidae & Cossus & cossus & Covasna & Breţcu village & 1982 & July & 23 & 2 \\
\hline 4176 & Cossoidea & Cossidae & Zeuzera & pyrina & Braşov & Braşov & 1969 & August & 2 & 1 \\
\hline 4026 & Cossoidea & Sesiidae & Pennisetia & hylaeiformis & Braşov & Drăuşeni & 1969 & August & 4 & 1 \\
\hline 4039 & Cossoidea & Sesiidae & Paranthrene & tabaniformis & Braşov & Pădurea Bogata, Hoghiz & 1998 & July & 2 & 1 \\
\hline 4060 & Cossoidea & Sesiidae & Synanthedon & myopaeformis & Braşov & Drăuşeni & 1966 & June & 2 & 1 \\
\hline 4064 & Cossoidea & Sesiidae & Synanthedon & tipuliformis & Braşov & Drăuşeni & 1966 & June & 3 & 1 \\
\hline 4064 & Cossoidea & Sesiidae & Synanthedon & tipuliformis & Braşov & Braşov, Bartolomeu & 1969 & July & 27 & 1 \\
\hline 4130 & Cossoidea & Sesiidae & Chamaesphecia & bibioniformis & Braşov & Braşov, dealul Warthe & 1968 & July & 2 & 1 \\
\hline 4139 & Cossoidea & Sesiidae & Chamaesphecia & tenthrediniformis & Braşov & Dealul Lempeş, Sânpetru & 2002 & June & 15 & 1 \\
\hline 3907 & Zygaenoidea & Limacodidae & Apoda & limacodes & Hunedoara & Sibişel & 1999 & June & 10 & 1 \\
\hline 3907 & Zygaenoidea & Limacodidae & Apoda & limacodes & Hunedoara & Sibişel & 2003 & June & 28 & 1 \\
\hline 3938 & Zygaenoidea & Zygaenidae & Jordanita & subsolana & Braşov & Teliu, 1500 m., munţii Întorsurii & 1978 & July & 2 & 1 \\
\hline 3941 & Zygaenoidea & Zygaenidae & Jordanita & chloros & Braşov & Tâmpa, Braşov & 1981 & July & 25 & 1 \\
\hline 3941 & Zygaenoidea & Zygaenidae & Jordanita & chloros & Braşov & Drăuşeni & 1998 & July & 2 & 1 \\
\hline 3943 & Zygaenoidea & Zygaenidae & Jordanita & globulariae & Covasna & Vâlcele & 1981 & June & 19 & 1 \\
\hline 3943 & Zygaenoidea & Zygaenidae & Jordanita & globulariae & Braşov & Vlădeni & 1978 & July & 1 & 1 \\
\hline 3956 & Zygaenoidea & Zygaenidae & Adscita & statices & Braşov & Vlădeni & 1978 & July & 1 & 1 \\
\hline 3974 & Zygaenoidea & Zygaenidae & Zygaena & purpuralis & Braşov & Braşov, Bartolomeu & 1968 & July & 2 & 1 \\
\hline 3974 & Zygaenoidea & Zygaenidae & Zygaena & purpuralis & Braşov & Tâmpa, Braşov & 1969 & July & 18 & 1 \\
\hline 3974 & Zygaenoidea & Zygaenidae & Zygaena & purpuralis & Braşov & Tâmpa, Braşov & 1991 & July & 17 & 2 \\
\hline 3974 & Zygaenoidea & Zygaenidae & Zygaena & purpuralis & Braşov & Fundata, $1290 \mathrm{~m}$. & 1982 & July & 19 & 1 \\
\hline 3974 & Zygaenoidea & Zygaenidae & Zygaena & purpuralis & Braşov & Fundata, $1290 \mathrm{~m}$. & 1998 & July & 13 & 13 \\
\hline 3974 & Zygaenoidea & Zygaenidae & Zygaena & purpuralis & Braşov & Dealul Bunloc, Săcele & 1969 & July & 18 & 1 \\
\hline 3974 & Zygaenoidea & Zygaenidae & Zygaena & purpuralis & Covasna & Brețcu village & 1982 & July & 24 & 1 \\
\hline 3983 & Zygaenoidea & Zygaenidae & Zygaena & loti & Braşov & Braşov, Bartolomeu & 1967 & July & 30 & 1 \\
\hline
\end{tabular}




\begin{tabular}{|c|c|c|c|c|c|c|c|c|c|c|}
\hline $\begin{array}{c}\mathrm{K} \& \mathrm{R} \\
\text { LIST NR. }\end{array}$ & SUPERFAMILY & FAMILY & GENUS & SPECIES & COUNTY & LOCALITY & YEAR & MONTH & \multicolumn{2}{|c|}{ DAY NR } \\
\hline 3983 & Zygaenoidea & Zygaenidae & Zygaena & loti & Braşov & Dealul Lempeş, Sânpetru & 1981 & July & 23 & 1 \\
\hline 3983 & Zygaenoidea & Zygaenidae & Zygaena & loti & Braşov & Tâmpa, Braşov & 1981 & July & 25 & 1 \\
\hline 3983 & Zygaenoidea & Zygaenidae & Zygaena & loti & Braşov & Tâmpa, Braşov & 1981 & July & 30 & 1 \\
\hline 3983 & Zygaenoidea & Zygaenidae & Zygaena & loti & Braşov & Tâmpa, Braşov & 1991 & July & 17 & 2 \\
\hline 3983 & Zygaenoidea & Zygaenidae & Zygaena & loti & Braşov & Tâmpa, Braşov & 1998 & July & 14 & 2 \\
\hline 3983 & Zygaenoidea & Zygaenidae & Zygaena & loti & Braşov & Fundata, $1290 \mathrm{~m}$. & 1998 & July & 13 & 1 \\
\hline 3983 & Zygaenoidea & Zygaenidae & Zygaena & loti & Braşov & Drăuşeni & 1981 & June & 20 & 1 \\
\hline 3992 & Zygaenoidea & Zygaenidae & Zygaena & viciae & Braşov & Fundata, $1290 \mathrm{~m}$. & 1998 & July & 13 & 2 \\
\hline 3992 & Zygaenoidea & Zygaenidae & Zygaena & viciae & Braşov & Tâmpa, Braşov & 1998 & July & 14 & 1 \\
\hline 3995 & Zygaenoidea & Zygaenidae & Zygaena & ephialtes & Braşov & Tâmpa, Braşov & 1998 & July & 14 & 1 \\
\hline 3997 & Zygaenoidea & Zygaenidae & Zygaena & angelicae & Covasna & Breţcu village & 1982 & July & 23 & 4 \\
\hline 3998 & Zygaenoidea & Zygaenidae & Zygaena & filipendulae & Braşov & Drăuşeni & 1981 & June & 20 & 2 \\
\hline 3998 & Zygaenoidea & Zygaenidae & Zygaena & filipendulae & Braşov & Drăuşeni & 1998 & July & 2 & 2 \\
\hline 3998 & Zygaenoidea & Zygaenidae & Zygaena & filipendulae & Braşov & Pădurea Bogata, Hoghiz & 1998 & July & 11 & 1 \\
\hline 3998 & Zygaenoidea & Zygaenidae & Zygaena & filipendulae & Braşov & Valea Gârcinului, Săcele & 1982 & July & 25 & 1 \\
\hline 3998 & Zygaenoidea & Zygaenidae & Zygaena & filipendulae & Braşov & Fundata, 1290 m. & 1998 & July & 13 & 9 \\
\hline 3998 & Zygaenoidea & Zygaenidae & Zygaena & filipendulae & Braşov & Poiana Braşov, 1000 m. & 1982 & July & 18 & 1 \\
\hline 3998 & Zygaenoidea & Zygaenidae & Zygaena & filipendulae & Braşov & Poiana Braşov, $1000 \mathrm{~m}$. & 1991 & July & 17 & 1 \\
\hline 3998 & Zygaenoidea & Zygaenidae & Zygaena & filipendulae & Covasna & Breţcu village & 1982 & July & 23 & 6 \\
\hline 5562 & Thyridoidea & Thyrididae & Thyris & fenestrella & Braşov & Braşov, Bartolomeu & 1968 & June & 28 & 1 \\
\hline 6953 & Papilionoidea & Papilionidae & Parnassius & mnemosyne & Braşov & Poiana Braşov, $1000 \mathrm{~m}$. & 1967 & July & 29 & 1 \\
\hline 6953 & Papilionoidea & Papilionidae & Parnassius & mnemosyne & Braşov & Poiana Braşov, 1000 m. & 1968 & May & 26 & 1 \\
\hline 6953 & Papilionoidea & Papilionidae & Parnassius & mnemosyne & Braşov & Muntele Postăvar, 2000 m. & 1968 & June & 23 & 1 \\
\hline 6953 & Papilionoidea & Papilionidae & Parnassius & mnemosyne & Braşov & Cheile Râşnoavei, Râşnov & 1981 & June & 22 & 1 \\
\hline 6958 & Papilionoidea & Papilionidae & Iphiclides & podalirius & Braşov & Braşov, Bartolomeu & 1967 & May & 4 & 1 \\
\hline 6958 & Papilionoidea & Papilionidae & Iphiclides & podalirius & Braşov & Tâmpa, Braşov & 1981 & April & 22 & 1 \\
\hline 6958 & Papilionoidea & Papilionidae & Iphiclides & podalirius & Hunedoara & Costeşti & 1991 & July & 8 & 1 \\
\hline 6960 & Papilionoidea & Papilionidae & Papilio & machaon & Braşov & Tâmpa, Braşov & 1967 & May & 14 & 1 \\
\hline 6960 & Papilionoidea & Papilionidae & Papilio & machaon & Braşov & Tâmpa, Braşov & 1968 & April & 23 & 1 \\
\hline 6960 & Papilionoidea & Papilionidae & Papilio & machaon & Braşov & Tâmpa, Braşov & 1968 & June & 25 & 1 \\
\hline 6960 & Papilionoidea & Papilionidae & Papilio & machaon & Braşov & Dealul Lempeş, Sânpetru & 1979 & April & 30 & 1 \\
\hline 6960 & Papilionoidea & Papilionidae & Papilio & machaon & Braşov & Dealul Lempeş, Sânpetru & 1982 & May & 29 & 1 \\
\hline
\end{tabular}




\begin{tabular}{|c|c|c|c|c|c|c|c|c|c|c|}
\hline $\begin{array}{c}\text { K \& R } \\
\text { LIST NR. }\end{array}$ & SUPERFAMILY & FAMILY & GENUS & SPECIES & COUNTY & LOCALITY & YEAR & MONTH & \multicolumn{2}{|c|}{ DAY NR. } \\
\hline 6960 & Papilionoidea & Papilionidae & Papilio & machaon & Braşov & Săcele & 1982 & July & 25 & 1 \\
\hline 6879 & Papilionoidea & Hesperiidae & Erynnis & tages & Braşov & Dealul Lempeş, Sânpetru & 2003 & August & 5 & 1 \\
\hline 6882 & Papilionoidea & Hesperiidae & Carcharodus & alceae & Braşov & Drăuşeni & 1966 & August & 30 & 1 \\
\hline 6899 & Papilionoidea & Hesperiidae & Pyrgus & carthami & Braşov & Braşov, Bartolomeu & 1968 & May & 30 & 1 \\
\hline 6899 & Papilionoidea & Hesperiidae & Pyrgus & carthami & Braşov & Dealul Lempeş, Sânpetru & 1981 & May & 31 & 1 \\
\hline 6899 & Papilionoidea & Hesperiidae & Pyrgus & carthami & Braşov & Dealul Lempeş, Sânpetru & 1982 & May & 25 & 1 \\
\hline 6899 & Papilionoidea & Hesperiidae & Pyrgus & carthami & Braşov & Dealul Lempeş, Sânpetru & 1982 & May & 29 & 1 \\
\hline 6899 & Papilionoidea & Hesperiidae & Pyrgus & carthami & Braşov & Tâmpa, Braşov & 1982 & June & 12 & 1 \\
\hline 6904 & Papilionoidea & Hesperiidae & Pyrgus & malvae & Braşov & Braşov, Bartolomeu & 1966 & August & 10 & 1 \\
\hline 6904 & Papilionoidea & Hesperiidae & Pyrgus & malvae & Braşov & Dealul Lempeş, Sânpetru & 2003 & August & 5 & 1 \\
\hline 6904 & Papilionoidea & Hesperiidae & Pyrgus & malvae & Braşov & Pădurea Bogata, Hoghiz & 1967 & July & 31 & 1 \\
\hline 6904 & Papilionoidea & Hesperiidae & Pyrgus & malvae & Braşov & Pădurea Bogata, Hoghiz & 1977 & July & 18 & 1 \\
\hline 6912 & Papilionoidea & Hesperiidae & Pyrgus & alveus & Braşov & Braşov, Bartolomeu & 1966 & August & 6 & 1 \\
\hline 6912 & Papilionoidea & Hesperiidae & Pyrgus & alveus & Braşov & Braşov, Bartolomeu & 1966 & August & 15 & 1 \\
\hline 6019 & Papilionoidea & Hesperiidae & Carterocephalus & palaemon & Braşov & Poiana Braşov, 1000 m. & 1968 & May & 5 & 2 \\
\hline 6019 & Papilionoidea & Hesperiidae & Carterocephalus & palaemon & Braşov & Pădurea Bogata, Hoghiz & 1979 & May & 27 & 1 \\
\hline 6019 & Papilionoidea & Hesperiidae & Carterocephalus & palaemon & Braşov & Şapte Scări, muntele Piatra Mare & 1982 & May & 31 & 1 \\
\hline 6019 & Papilionoidea & Hesperiidae & Carterocephalus & palaemon & Braşov & Dealul Lempeş, Sânpetru & 1981 & May & 31 & 2 \\
\hline 6019 & Papilionoidea & Hesperiidae & Carterocephalus & palaemon & Braşov & Dealul Lempeş, Sânpetru & 1982 & May & 29 & 1 \\
\hline 6923 & Papilionoidea & Hesperiidae & Thymelicus & lineola & Braşov & Braşov, Bartolomeu & 1966 & July & 14 & 1 \\
\hline 6923 & Papilionoidea & Hesperiidae & Thymelicus & lineola & Braşov & Braşov, Bartolomeu & 1967 & July & 14 & 1 \\
\hline 6923 & Papilionoidea & Hesperiidae & Thymelicus & lineola & Braşov & Braşov, dealul Warthe & 1969 & July & 24 & 1 \\
\hline 6923 & Papilionoidea & Hesperiidae & Thymelicus & lineola & Braşov & Dealul Lempeş, Sânpetru & 1979 & July & 14 & 2 \\
\hline 6923 & Papilionoidea & Hesperiidae & Thymelicus & lineola & Braşov & Dealul Lempeş, Sânpetru & 1981 & July & 23 & 1 \\
\hline 6923 & Papilionoidea & Hesperiidae & Thymelicus & lineola & Braşov & Tâmpa, Braşov & 1981 & July & 25 & 1 \\
\hline 6923 & Papilionoidea & Hesperiidae & Thymelicus & lineola & Covasna & Breţcu village & 1982 & July & 23 & 1 \\
\hline 6924 & Papilionoidea & Hesperiidae & Thymelicus & sylvestris & Braşov & Braşov, Bartolomeu & 1969 & August & 7 & 2 \\
\hline 6924 & Papilionoidea & Hesperiidae & Thymelicus & sylvestris & Covasna & Brețcu village & 1982 & July & 23 & 3 \\
\hline 6925 & Papilionoidea & Hesperiidae & Thymelicus & acteon & Braşov & Dealul Lempeş, Sânpetru & 1981 & July & 23 & 1 \\
\hline 6928 & Papilionoidea & Hesperiidae & Hesperia & comma & Braşov & Braşov, Bartolomeu & 1969 & August & 22 & 1 \\
\hline 6928 & Papilionoidea & Hesperiidae & Hesperia & comma & Braşov & Drăuşeni & 1969 & August & 17 & 1 \\
\hline 6930 & Papilionoidea & Hesperiidae & Ochlodes & sylvanus & Braşov & Braşov, Bartolomeu & 1968 & June & 5 & 1 \\
\hline
\end{tabular}




\begin{tabular}{|c|c|c|c|c|c|c|c|c|c|c|}
\hline $\begin{array}{c}\text { K \& R } \\
\text { LIST NR. }\end{array}$ & SUPERFAMILY & FAMILY & GENUS & SPECIES & COUNTY & LOCALITY & YEAR & MONTH & \multicolumn{2}{|c|}{ DAY NR } \\
\hline 6930 & Papilionoidea & Hesperiidae & Ochlodes & sylvanus & Braşov & Braşov, Bartolomeu & 1968 & June & 25 & 1 \\
\hline 6930 & Papilionoidea & Hesperiidae & Ochlodes & sylvanus & Braşov & Braşov, Bartolomeu & 1969 & July & 18 & 2 \\
\hline 6930 & Papilionoidea & Hesperiidae & Ochlodes & sylvanus & Braşov & Braşov, dealul Warthe & 1968 & June & 19 & 1 \\
\hline 6930 & Papilionoidea & Hesperiidae & Ochlodes & sylvanus & Braşov & Tâmpa, Braşov & 1968 & July & 1 & 1 \\
\hline 6930 & Papilionoidea & Hesperiidae & Ochlodes & sylvanus & Braşov & Dealul Bunloc, Săcele & 1991 & July & 15 & 1 \\
\hline 6930 & Papilionoidea & Hesperiidae & Ochlodes & sylvanus & Braşov & Drăuşeni & 1991 & July & 16 & 1 \\
\hline 6966 & Papilionoidea & Pieridae & Leptidea & sinapis & Braşov & Poiana Braşov, $1000 \mathrm{~m}$. & 1966 & May & 25 & 1 \\
\hline 6966 & Papilionoidea & Pieridae & Leptidea & sinapis & Braşov & Drăuşeni & 1982 & May & 30 & 1 \\
\hline 6973 & Papilionoidea & Pieridae & Anthocharis & cardamines & Braşov & Dealul Lempeş, Sânpetru & 1979 & April & 30 & 1 \\
\hline 6993 & Papilionoidea & Pieridae & Aporia & crataegi & Braşov & Poiana Braşov, 1000 m. & 1968 & June & 19 & 1 \\
\hline 6993 & Papilionoidea & Pieridae & Aporia & crataegi & Braşov & Tâmpa, Braşov & 1982 & June & 12 & 1 \\
\hline 6995 & Papilionoidea & Pieridae & Pieris & brassicae & Braşov & Dealul Lempeş, Sânpetru & 1982 & May & 29 & 3 \\
\hline 6998 & Papilionoidea & Pieridae & Pieris & rapae & Braşov & Dealul Bunloc, Săcele & 1979 & May & 29 & 1 \\
\hline 7000 & Papilionoidea & Pieridae & Pieris & napi & Braşov & Dealul Lempeş, Sânpetru & 1979 & April & 30 & 1 \\
\hline 7000 & Papilionoidea & Pieridae & Pieris & napi & Braşov & Muntele Postăvar, 2000 m. & 1968 & June & 17 & 1 \\
\hline 7000 & Papilionoidea & Pieridae & Pieris & napi & Braşov & Muntele Piatra Mare, $1500-2000 \mathrm{~m}$. & 1982 & May & 31 & 3 \\
\hline 7001 & Papilionoidea & Pieridae & Pieris & bryoniae & Braşov & Muntele Postăvar, 2000 m. & 1967 & July & 23 & 1 \\
\hline 7001 & Papilionoidea & Pieridae & Pieris & bryoniae & Braşov & Muntele Postăvar, 2000 m. & 1968 & May & 12 & 1 \\
\hline 7001 & Papilionoidea & Pieridae & Pieris & bryoniae & Braşov & Muntele Postăvar, 2000 m. & 1968 & June & 23 & 1 \\
\hline 7001 & Papilionoidea & Pieridae & Pieris & bryoniae & Braşov & Muntele Postăvar, 2000 m. & 1981 & May & 30 & 1 \\
\hline 7001 & Papilionoidea & Pieridae & Pieris & bryoniae & Braşov & Muntele Piatra Mare, $1500-2000 \mathrm{~m}$. & 1982 & May & 31 & 1 \\
\hline 7001 & Papilionoidea & Pieridae & Pieris & bryoniae & Braşov & Şapte Scări, muntele Piatra Mare & 1982 & July & 20 & 2 \\
\hline 7015 & Papilionoidea & Pieridae & Colias & croceus & Braşov & Dealul Lempeş, Sânpetru & 1968 & September & 4 & 1 \\
\hline 7015 & Papilionoidea & Pieridae & Colias & croceus & Braşov & Drăuşeni & 1981 & June & 20 & 1 \\
\hline 7015 & Papilionoidea & Pieridae & Colias & croceus & Hunedoara & Costeşti & 1991 & July & 8 & 2 \\
\hline 7017 & Papilionoidea & Pieridae & Colias & myrmidone & Braşov & Drăuşeni & 1968 & August & 20 & 1 \\
\hline 7017 & Papilionoidea & Pieridae & Colias & myrmidone & Braşov & Drăuşeni & 1969 & August & 17 & 1 \\
\hline 7021 & Papilionoidea & Pieridae & Colias & hyale & Braşov & Braşov, Bartolomeu & 1966 & June & 12 & 1 \\
\hline 7021 & Papilionoidea & Pieridae & Colias & hyale & Braşov & Braşov, Bartolomeu & 1969 & July & 31 & 1 \\
\hline 7021 & Papilionoidea & Pieridae & Colias & hyale & Braşov & Tâmpa, Braşov & 1982 & June & 12 & 1 \\
\hline 7021 & Papilionoidea & Pieridae & Colias & hyale & Braşov & Drăuşeni & 1966 & August & 24 & 1 \\
\hline 7021 & Papilionoidea & Pieridae & Colias & hyale & Braşov & Drăuşeni & 1969 & August & 17 & 2 \\
\hline
\end{tabular}




\begin{tabular}{|c|c|c|c|c|c|c|c|c|c|c|}
\hline $\begin{array}{c}\text { K \& R } \\
\text { LIST NR. }\end{array}$ & SUPERFAMILY & FAMILY & GENUS & SPECIES & COUNTY & LOCALITY & YEAR & MONTH & \multicolumn{2}{|c|}{ DAY NR. } \\
\hline 7021 & Papilionoidea & Pieridae & Colias & hyale & Braşov & Drăuşeni & 1979 & July & 28 & 1 \\
\hline 7021 & Papilionoidea & Pieridae & Colias & hyale & Braşov & Drăuşeni & 1982 & May & 30 & 4 \\
\hline 7022 & Papilionoidea & Pieridae & Colias & alfacariensis & Braşov & Pietrele lui Solomon, Braşov & 1969 & July & 31 & 1 \\
\hline 7024 & Papilionoidea & Pieridae & Gonepteryx & rhamni & Braşov & Dealul Lempeş, Sânpetru & 1979 & July & 14 & 1 \\
\hline 7024 & Papilionoidea & Pieridae & Gonepteryx & rhamni & Braşov & Dealul Lempeş, Sânpetru & 1979 & July & 15 & 1 \\
\hline 7024 & Papilionoidea & Pieridae & Gonepteryx & rhamni & Braşov & Dealul Bunloc, Săcele & 1991 & July & 15 & 1 \\
\hline 7024 & Papilionoidea & Pieridae & Gonepteryx & rhamni & Braşov & Drăuşeni & 1991 & July & 16 & 1 \\
\hline 7030 & Papilionoidea & Lycaenidae & Hamearis & lucina & Braşov & Homorod & 1967 & August & 27 & 1 \\
\hline 7030 & Papilionoidea & Lycaenidae & Hamearis & lucina & Braşov & Dealul Bunloc, Săcele & 1976 & May & 31 & 1 \\
\hline 7034 & Papilionoidea & Lycaenidae & Lycaena & phlaeas & Braşov & Poiana Braşov, $1000 \mathrm{~m}$. & 1968 & July & 1 & 1 \\
\hline 7034 & Papilionoidea & Lycaenidae & Lycaena & phlaeas & Braşov & Poiana Braşov, $1000 \mathrm{~m}$. & 1969 & August & 14 & 1 \\
\hline 7034 & Papilionoidea & Lycaenidae & Lycaena & phlaeas & Braşov & Drăuşeni & 1966 & August & 14 & 1 \\
\hline 7037 & Papilionoidea & Lycaenidae & Lycaena & virgaurae & Covasna & Breţcu village & 1982 & July & 4 & 2 \\
\hline 7039 & Papilionoidea & Lycaenidae & Lycaena & tityrus & Braşov & Muntele Piatra Mare, $1500-2000 \mathrm{~m}$. & 1982 & July & 20 & 2 \\
\hline 7039 & Papilionoidea & Lycaenidae & Lycaena & tityrus & Braşov & Drăuşeni & 1967 & July & 28 & 1 \\
\hline 7058 & Papilionoidea & Lycaenidae & Callophrys & rubi & Braşov & Dealul Lempeş, Sânpetru & 1981 & May & 31 & 1 \\
\hline 7058 & Papilionoidea & Lycaenidae & Callophrys & rubi & Braşov & Drăuşeni & 1998 & July & 2 & 1 \\
\hline 7062 & Papilionoidea & Lycaenidae & Satyrium & w-album & Braşov & Dealul Bunloc, Săcele & 1991 & July & 15 & 1 \\
\hline 7062 & Papilionoidea & Lycaenidae & Satyrium & w-album & Braşov & Pădurea Bogata, Hoghiz & 1998 & July & 2 & 3 \\
\hline 7062 & Papilionoidea & Lycaenidae & Satyrium & w-album & Braşov & Pădurea Bogata, Hoghiz & 1998 & July & 11 & 1 \\
\hline 7063 & Papilionoidea & Lycaenidae & Satyrium & pruni & Braşov & Poiana Braşov, 1000 m. & 1968 & May & 28 & 1 \\
\hline 7063 & Papilionoidea & Lycaenidae & Satyrium & pruni & Covasna & Vâlcele & 1981 & June & 19 & 1 \\
\hline 7064 & Papilionoidea & Lycaenidae & Satyrium & spini & Braşov & Braşov, dealul Warthe & 1968 & June & 25 & 1 \\
\hline 7064 & Papilionoidea & Lycaenidae & Satyrium & spini & Braşov & Poiana Braşov, 1000 m. & 1981 & July & 30 & 1 \\
\hline 7064 & Papilionoidea & Lycaenidae & Satyrium & spini & Braşov & Tâmpa, Braşov & 1981 & July & 25 & 1 \\
\hline 7064 & Papilionoidea & Lycaenidae & Satyrium & spini & Braşov & Tâmpa, Braşov & 1998 & July & 14 & 1 \\
\hline 7067 & Papilionoidea & Lycaenidae & Satyrium & acaciae & Braşov & Drăuşeni & 1981 & June & 20 & 2 \\
\hline 7067 & Papilionoidea & Lycaenidae & Satyrium & acaciae & Braşov & Drăuşeni & 1998 & July & 2 & 3 \\
\hline 7093 & Papilionoidea & Lycaenidae & Cupido & argiades & Braşov & Roadeş & 2002 & June & 18 & 1 \\
\hline 7093 & Papilionoidea & Lycaenidae & Cupido & argiades & Covasna & Breţcu village & 1982 & July & 24 & 1 \\
\hline 7097 & Papilionoidea & Lycaenidae & Celastrina & argiolus & Braşov & Tâmpa, Braşov & 1981 & July & 25 & 1 \\
\hline 7107 & Papilionoidea & Lycaenidae & Glaucopsyche & alexis & Braşov & Braşov, dealul Warthe & 1968 & May & 30 & 1 \\
\hline
\end{tabular}




\begin{tabular}{|c|c|c|c|c|c|c|c|c|c|c|}
\hline $\begin{array}{c}\text { K \& R } \\
\text { LIST NR. }\end{array}$ & SUPERFAMILY & FAMILY & GENUS & SPECIES & COUNTY & LOCALITY & YEAR & MONTH & \multicolumn{2}{|c|}{ DAY NR. } \\
\hline 7107 & Papilionoidea & Lycaenidae & Glaucopsyche & alexis & Braşov & Tâmpa, Braşov & 1968 & May & 30 & 1 \\
\hline 7107 & Papilionoidea & Lycaenidae & Glaucopsyche & alexis & Braşov & Tâmpa, Braşov & 1982 & June & 12 & 1 \\
\hline 7107 & Papilionoidea & Lycaenidae & Glaucopsyche & alexis & Braşov & Dealul Lempeş, Sânpetru & 1981 & May & 31 & 2 \\
\hline 7107 & Papilionoidea & Lycaenidae & Glaucopsyche & alexis & Braşov & Dealul Lempeş, Sânpetru & 1982 & May & 29 & 1 \\
\hline 7127 & Papilionoidea & Lycaenidae & Plebejus & argus & Braşov & Drăuşeni & 1981 & June & 20 & 1 \\
\hline 7127 & Papilionoidea & Lycaenidae & Plebejus & argus & Braşov & Drăuşeni & 1982 & May & 30 & 2 \\
\hline 7127 & Papilionoidea & Lycaenidae & Plebejus & argus & Braşov & Dealul Lempeş, Sânpetru & 2002 & June & 15 & 2 \\
\hline 7127 & Papilionoidea & Lycaenidae & Plebejus & argus & Braşov & Roadeş & 2002 & June & 18 & 1 \\
\hline 7128 & Papilionoidea & Lycaenidae & Plebejus & idas & Braşov & Dealul Bunloc, Săcele & 1969 & July & 18 & 1 \\
\hline 7145 & Papilionoidea & Lycaenidae & Aricia & agestis & Braşov & Braşov, Bartolomeu & 1968 & May & 26 & 1 \\
\hline 7145 & Papilionoidea & Lycaenidae & Aricia & agestis & Braşov & Teliu, 1500 m., munţii Întorsurii & 1978 & July & 2 & 1 \\
\hline 7145 & Papilionoidea & Lycaenidae & Aricia & agestis & Braşov & Dealul Lempeş, Sânpetru & 1981 & May & 31 & 1 \\
\hline 7145 & Papilionoidea & Lycaenidae & Aricia & agestis & Braşov & Dealul Lempeş, Sânpetru & 2002 & June & 17 & 1 \\
\hline 7146 & Papilionoidea & Lycaenidae & Aricia & artaxerxes & Braşov & Dealul Lempeş, Sânpetru & 1981 & May & 31 & 1 \\
\hline 7152 & Papilionoidea & Lycaenidae & Cyaniris & semiargus & Braşov & Braşov, dealul Warthe & 1968 & May & 26 & 1 \\
\hline 7152 & Papilionoidea & Lycaenidae & Cyaniris & semiargus & Braşov & Tâmpa, Braşov & 1981 & May & 30 & 1 \\
\hline 7152 & Papilionoidea & Lycaenidae & Cyaniris & semiargus & Braşov & Tâmpa, Braşov & 1982 & June & 12 & 1 \\
\hline 7152 & Papilionoidea & Lycaenidae & Cyaniris & semiargus & Braşov & Dealul Bunloc, Săcele & 1980 & June & 21 & 1 \\
\hline 7152 & Papilionoidea & Lycaenidae & Cyaniris & semiargus & Braşov & Dealul Lempeş, Sânpetru & 1982 & May & 29 & 1 \\
\hline 7152 & Papilionoidea & Lycaenidae & Cyaniris & semiargus & Braşov & Teliu, 1500 m., munţii Întorsurii & 1978 & July & 2 & 3 \\
\hline 7152 & Papilionoidea & Lycaenidae & Cyaniris & semiargus & Braşov & Drăuşeni & 1982 & May & 30 & 1 \\
\hline 7152 & Papilionoidea & Lycaenidae & Cyaniris & semiargus & Covasna & Vâlcele & 1981 & June & 19 & 1 \\
\hline 7172 & Papilionoidea & Lycaenidae & Lysandra & bellargus & Braşov & Braşov, Bartolomeu & 1968 & May & 30 & 1 \\
\hline 7172 & Papilionoidea & Lycaenidae & Lysandra & bellargus & Braşov & Dealul Lempeş, Sânpetru & 1969 & August & 25 & 1 \\
\hline 7172 & Papilionoidea & Lycaenidae & Lysandra & bellargus & Braşov & Roadeş & 2002 & June & 18 & 2 \\
\hline 7173 & Papilionoidea & Lycaenidae & Lysandra & coridon & Braşov & Braşov, dealul Warthe & 1966 & August & 12 & 1 \\
\hline 7173 & Papilionoidea & Lycaenidae & Lysandra & coridon & Braşov & Braşov, dealul Warthe & 1968 & July & 8 & 1 \\
\hline 7173 & Papilionoidea & Lycaenidae & Lysandra & coridon & Braşov & Tâmpa, Braşov & 1967 & September & 10 & 3 \\
\hline 7173 & Papilionoidea & Lycaenidae & Lysandra & coridon & Braşov & Tâmpa, Braşov & 1978 & October & 16 & 2 \\
\hline 7173 & Papilionoidea & Lycaenidae & Lysandra & coridon & Braşov & Tâmpa, Braşov & 1981 & September & 24 & 1 \\
\hline 7173 & Papilionoidea & Lycaenidae & Lysandra & coridon & Braşov & Tâmpa, Braşov & 1982 & September & 5 & 3 \\
\hline 7157 & Papilionoidea & Lycaenidae & Polyommatus & dorylas & Braşov & Teliu, 1500 m., munţii Întorsurii & 1978 & July & 2 & 2 \\
\hline
\end{tabular}




\begin{tabular}{|c|c|c|c|c|c|c|c|c|c|c|}
\hline $\begin{array}{c}\text { K \& R } \\
\text { LIST NR. }\end{array}$ & SUPERFAMILY & FAMILY & GENUS & SPECIES & COUNTY & LOCALITY & YEAR & MONTH & \multicolumn{2}{|c|}{ DAY NR. } \\
\hline 7162 & Papilionoidea & Lycaenidae & Polyommatus & thersites & Braşov & Braşov, Bartolomeu & 1967 & May & 30 & 1 \\
\hline 7162 & Papilionoidea & Lycaenidae & Polyommatus & thersites & Braşov & Dealul Lempeş, Sânpetru & 1982 & May & 29 & 1 \\
\hline 7163 & Papilionoidea & Lycaenidae & Polyommatus & icarus & Braşov & Dealul Lempeş, Sânpetru & 1981 & May & 31 & 1 \\
\hline 7163 & Papilionoidea & Lycaenidae & Polyommatus & icarus & Braşov & Homorod & 1967 & July & 1 & 2 \\
\hline 7163 & Papilionoidea & Lycaenidae & Polyommatus & icarus & Braşov & Roadeş & 2002 & June & 18 & 1 \\
\hline 7307 & Papilionoidea & Nymphalidae & Pararge & aegeria & Braşov & Braşov, Dealul Warthe & 1969 & August & 7 & 1 \\
\hline 7307 & Papilionoidea & Nymphalidae & Pararge & aegeria & Braşov & Pietrele lui Solomon, Braşov & 1968 & June & 3 & 2 \\
\hline 7309 & Papilionoidea & Nymphalidae & Lasiommata & megera & Braşov & Homorod & 1967 & June & 25 & 1 \\
\hline 7309 & Papilionoidea & Nymphalidae & Lasiommata & megera & Braşov & Dealul Lempeş, Sânpetru & 1979 & July & 14 & 1 \\
\hline 7309 & Papilionoidea & Nymphalidae & Lasiommata & megera & Braşov & Tâmpa, Braşov & 1981 & July & 25 & 2 \\
\hline 7309 & Papilionoidea & Nymphalidae & Lasiommata & megera & Hunedoara & Costeşti & 1991 & July & 8 & 1 \\
\hline 7312 & Papilionoidea & Nymphalidae & Lasiommata & maera & Braşov & Pietrele lui Solomon, Braşov & 1968 & May & 26 & 1 \\
\hline 7312 & Papilionoidea & Nymphalidae & Lasiommata & maera & Braşov & Pietrele lui Solomon, Braşov & 1968 & June & 3 & 1 \\
\hline 7312 & Papilionoidea & Nymphalidae & Lasiommata & maera & Braşov & Poiana Braşov, 1000 m. & 1968 & June & 5 & 1 \\
\hline 7312 & Papilionoidea & Nymphalidae & Lasiommata & maera & Braşov & Muntele Postăvar, 2000 m. & 1968 & May & 30 & 1 \\
\hline 7312 & Papilionoidea & Nymphalidae & Lasiommata & maera & Braşov & Dealul Bunloc, Săcele & 1991 & July & 15 & 1 \\
\hline 7312 & Papilionoidea & Nymphalidae & Lasiommata & maera & Hunedoara & Costeşti & 1991 & July & 8 & 1 \\
\hline 7315 & Papilionoidea & Nymphalidae & Lopinga & achine & Covasna & Bodoc Mt. & 1990 & June & 30 & 1 \\
\hline 7325 & Papilionoidea & Nymphalidae & Coenonympha & arcania & Covasna & Vâlcele & 1981 & June & 19 & 1 \\
\hline 7326 & Papilionoidea & Nymphalidae & Coenonympha & glycerion & Braşov & Poiana Braşov, 1000 m. & 1968 & June & 17 & 1 \\
\hline 7326 & Papilionoidea & Nymphalidae & Coenonympha & glycerion & Braşov & Dealul Lempeş, Sânpetru & 2002 & June & 15 & 2 \\
\hline 7326 & Papilionoidea & Nymphalidae & Coenonympha & glycerion & Covasna & Vâlcele & 1981 & June & 19 & 3 \\
\hline 7326 & Papilionoidea & Nymphalidae & Coenonympha & glycerion & Hunedoara & Sibişel & 2001 & July & 10 & 2 \\
\hline 7326 & Papilionoidea & Nymphalidae & Coenonympha & glycerion & Hunedoara & Sibişel & 2003 & July & 1 & 1 \\
\hline 7334 & Papilionoidea & Nymphalidae & Coenonympha & pamphilus & Braşov & Dealul Lempeş, Sânpetru & 1981 & May & 31 & 1 \\
\hline 7334 & Papilionoidea & Nymphalidae & Coenonympha & pamphilus & Braşov & Drăuşeni & 1981 & June & 20 & 1 \\
\hline 7334 & Papilionoidea & Nymphalidae & Coenonympha & pamphilus & Covasna & Vâlcele & 1981 & June & 19 & 1 \\
\hline 7334 & Papilionoidea & Nymphalidae & Coenonympha & pamphilus & Hunedoara & Sibişel & 2002 & July & 10 & 2 \\
\hline 7334 & Papilionoidea & Nymphalidae & Coenonympha & pamphilus & Hunedoara & Sibişel & 2003 & July & 1 & 1 \\
\hline 7360 & Papilionoidea & Nymphalidae & Erebia & ligea & Braşov & Pietrele lui Solomon, Braşov & 1979 & July & 8 & 1 \\
\hline 7360 & Papilionoidea & Nymphalidae & Erebia & ligea & Braşov & Muntele Postăvar, 2000 m. & 1979 & July & 8 & 1 \\
\hline 7360 & Papilionoidea & Nymphalidae & Erebia & ligea & Braşov & Tâmpa, Braşov & 1981 & July & 25 & 1 \\
\hline
\end{tabular}




\begin{tabular}{|c|c|c|c|c|c|c|c|c|c|c|}
\hline $\begin{array}{c}\text { K \& R } \\
\text { LIST NR. }\end{array}$ & SUPERFAMILY & FAMILY & GENUS & SPECIES & COUNTY & LOCALITY & YEAR & MONTH & \multicolumn{2}{|c|}{ DAY NR. } \\
\hline 7360 & Papilionoidea & Nymphalidae & Erebia & ligea & Braşov & Dealul Bunloc, Săcele & 1981 & June & 29 & 2 \\
\hline 7360 & Papilionoidea & Nymphalidae & Erebia & ligea & Braşov & Dealul Bunloc, Săcele & 1991 & July & 15 & 1 \\
\hline 7360 & Papilionoidea & Nymphalidae & Erebia & ligea & Braşov & Şapte Scări, muntele Piatra Mare & 1981 & August & 1 & 1 \\
\hline 7360 & Papilionoidea & Nymphalidae & Erebia & ligea & Covasna & Breţcu village & 1982 & July & 24 & 1 \\
\hline 7360 & Papilionoidea & Nymphalidae & Erebia & ligea & Hunedoara & Sibişel & 2003 & July & 1 & 1 \\
\hline 7361 & Papilionoidea & Nymphalidae & Erebia & euryale & Braşov & Braşov, dealul Warthe & 1969 & August & 7 & 1 \\
\hline 7361 & Papilionoidea & Nymphalidae & Erebia & euryale & Braşov & Muntele Postăvar, 2000 m. & 1969 & August & 7 & 1 \\
\hline 7361 & Papilionoidea & Nymphalidae & Erebia & euryale & Braşov & Pietrele lui Solomon, Braşov & 1979 & July & 8 & 1 \\
\hline 7361 & Papilionoidea & Nymphalidae & Erebia & euryale & Braşov & Poiana Braşov, 1000 m. & 1979 & July & 8 & 4 \\
\hline 7361 & Papilionoidea & Nymphalidae & Erebia & euryale & Braşov & Dealul Bunloc, Săcele & 1979 & July & 7 & 1 \\
\hline 7361 & Papilionoidea & Nymphalidae & Erebia & euryale & Braşov & Dealul Bunloc, Săcele & 1981 & August & 1 & 2 \\
\hline 7361 & Papilionoidea & Nymphalidae & Erebia & euryale & Braşov & Dealul Bunloc, Săcele & 1991 & July & 15 & 1 \\
\hline 7361 & Papilionoidea & Nymphalidae & Erebia & euryale & Braşov & Şapte Scări, muntele Piatra Mare & 1981 & August & 1 & 4 \\
\hline 7372 & Papilionoidea & Nymphalidae & Erebia & aethiops & Hunedoara & Sibişel & 2003 & July & 16 & 3 \\
\hline 7379 & Papilionoidea & Nymphalidae & Erebia & medusa & Braşov & Pietrele lui Solomon, Braşov & 1968 & May & 20 & 1 \\
\hline 7379 & Papilionoidea & Nymphalidae & Erebia & medusa & Braşov & Tâmpa, Braşov & 1981 & May & 19 & 1 \\
\hline 7379 & Papilionoidea & Nymphalidae & Erebia & medusa & Braşov & Tâmpa, Braşov & 1981 & May & 29 & 2 \\
\hline 7379 & Papilionoidea & Nymphalidae & Erebia & medusa & Braşov & Şapte Scări, muntele Piatra Mare & 1982 & July & 20 & 1 \\
\hline 7379 & Papilionoidea & Nymphalidae & Erebia & medusa & Braşov & Pădurea Bogata, Hoghiz & 1979 & May & 27 & 3 \\
\hline 7379 & Papilionoidea & Nymphalidae & Erebia & medusa & Braşov & Fundata, $1290 \mathrm{~m}$. & 2007 & May & 24 & 2 \\
\hline 7344 & Papilionoidea & Nymphalidae & Aphantopus & hyperantus & Braşov & Tâmpa, Braşov & 1981 & July & 25 & 1 \\
\hline 7344 & Papilionoidea & Nymphalidae & Aphantopus & hyperantus & Braşov & Dealul Lempeş, Sânpetru & 1981 & July & 23 & 3 \\
\hline 7350 & Papilionoidea & Nymphalidae & Maniola & jurtina & Braşov & Drăuşeni & 1969 & July & 17 & 1 \\
\hline 7350 & Papilionoidea & Nymphalidae & Maniola & jurtina & Hunedoara & Costeşti & 1991 & July & 8 & 1 \\
\hline 7415 & Papilionoidea & Nymphalidae & Melanargia & galathea & Braşov & Braşov, Bartolomeu & 1968 & June & 25 & 1 \\
\hline 7427 & Papilionoidea & Nymphalidae & Minois & dryas & Braşov & Dealul Lempeş, Sânpetru & 1979 & July & 14 & 1 \\
\hline 7427 & Papilionoidea & Nymphalidae & Minois & dryas & Braşov & Dealul Lempeş, Sânpetru & 1979 & July & 29 & 4 \\
\hline 7449 & Papilionoidea & Nymphalidae & Chazara & briseis & Braşov & Drăuşeni & 1966 & July & 24 & 1 \\
\hline 7449 & Papilionoidea & Nymphalidae & Chazara & briseis & Braşov & Drăuşeni & 1969 & August & 17 & 3 \\
\hline 7436 & Papilionoidea & Nymphalidae & Hipparchia & semele & Braşov & Drăuşeni & 1968 & August & 13 & 1 \\
\hline 7202 & Papilionoidea & Nymphalidae & Argynnis & paphia & Braşov & Pietrele lui Solomon, Braşov & 1969 & July & 17 & 1 \\
\hline 7202 & Papilionoidea & Nymphalidae & Argynnis & paphia & Braşov & Pietrele lui Solomon, Braşov & 1973 & August & 28 & 1 \\
\hline
\end{tabular}




\begin{tabular}{|c|c|c|c|c|c|c|c|c|c|c|}
\hline $\begin{array}{c}\text { K \& R } \\
\text { LIST NR. }\end{array}$ & SUPERFAMILY & FAMILY & GENUS & SPECIES & COUNTY & LOCALITY & YEAR & MONTH & \multicolumn{2}{|c|}{ DAY NR. } \\
\hline 7202 & Papilionoidea & Nymphalidae & Argynnis & paphia & Braşov & Dealul Bunloc, Săcele & 1968 & July & 1 & 1 \\
\hline 7202 & Papilionoidea & Nymphalidae & Argynnis & paphia & Braşov & Dealul Bunloc, Săcele & 1969 & July & 18 & 1 \\
\hline 7202 & Papilionoidea & Nymphalidae & Argynnis & paphia & Braşov & Şapte Scări, muntele Piatra Mare & 1981 & August & 1 & 1 \\
\hline 7208 & Papilionoidea & Nymphalidae & Argynnis & laodice & Sibiu & Richiria & 1989 & July & 20 & 1 \\
\hline 7204 & Papilionoidea & Nymphalidae & Speyeria & aglaja & Braşov & Poiana Braşov, $1000 \mathrm{~m}$. & 1968 & June & 17 & 1 \\
\hline 7204 & Papilionoidea & Nymphalidae & Speyeria & aglaja & Braşov & Dealul Bunloc, Săcele & 1969 & July & 18 & 2 \\
\hline 7204 & Papilionoidea & Nymphalidae & Speyeria & aglaja & Braşov & Fundata, $1290 \mathrm{~m}$. & 1982 & July & 19 & 2 \\
\hline 7204 & Papilionoidea & Nymphalidae & Speyeria & aglaja & Braşov & Fundata, $1290 \mathrm{~m}$. & 1998 & July & 13 & 1 \\
\hline 7204 & Papilionoidea & Nymphalidae & Speyeria & aglaja & Covasna & Vâlcele & 1981 & June & 19 & 1 \\
\hline 7204 & Papilionoidea & Nymphalidae & Speyeria & aglaja & Hunedoara & Costeşti & 1991 & July & 8 & 1 \\
\hline 7205 & Papilionoidea & Nymphalidae & Fabriciana & adippe & Braşov & Poiana Braşov, $1000 \mathrm{~m}$. & 1967 & July & 23 & 1 \\
\hline 7205 & Papilionoidea & Nymphalidae & Fabriciana & adippe & Braşov & Pădurea Bogata, Hoghiz & 1998 & July & 11 & 1 \\
\hline 7206 & Papilionoidea & Nymphalidae & Fabriciana & niobe & Braşov & Zizin & 1967 & August & 7 & 1 \\
\hline 7206 & Papilionoidea & Nymphalidae & Fabriciana & niobe & Braşov & Poiana Braşov, 1000 m. & 1968 & June & 23 & 1 \\
\hline 7206 & Papilionoidea & Nymphalidae & Fabriciana & niobe & Braşov & Dealul Bunloc, Săcele & 1969 & July & 18 & 2 \\
\hline 7206 & Papilionoidea & Nymphalidae & Fabriciana & niobe & Braşov & Dealul Bunloc, Săcele & 1969 & July & 24 & 2 \\
\hline 7206 & Papilionoidea & Nymphalidae & Fabriciana & niobe & Braşov & Vlădeni & 1978 & July & 1 & 1 \\
\hline 7206 & Papilionoidea & Nymphalidae & Fabriciana & niobe & Braşov & Tâmpa, Braşov & 1981 & July & 25 & 1 \\
\hline 7206 & Papilionoidea & Nymphalidae & Fabriciana & niobe & Covasna & Breţcu village & 1982 & July & 23 & 3 \\
\hline 7210 & Papilionoidea & Nymphalidae & Issoria & lathonia & Braşov & Braşov, Bartolomeu & 1969 & July & 12 & 1 \\
\hline 7213 & Papilionoidea & Nymphalidae & Brenthis & ino & Braşov & Vlădeni & 1978 & July & 1 & 1 \\
\hline 7220 & Papilionoidea & Nymphalidae & Boloria & euphrosyne & Braşov & Muntele Postăvar, 2000 m. & 1967 & April & 29 & 1 \\
\hline 7220 & Papilionoidea & Nymphalidae & Boloria & euphrosyne & Braşov & Pietrele lui Solomon, Braşov & 1968 & May & 26 & 1 \\
\hline 7220 & Papilionoidea & Nymphalidae & Boloria & euphrosyne & Braşov & Poiana Braşov, 1000 m. & 1968 & June & 19 & 1 \\
\hline 7220 & Papilionoidea & Nymphalidae & Boloria & euphrosyne & Braşov & Vlădeni & 1978 & July & 1 & 1 \\
\hline 7220 & Papilionoidea & Nymphalidae & Boloria & euphrosyne & Braşov & Dealul Lempeş, Sânpetru & 1982 & May & 29 & 1 \\
\hline 7220 & Papilionoidea & Nymphalidae & Boloria & euphrosyne & Braşov & Muntele Piatra Mare, $1500-2000 \mathrm{~m}$. & 1982 & May & 31 & 2 \\
\hline 7220 & Papilionoidea & Nymphalidae & Boloria & euphrosyne & Covasna & Vâlcele & 1981 & June & 19 & 1 \\
\hline 7222 & Papilionoidea & Nymphalidae & Boloria & selene & Braşov & Poiana Braşov, $1000 \mathrm{~m}$. & 1969 & August & 7 & 1 \\
\hline 7222 & Papilionoidea & Nymphalidae & Boloria & selene & Covasna & Vâlcele & 1981 & June & 19 & 3 \\
\hline 7228 & Papilionoidea & Nymphalidae & Boloria & dia & Braşov & Teliu, 1500 m., munţii Întorsurii & 1978 & July & 30 & 1 \\
\hline 7287 & Papilionoidea & Nymphalidae & Limenitis & camilla & Braşov & Pietrele lui Solomon, Braşov & 1969 & July & 17 & 1 \\
\hline
\end{tabular}




\begin{tabular}{|c|c|c|c|c|c|c|c|c|c|c|}
\hline $\begin{array}{c}\text { K \& R } \\
\text { LIST NR. }\end{array}$ & SUPERFAMILY & FAMILY & GENUS & SPECIES & COUNTY & LOCALITY & YEAR & MONTH & \multicolumn{2}{|c|}{ DAY NR } \\
\hline 7290 & Papilionoidea & Nymphalidae & Neptis & sappho & Sibiu & Richiria & 1993 & July & 12 & 2 \\
\hline 7291 & Papilionoidea & Nymphalidae & Neptis & rivularis & Braşov & Braşov, Bartolomeu & 1968 & May & 30 & 1 \\
\hline 7291 & Papilionoidea & Nymphalidae & Neptis & rivularis & Braşov & Tâmpa, Braşov & 1981 & July & 25 & 4 \\
\hline 7291 & Papilionoidea & Nymphalidae & Neptis & rivularis & Braşov & Tâmpa, Braşov & 1991 & July & 17 & 1 \\
\hline 7291 & Papilionoidea & Nymphalidae & Neptis & rivularis & Braşov & Braşov, dealul Warthe & 1991 & July & 17 & 1 \\
\hline 7298 & Papilionoidea & Nymphalidae & Apatura & ilia & Braşov & Pădurea Bogata, Hoghiz & 1991 & July & 16 & 2 \\
\hline 7299 & Papilionoidea & Nymphalidae & Apatura & iris & Braşov & Tâmpa, Braşov & 1967 & July & 14 & 1 \\
\hline 7299 & Papilionoidea & Nymphalidae & Apatura & iris & Braşov & Tâmpa, Braşov & 1968 & July & 1 & 1 \\
\hline 7299 & Papilionoidea & Nymphalidae & Apatura & iris & Braşov & Tâmpa, Braşov & 1981 & July & 25 & 1 \\
\hline 7299 & Papilionoidea & Nymphalidae & Apatura & iris & Braşov & Şapte Scări, muntele Piatra Mare & 1982 & August & 14 & 1 \\
\hline 7299 & Papilionoidea & Nymphalidae & Apatura & iris & Braşov & Dealul Bunloc, Săcele & 1991 & July & 15 & 2 \\
\hline 7299 & Papilionoidea & Nymphalidae & Apatura & iris & Covasna & Breţcu village & 1982 & July & 24 & 3 \\
\hline 7299 & Papilionoidea & Nymphalidae & Apatura & iris & Hunedoara & Sibişel & 1999 & July & 20 & 1 \\
\hline 7257 & Papilionoidea & Nymphalidae & Nymphalis & antiopa & Braşov & Pietrele lui Solomon, Braşov & 1969 & April & 27 & 1 \\
\hline 7257 & Papilionoidea & Nymphalidae & Nymphalis & antiopa & Hunedoara & Sibişel & 1999 & August & 2 & 1 \\
\hline 7258 & Papilionoidea & Nymphalidae & Nymphalis & polychloros & Braşov & Tâmpa, Braşov & 1969 & July & 24 & 1 \\
\hline 7250 & Papilionoidea & Nymphalidae & Aglais & urticae & Braşov & Braşov, Bartolomeu & 1978 & Ocober & 28 & 1 \\
\hline 7250 & Papilionoidea & Nymphalidae & Aglais & urticae & Braşov & Dealul Lempeş, Sânpetru & 1979 & July & 14 & 1 \\
\hline 7250 & Papilionoidea & Nymphalidae & Aglais & urticae & Braşov & Dealul Lempeş, Sânpetru & 1979 & July & 29 & 1 \\
\hline 7250 & Papilionoidea & Nymphalidae & Aglais & urticae & Braşov & Dealul Bunloc, Săcele & 1991 & July & 15 & 1 \\
\hline 7248 & Papilionoidea & Nymphalidae & Aglais & io & Braşov & Drăuşeni & 1969 & August & 14 & 1 \\
\hline 7248 & Papilionoidea & Nymphalidae & Aglais & io & Braşov & Dealul Bunloc, Săcele & 1991 & July & 15 & 1 \\
\hline 7243 & Papilionoidea & Nymphalidae & Vanessa & atalanta & Braşov & Drăuşeni & 1966 & August & 14 & 1 \\
\hline 7243 & Papilionoidea & Nymphalidae & Vanessa & atalanta & Braşov & Braşov, dealul Warthe & 1970 & July & 9 & 1 \\
\hline 7245 & Papilionoidea & Nymphalidae & Vanessa & cardui & Braşov & Braşov, Bartolomeu & 1968 & July & 12 & 1 \\
\hline 7252 & Papilionoidea & Nymphalidae & Polygonia & c-album & Braşov & Dealul Lempeş, Sânpetru & 1979 & July & 14 & 2 \\
\hline 7255 & Papilionoidea & Nymphalidae & Araschnia & levana & Braşov & Voila & 1968 & May & 15 & 1 \\
\hline 7270 & Papilionoidea & Nymphalidae & Melitaea & cinxia & Braşov & Dealul Lempeş, Sânpetru & 1981 & May & 31 & 3 \\
\hline 7275 & Papilionoidea & Nymphalidae & Melitaea & didyma & Braşov & Tâmpa, Braşov & 1976 & August & 24 & 1 \\
\hline 7275 & Papilionoidea & Nymphalidae & Melitaea & didyma & Braşov & Tâmpa, Braşov & 1981 & July & 25 & 1 \\
\hline 7275 & Papilionoidea & Nymphalidae & Melitaea & didyma & Braşov & Dealul Lempeş, Sânpetru & 1979 & July & 29 & 1 \\
\hline 7275 & Papilionoidea & Nymphalidae & Melitaea & didyma & Sibiu & Richiria & 1989 & July & 20 & 1 \\
\hline
\end{tabular}




\begin{tabular}{|c|c|c|c|c|c|c|c|c|c|c|}
\hline $\begin{array}{c}\text { K \& R } \\
\text { LIST NR. }\end{array}$ & SUPERFAMILY & FAMILY & GENUS & SPECIES & COUNTY & LOCALITY & YEAR & MONTH & DAY & NR. \\
\hline 7283 & Papilionoidea & Nymphalidae & Melitaea & athalia & Braşov & Dealul Lempeş, Sânpetru & 1981 & May & 31 & 2 \\
\hline 7280 & Papilionoidea & Nymphalidae & Melitaea & aurelia & Braşov & Teliu, 1500 m., munții Întorsurii & 1978 & July & 2 & 1 \\
\hline 7280 & Papilionoidea & Nymphalidae & Melitaea & aurelia & Covasna & Vâlcele & 1981 & June & 19 & 1 \\
\hline 5611 & Pyraloidea & Pyralidae & Synaphe & moldavica & Braşov & Muntele Postăvar, 2000 m. & 1966 & August & 3 & 1 \\
\hline 5620 & Pyraloidea & Pyralidae & Synaphe & punctalis & Hunedoara & Sibişel & 1999 & August & 11 & 1 \\
\hline 5620 & Pyraloidea & Pyralidae & Synaphe & punctalis & Hunedoara & Sibişel & 2002 & July & 22 & 1 \\
\hline 5625 & Pyraloidea & Pyralidae & Pyralis & regalis & Hunedoara & Sibişel & 2003 & June & 27 & 1 \\
\hline 5625 & Pyraloidea & Pyralidae & Pyralis & regalis & Hunedoara & Sibişel & 2003 & July & 1 & 3 \\
\hline 5633 & Pyraloidea & Pyralidae & Aglossa & pinguinalis & Braşov & Homorod & 1981 & June & 20 & 1 \\
\hline 5652 & Pyraloidea & Pyralidae & Hypsopygia & costalis & Hunedoara & Sibişel & 2003 & July & 3 & 1 \\
\hline 5656 & Pyraloidea & Pyralidae & Hypsopygia & rubidalis & Hunedoara & Sibişel & 2002 & July & 15 & 1 \\
\hline 5658 & Pyraloidea & Pyralidae & Hypsopygia & glaucinalis & Hunedoara & Sibişel & 2001 & August & 27 & 1 \\
\hline 5751 & Pyraloidea & Pyralidae & Oncocera & semirubella & Braşov & Tâmpa, Braşov & 1967 & August & 2 & 1 \\
\hline 5732 & Pyraloidea & Pyralidae & Selagia & argyrella & Braşov & Dealul Lempeş, Sânpetru & 2003 & August & 5 & 2 \\
\hline 5796 & Pyraloidea & Pyralidae & Phycita & roborella & Hunedoara & Sibişel & 2000 & August & 4 & 1 \\
\hline 5781 & Pyraloidea & Pyralidae & Dioryctria & sylvestrella & Hunedoara & Sibişel & 2000 & August & 7 & 1 \\
\hline 5784 & Pyraloidea & Pyralidae & Dioryctria & abietella & Hunedoara & Sibişel & 2000 & August & 14 & 1 \\
\hline 5784 & Pyraloidea & Pyralidae & Dioryctria & abietella & Hunedoara & Sibişel & 2003 & July & 1 & 1 \\
\hline 5742 & Pyraloidea & Pyralidae & Etiella & zinckenella & Hunedoara & Sibişel & 2001 & July & 10 & 1 \\
\hline 5811 & Pyraloidea & Pyralidae & Hypochalcia & ahenella & Braşov & Dealul Lempeş, Sânpetru & 2002 & June & 17 & 1 \\
\hline 5854 & Pyraloidea & Pyralidae & Acrobasis & repandana & Hunedoara & Sibişel & 2003 & June & 29 & 1 \\
\hline 5869 & Pyraloidea & Pyralidae & Acrobasis & consociella & Hunedoara & Sibişel & 2000 & August & 7 & 1 \\
\hline 5856 & Pyraloidea & Pyralidae & Acrobasis & advenella & Hunedoara & Sibişel & 2000 & August & 9 & 1 \\
\hline 5880 & Pyraloidea & Pyralidae & Episcythrastis & tetricella & Braşov & Dealul Lempeş, Sânpetru & 2002 & June & 15 & 1 \\
\hline 5898 & Pyraloidea & Pyralidae & Myelois & circumvoluta & Braşov & Poiana Braşov, 1000 m. & 1972 & July & 4 & 1 \\
\hline 6072 & Pyraloidea & Pyralidae & Homoeosoma & sinuella & Braşov & Dealul Lempeş, Sânpetru & 2002 & June & 15 & 10 \\
\hline 6087 & Pyraloidea & Pyralidae & Phycitodes & binaevella & Hunedoara & Sibişel & 2000 & August & 11 & 1 \\
\hline 6105 & Pyraloidea & Pyralidae & Ephestia & kuehniella & Hunedoara & Sibişel & 2002 & July & 19 & 1 \\
\hline 6119 & Pyraloidea & Pyralidae & Cadra & cautella & Braşov & Braşov, Bartolomeu & 2007 & May & 27 & 1 \\
\hline 6166 & Pyraloidea & Crambidae & Scoparia & basistrigalis & Hunedoara & Sibişel & 1999 & August & 12 & 1 \\
\hline 6166 & Pyraloidea & Crambidae & Scoparia & basistrigalis & Hunedoara & Sibişel & 2001 & August & 10 & 2 \\
\hline 6166 & Pyraloidea & Crambidae & Scoparia & basistrigalis & Hunedoara & Sibişel & 2003 & June & 19 & 1 \\
\hline
\end{tabular}




\begin{tabular}{|c|c|c|c|c|c|c|c|c|c|c|}
\hline $\begin{array}{c}\text { K \& R } \\
\text { LIST NR. }\end{array}$ & SUPERFAMILY & FAMILY & GENUS & SPECIES & COUNTY & LOCALITY & YEAR & MONTH & \multicolumn{2}{|c|}{ DAY NR. } \\
\hline 6166 & Pyraloidea & Crambidae & Scoparia & basistrigalis & Hunedoara & Sibişel & 2003 & July & 2 & 1 \\
\hline 6172 & Pyraloidea & Crambidae & Scoparia & pyralella & Hunedoara & Sibişel & 2001 & August & 10 & 1 \\
\hline 6180 & Pyraloidea & Crambidae & Eudonia & lacustrata & Braşov & Pădurea Bogata, Hoghiz & 2002 & June & 10 & 1 \\
\hline 6180 & Pyraloidea & Crambidae & Eudonia & lacustrata & Hunedoara & Sibişel & 2003 & June & 22 & 3 \\
\hline 6180 & Pyraloidea & Crambidae & Eudonia & lacustrata & Hunedoara & Sibişel & 2003 & June & 29 & 1 \\
\hline 6180 & Pyraloidea & Crambidae & Eudonia & lacustrata & Hunedoara & Sibişel & 2003 & July & 1 & 1 \\
\hline 6193 & Pyraloidea & Crambidae & Eudonia & truncicolella & Hunedoara & Sibişel & 2002 & July & 15 & 1 \\
\hline 6207 & Pyraloidea & Crambidae & Euchromius & ocellea & Hunedoara & Sibişel & 2001 & July & 10 & 1 \\
\hline 6241 & Pyraloidea & Crambidae & Chrysoteuchia & culmella & Braşov & Dealul Lempeş, Sânpetru & 2002 & June & 15 & 1 \\
\hline 6241 & Pyraloidea & Crambidae & Chrysoteuchia & culmella & Covasna & Vâlcele & 1981 & June & 19 & 1 \\
\hline 6243 & Pyraloidea & Crambidae & Crambus & pascuella & Braşov & Tâmpa, Braşov & 1982 & June & 12 & 2 \\
\hline 6243 & Pyraloidea & Crambidae & Crambus & pascuella & Braşov & Braşov, dealul Warthe & 1982 & June & 14 & 1 \\
\hline 6243 & Pyraloidea & Crambidae & Crambus & pascuella & Braşov & Dealul Lempeş, Sânpetru & 2002 & June & 15 & 8 \\
\hline 6243 & Pyraloidea & Crambidae & Crambus & pascuella & Braşov & Pădurea Bogata, Hoghiz & 2002 & June & 10 & 1 \\
\hline 6243 & Pyraloidea & Crambidae & Crambus & pascuella & Braşov & Drăuşeni & 1981 & June & 20 & 1 \\
\hline 6243 & Pyraloidea & Crambidae & Crambus & pascuella & Braşov & Drăuşeni & 1982 & May & 30 & 1 \\
\hline 6250 & Pyraloidea & Crambidae & Crambus & pratella & Braşov & Dealul Lempeş, Sânpetru & 2002 & June & 17 & 2 \\
\hline 6251 & Pyraloidea & Crambidae & Crambus & lathoniellus & Braşov & Braşov, dealul Warthe & 1981 & May & 31 & 1 \\
\hline 6251 & Pyraloidea & Crambidae & Crambus & lathoniellus & Braşov & Braşov, dealul Warthe & 1982 & June & 14 & 1 \\
\hline 6251 & Pyraloidea & Crambidae & Crambus & lathoniellus & Braşov & Dealul Lempeş, Sânpetru & 1982 & May & 29 & 1 \\
\hline 6251 & Pyraloidea & Crambidae & Crambus & lathoniellus & Braşov & Dealul Lempeş, Sânpetru & 2002 & June & 15 & 1 \\
\hline 6251 & Pyraloidea & Crambidae & Crambus & lathoniellus & Braşov & Drăuşeni & 1982 & May & 30 & 1 \\
\hline 6251 & Pyraloidea & Crambidae & Crambus & lathoniellus & Braşov & Fundata, $1290 \mathrm{~m}$. & 1998 & July & 13 & 1 \\
\hline 6253 & Pyraloidea & Crambidae & Crambus & perlella & Braşov & Drăuşeni & 1981 & June & 20 & 1 \\
\hline 6253 & Pyraloidea & Crambidae & Crambus & perlella & Braşov & Drăuşeni & 1981 & July & 30 & 1 \\
\hline 6253 & Pyraloidea & Crambidae & Crambus & perlella & Hunedoara & Sibişel & 2001 & September & 5 & 1 \\
\hline 6258 & Pyraloidea & Crambidae & Agriphila & tristella & Covasna & Vâlcele & 2003 & August & 5 & 1 \\
\hline 6258 & Pyraloidea & Crambidae & Agriphila & tristella & Braşov & Tâmpa, Braşov & 2009 & September & 10 & 1 \\
\hline 6258 & Pyraloidea & Crambidae & Agriphila & tristella & Braşov & Pădurea Bogata, Hoghiz & 1982 & August & 29 & 1 \\
\hline 6260 & Pyraloidea & Crambidae & Agriphila & inquinatella & Braşov & Tâmpa, Braşov & 2009 & September & 10 & 2 \\
\hline 6266 & Pyraloidea & Crambidae & Agriphila & selasella & Hunedoara & Sibişel & 1999 & June & 10 & 1 \\
\hline 6275 & Pyraloidea & Crambidae & Agriphila & geniculea & Hunedoara & Sibişel & 2000 & August & 13 & 1 \\
\hline
\end{tabular}




\begin{tabular}{|c|c|c|c|c|c|c|c|c|c|c|}
\hline $\begin{array}{c}\text { K \& R } \\
\text { LIST NR. }\end{array}$ & SUPERFAMILY & FAMILY & GENUS & SPECIES & COUNTY & LOCALITY & YEAR & MONTH & \multicolumn{2}{|c|}{ DAY NR. } \\
\hline 6280 & Pyraloidea & Crambidae & Catoptria & permutatellus & Braşov & Poiana Braşov, 1000 m. & 1981 & July & 25 & 1 \\
\hline 6282 & Pyraloidea & Crambidae & Catoptria & myella & Hunedoara & Sibişel & 1999 & August & 16 & 1 \\
\hline 6282 & Pyraloidea & Crambidae & Catoptria & myella & Hunedoara & Sibişel & 1999 & August & 28 & 1 \\
\hline 6282 & Pyraloidea & Crambidae & Catoptria & myella & Hunedoara & Sibişel & 2000 & August & 12 & 1 \\
\hline 6282 & Pyraloidea & Crambidae & Catoptria & myella & Hunedoara & Sibişel & 2003 & June & 28 & 1 \\
\hline 6298 & Pyraloidea & Crambidae & Catoptria & pauperellus & Hunedoara & Sibişel & 2002 & July & 16 & 1 \\
\hline 6299 & Pyraloidea & Crambidae & Catoptria & mytilella & Braşov & Tâmpa, Braşov & 1998 & July & 14 & 1 \\
\hline 6350 & Pyraloidea & Crambidae & Thisanotia & chrysonuchella & Braşov & Dealul Lempeş, Sânpetru & 2002 & June & 15 & 5 \\
\hline 6355 & Pyraloidea & Crambidae & Pediasia & luteella & Braşov & Dealul Lempeş, Sânpetru & 2002 & June & 15 & 8 \\
\hline 6355 & Pyraloidea & Crambidae & Pediasia & luteella & Braşov & Roadeş & 2002 & June & 18 & 1 \\
\hline 6355 & Pyraloidea & Crambidae & Pediasia & luteella & Hunedoara & Sibişel & 1999 & August & 8 & 1 \\
\hline 6364 & Pyraloidea & Crambidae & Pediasia & contaminella & Hunedoara & Sibişel & 2001 & July & 10 & 1 \\
\hline 6376 & Pyraloidea & Crambidae & Platytes & cerussella & Braşov & Dealul Lempeş, Sânpetru & 2002 & June & 15 & 3 \\
\hline 6441 & Pyraloidea & Crambidae & Aporodes & floralis & Hunedoara & Sibişel & 2001 & July & 10 & 1 \\
\hline 6499 & Pyraloidea & Crambidae & Evergestis & extimalis & Hunedoara & Beriu & 1984 & June & 20 & 1 \\
\hline 6501 & Pyraloidea & Crambidae & Evergestis & pallidata & Hunedoara & Sibişel & 1999 & August & 11 & 1 \\
\hline 6507 & Pyraloidea & Crambidae & Evergestis & aenealis & Hunedoara & Sibişel & 2002 & July & 29 & 1 \\
\hline 6507 & Pyraloidea & Crambidae & Evergestis & aenealis & Hunedoara & Sibişel & 2003 & July & 10 & 1 \\
\hline 6588 & Pyraloidea & Crambidae & Ecpyrrhorrhoe & rubiginalis & Hunedoara & Sibişel & 1999 & August & 18 & 1 \\
\hline 6588 & Pyraloidea & Crambidae & Ecpyrrhorrhoe & rubiginalis & Hunedoara & Sibişel & 2002 & July & 27 & 1 \\
\hline 6660 & Pyraloidea & Crambidae & Paratalanta & pandalis & Braşov & Pădurea Bogata, Hoghiz & 1980 & June & 22 & 1 \\
\hline 6661 & Pyraloidea & Crambidae & Paratalanta & hyalinalis & Braşov & Poiana Braşov, 1000 m. & 2007 & May & 22 & 1 \\
\hline 6600 & Pyraloidea & Crambidae & Pyrausta & castalis & Hunedoara & Sibişel & 2003 & July & 1 & 1 \\
\hline 6601 & Pyraloidea & Crambidae & Pyrausta & despicata & Braşov & Drăuşeni & 1981 & June & 20 & 1 \\
\hline 6601 & Pyraloidea & Crambidae & Pyrausta & despicata & Braşov & Dealul Lempeş, Sânpetru & 2002 & June & 15 & 2 \\
\hline 6601 & Pyraloidea & Crambidae & Pyrausta & despicata & Braşov & Roadeş & 2002 & June & 18 & 1 \\
\hline 6601 & Pyraloidea & Crambidae & Pyrausta & despicata & Braşov & Pădurea Bogata, Hoghiz & 2007 & May & 27 & 1 \\
\hline 6604 & Pyraloidea & Crambidae & Pyrausta & aurata & Braşov & Tâmpa, Braşov & 1981 & May & 28 & 1 \\
\hline 6604 & Pyraloidea & Crambidae & Pyrausta & aurata & Braşov & Poiana Braşov, 1000 m. & 1981 & May & 28 & 1 \\
\hline 6607 & Pyraloidea & Crambidae & Pyrausta & falcatalis & Braşov & Pădurea Bogata, Hoghiz & 1998 & July & 11 & 1 \\
\hline 6605 & Pyraloidea & Crambidae & Pyrausta & purpuralis & Covasna & Brețcu village & 1982 & July & 23 & 2 \\
\hline 6605 & Pyraloidea & Crambidae & Pyrausta & purpuralis & Hunedoara & Sibişel & 2001 & July & 10 & 1 \\
\hline
\end{tabular}




\begin{tabular}{|c|c|c|c|c|c|c|c|c|c|c|}
\hline $\begin{array}{c}\text { K \& R } \\
\text { LIST NR. }\end{array}$ & SUPERFAMILY & FAMILY & GENUS & SPECIES & COUNTY & LOCALITY & YEAR & MONTH & \multicolumn{2}{|c|}{ DAY NR. } \\
\hline 6605 & Pyraloidea & Crambidae & Pyrausta & purpuralis & Hunedoara & Sibişel & 2003 & June & 27 & 3 \\
\hline 6596 & Pyraloidea & Crambidae & Pyrausta & rectefascialis & Braşov & Tâmpa, Braşov & 1981 & May & 28 & 1 \\
\hline 6624 & Pyraloidea & Crambidae & Sitochroa & verticalis & Braşov & Dealul Lempeş, Sânpetru & 2002 & June & 15 & 8 \\
\hline 6624 & Pyraloidea & Crambidae & Sitochroa & verticalis & Braşov & Dealul Lempeş, Sânpetru & 2007 & May & 29 & 1 \\
\hline 6636 & Pyraloidea & Crambidae & Anania & luctualis & Braşov & Dealul Lempeş, Sânpetru & 1981 & May & 31 & 1 \\
\hline 6655 & Pyraloidea & Crambidae & Anania & verbascalis & Braşov & Dealul Lempeş, Sânpetru & 2007 & May & 27 & 2 \\
\hline 6629 & Pyraloidea & Crambidae & Anania & lancealis & Braşov & Pădurea Bogata, Hoghiz & 1998 & July & 11 & 1 \\
\hline 6629 & Pyraloidea & Crambidae & Anania & lancealis & Hunedoara & Sibişel & 1999 & June & 11 & 1 \\
\hline 6629 & Pyraloidea & Crambidae & Anania & lancealis & Hunedoara & Sibişel & 2003 & June & 24 & 1 \\
\hline 6629 & Pyraloidea & Crambidae & Anania & lancealis & Hunedoara & Sibişel & 2003 & July & 1 & 1 \\
\hline 6631 & Pyraloidea & Crambidae & Anania & coronata & Hunedoara & Sibişel & 1999 & August & 11 & 1 \\
\hline 6632 & Pyraloidea & Crambidae & Anania & stachydalis & Hunedoara & Sibişel & 2000 & August & 21 & 1 \\
\hline 6632 & Pyraloidea & Crambidae & Anania & stachydalis & Hunedoara & Sibişel & 2003 & July & 4 & 1 \\
\hline 6652 & Pyraloidea & Crambidae & Anania & crocealis & Braşov & Tâmpa, Braşov & 1981 & July & 24 & 1 \\
\hline 6652 & Pyraloidea & Crambidae & Anania & crocealis & Braşov & Pădurea Bogata, Hoghiz & 1998 & July & 11 & 1 \\
\hline 6652 & Pyraloidea & Crambidae & Anania & crocealis & Braşov & Drăuşeni & 1998 & July & 2 & 1 \\
\hline 6658 & Pyraloidea & Crambidae & Anania & hortulata & Braşov & Tâmpa, Braşov & 1982 & June & 11 & 1 \\
\hline 6658 & Pyraloidea & Crambidae & Anania & hortulata & Braşov & Pădurea Bogata, Hoghiz & 1980 & June & 22 & 1 \\
\hline 6541 & Pyraloidea & Crambidae & Udea & prunalis & Braşov & Săcele & 1981 & July & 24 & 1 \\
\hline 6557 & Pyraloidea & Crambidae & Udea & olivalis & Braşov & Tâmpa, Braşov & 1981 & July & 24 & 1 \\
\hline 6557 & Pyraloidea & Crambidae & Udea & olivalis & Hunedoara & Sibişel & 2000 & August & 18 & 1 \\
\hline 6557 & Pyraloidea & Crambidae & Udea & olivalis & Hunedoara & Sibişel & 2003 & July & 6 & 1 \\
\hline 6550 & Pyraloidea & Crambidae & Udea & uliginosalis & Braşov & Muntele Piatra Mare, $1500-2000 \mathrm{~m}$. & 1982 & July & 20 & 6 \\
\hline 6547 & Pyraloidea & Crambidae & Udea & alpinalis & Braşov & Muntele Piatra Mare, $1500-2000 \mathrm{~m}$. & 1982 & July & 20 & 4 \\
\hline 6680 & Pyraloidea & Crambidae & Agrotera & nemoralis & Braşov & Dealul Lempeş, Sânpetru & 2007 & May & 29 & 1 \\
\hline 6682 & Pyraloidea & Crambidae & Diasemia & reticularis & Hunedoara & Sibişel & 2003 & July & 2 & 1 \\
\hline 7481 & Drepanoidea & Drepanidae & Thyatira & batis & Hunedoara & Sibişel & 2003 & June & 28 & 1 \\
\hline 7481 & Drepanoidea & Drepanidae & Thyatira & batis & Braşov & Braşov & 2003 & August & 4 & 1 \\
\hline 7483 & Drepanoidea & Drepanidae & Habrosyne & pyritoides & Hunedoara & Sibişel & 2003 & June & 28 & 1 \\
\hline 7490 & Drepanoidea & Drepanidae & Ochropacha & duplaris & Hunedoara & Sibişel & 2001 & July & 12 & 1 \\
\hline 7492 & Drepanoidea & Drepanidae & Cymatophorina & diluta & Hunedoara & Sibişel & 2001 & September & 2 & 1 \\
\hline 7503 & Drepanoidea & Drepanidae & Watsonalla & binaria & Braşov & Dealul Lempeş, Sânpetru & 1979 & July & 14 & 1 \\
\hline
\end{tabular}




\begin{tabular}{|c|c|c|c|c|c|c|c|c|c|c|}
\hline $\begin{array}{c}\text { K \& R } \\
\text { LIST NR. }\end{array}$ & SUPERFAMILY & FAMILY & GENUS & SPECIES & COUNTY & LOCALITY & YEAR & MONTH & \multicolumn{2}{|c|}{ DAY NR. } \\
\hline 7503 & Drepanoidea & Drepanidae & Watsonalla & binaria & Hunedoara & Sibişel & 2001 & August & 31 & 1 \\
\hline 7505 & Drepanoidea & Drepanidae & Watsonalla & cultraria & Braşov & Pietrele lui Solomon, Braşov & 1982 & May & 29 & 2 \\
\hline 7505 & Drepanoidea & Drepanidae & Watsonalla & cultraria & Hunedoara & Sibişel & 1999 & August & 8 & 1 \\
\hline 7505 & Drepanoidea & Drepanidae & Watsonalla & cultraria & Hunedoara & Sibişel & 1999 & August & 25 & 1 \\
\hline 7505 & Drepanoidea & Drepanidae & Watsonalla & cultraria & Hunedoara & Sibişel & 2001 & August & 31 & 1 \\
\hline 7508 & Drepanoidea & Drepanidae & Drepana & falcataria & Covasna & Breţcu village & 1982 & July & 23 & 2 \\
\hline 7508 & Drepanoidea & Drepanidae & Drepana & falcataria & Hunedoara & Sibişel & 2000 & August & 8 & 1 \\
\hline 7508 & Drepanoidea & Drepanidae & Drepana & falcataria & Hunedoara & Sibişel & 2000 & August & 21 & 1 \\
\hline 7508 & Drepanoidea & Drepanidae & Drepana & falcataria & Hunedoara & Sibişel & 2003 & July & 2 & 1 \\
\hline 7512 & Drepanoidea & Drepanidae & Cilix & glaucata & Hunedoara & Sibişel & 2000 & August & 12 & 1 \\
\hline 6731 & Lasiocampoidea & Lasicampidae & Trichiura & crataegi & Hunedoara & Sibişel & 2001 & August & 28 & 1 \\
\hline 6731 & Lasiocampoidea & Lasicampidae & Trichiura & crataegi & Hunedoara & Sibişel & 2001 & September & 5 & 1 \\
\hline 6743 & Lasiocampoidea & Lasicampidae & Malacosoma & neustria & Hunedoara & Sibişel & 1999 & July & 17 & 1 \\
\hline 6743 & Lasiocampoidea & Lasicampidae & Malacosoma & neustria & Hunedoara & Sibişel & 2003 & June & 28 & 1 \\
\hline 6743 & Lasiocampoidea & Lasicampidae & Malacosoma & neustria & Hunedoara & Sibişel & 2003 & July & 22 & 1 \\
\hline 6749 & Lasiocampoidea & Lasicampidae & Lasiocampa & trifolii & Braşov & Homorod & 1969 & August & 16 & 2 \\
\hline 6755 & Lasiocampoidea & Lasicampidae & Macrothylacia & rubi & Covasna & Breţcu village & 1982 & July & 23 & 1 \\
\hline 6773 & Lasiocampoidea & Lasicampidae & Phyllodesma & tremulifolia & Braşov & Braşov, Bartolomeu & 1969 & July & 23 & 1 \\
\hline 6777 & Lasiocampoidea & Lasicampidae & Gastropacha & quercifolia & Braşov & Homorod & 1969 & August & 16 & 1 \\
\hline 6780 & Lasiocampoidea & Lasicampidae & Odonestis & pruni & Hunedoara & Sibişel & 2001 & August & 21 & 1 \\
\hline 6788 & Bombycoidea & Saturniidae & Aglia & tau & Braşov & Dealul Lempeş, Sânpetru & 1982 & May & 29 & 3 \\
\hline 6824 & Bombycoidea & Sphingidae & Laothoe & populi & Braşov & Braşov & 1982 & May & 29 & 1 \\
\hline 6824 & Bombycoidea & Sphingidae & Laothoe & populi & Hunedoara & Sibişel & 1999 & August & 29 & 1 \\
\hline 6828 & Bombycoidea & Sphingidae & Agrius & convolvuli & Braşov & Muntele Postăvar, 2000 m. & 1981 & July & 31 & 1 \\
\hline 6828 & Bombycoidea & Sphingidae & Agrius & convolvuli & Braşov & Braşov & 2002 & September & 25 & 1 \\
\hline 6828 & Bombycoidea & Sphingidae & Agrius & convolvuli & Hunedoara & Sibişel & 2000 & August & 20 & 1 \\
\hline 6830 & Bombycoidea & Sphingidae & Acherontia & atropos & Braşov & Braşov & 1973 & August & 25 & 1 \\
\hline 6834 & Bombycoidea & Sphingidae & Sphinx & pinastri & Braşov & Braşov, Bartolomeu & 1969 & July & 31 & 1 \\
\hline 6839 & Bombycoidea & Sphingidae & Hemaris & tityus & Braşov & Drăuşeni & 1982 & May & 30 & 2 \\
\hline 6840 & Bombycoidea & Sphingidae & Hemaris & fuciformis & Braşov & Şapte Scări, muntele Piatra Mare & 1982 & May & 31 & 1 \\
\hline 6843 & Bombycoidea & Sphingidae & Macroglossum & stellatarum & Braşov & Braşov, Bartolomeu & 1968 & September & 13 & 1 \\
\hline 6843 & Bombycoidea & Sphingidae & Macroglossum & stellatarum & Braşov & Drăuşeni & 1981 & June & 20 & 1 \\
\hline
\end{tabular}




\begin{tabular}{|c|c|c|c|c|c|c|c|c|c|c|}
\hline $\begin{array}{c}\text { K \& R } \\
\text { LIST NR. }\end{array}$ & SUPERFAMILY & FAMILY & GENUS & SPECIES & COUNTY & LOCALITY & YEAR & MONTH & $\overline{\text { DAY }}$ & NR \\
\hline 6853 & Bombycoidea & Sphingidae & Hyles & euphorbiae & Sibiu & Richiria & 1989 & July & 22 & 1 \\
\hline 6860 & Bombycoidea & Sphingidae & Hyles & livornica & Braşov & Tâmpa, Braşov & 1969 & August & 23 & 1 \\
\hline 6862 & Bombycoidea & Sphingidae & Deilephila & elpenor & Hunedoara & Sibişel & 1999 & July & 17 & 1 \\
\hline 6863 & Bombycoidea & Sphingidae & Deilephila & porcellus & Braşov & Homorod & 1969 & August & 16 & 1 \\
\hline 7517 & Geometroidea & Geometridae & Archiearis & parthenias & Braşov & Pietrele lui Solomon, Braşov & 1969 & April & 27 & 1 \\
\hline 7518 & Geometroidea & Geometridae & Boudinotiana & notha & Braşov & Pietrele lui Solomon, Braşov & 1969 & April & 27 & 1 \\
\hline 7522 & Geometroidea & Geometridae & Abraxas & grossulariata & Braşov & Pietrele lui Solomon, Braşov & 1972 & July & 10 & 1 \\
\hline 7524 & Geometroidea & Geometridae & Calospilos & sylvata & Hunedoara & Sibişel & 2003 & June & 28 & 2 \\
\hline 7527 & Geometroidea & Geometridae & Lomaspilis & marginata & Hunedoara & Sibişel & 1999 & August & 8 & 1 \\
\hline 7534 & Geometroidea & Geometridae & Stegania & dilectaria & Hunedoara & Sibişel & 2001 & July & 20 & 1 \\
\hline 7543 & Geometroidea & Geometridae & Macaria & wauaria & Covasna & Breţcu village & 1982 & July & 23 & 4 \\
\hline 7547 & Geometroidea & Geometridae & Chiasmia & clathrata & Braşov & Dealul Lempeş, Sânpetru & 2002 & June & 15 & 2 \\
\hline 7547 & Geometroidea & Geometridae & Chiasmia & clathrata & Braşov & Pădurea Bogata, Hoghiz & 2002 & June & 10 & 1 \\
\hline 7567 & Geometroidea & Geometridae & Macaria & brunneata & Covasna & Breţcu village & 1982 & July & 23 & 1 \\
\hline 7606 & Geometroidea & Geometridae & Plagodis & pulveraria & Braşov & Tâmpa, Braşov & 1981 & May & 29 & 1 \\
\hline 7606 & Geometroidea & Geometridae & Plagodis & pulveraria & Hunedoara & Sibişel & 2002 & July & 29 & 1 \\
\hline 7606 & Geometroidea & Geometridae & Plagodis & pulveraria & Hunedoara & Sibişel & 2003 & August & 15 & 1 \\
\hline 7607 & Geometroidea & Geometridae & Plagodis & dolabraria & Hunedoara & Sibişel & 1999 & Jun & 7 & 1 \\
\hline 7615 & Geometroidea & Geometridae & Epione & repandaria & Covasna & Breţcu village & 1982 & July & 23 & 1 \\
\hline 7616 & Geometroidea & Geometridae & Epione & vespertaria & Covasna & Brețcu village & 1982 & July & 23 & 1 \\
\hline 7618 & Geometroidea & Geometridae & Therapis & flavicaria & Hunedoara & Beriu & 1984 & June & 20 & 1 \\
\hline 7594 & Geometroidea & Geometridae & Cepphis & advenaria & Braşov & Pădurea Bogata, Hoghiz & 2007 & May & 27 & 1 \\
\hline 7620 & Geometroidea & Geometridae & Pseudopanthera & macularia & Braşov & Pădurea Bogata, Hoghiz & 1977 & July & 18 & 1 \\
\hline 7620 & Geometroidea & Geometridae & Pseudopanthera & macularia & Braşov & Pădurea Bogata, Hoghiz & 1979 & May & 17 & 1 \\
\hline 7620 & Geometroidea & Geometridae & Pseudopanthera & macularia & Braşov & Tâmpa, Braşov & 1998 & July & 14 & 1 \\
\hline 7630 & Geometroidea & Geometridae & Apeira & syringaria & Braşov & Dealul Lempeş, Sânpetru & 2003 & August & 5 & 1 \\
\hline 7632 & Geometroidea & Geometridae & Ennomos & autumnaria & Braşov & Pietrele lui Solomon, Braşov & 1969 & August & 23 & 1 \\
\hline 7632 & Geometroidea & Geometridae & Ennomos & autumnaria & Braşov & Braşov, dealul Warthe & 1970 & August & 21 & 1 \\
\hline 7633 & Geometroidea & Geometridae & Ennomos & quercinaria & Braşov & Braşov, dealul Warthe & 1981 & July & 25 & 1 \\
\hline 7633 & Geometroidea & Geometridae & Ennomos & quercinaria & Braşov & Tâmpa, Braşov & 1981 & July & 25 & 1 \\
\hline 7633 & Geometroidea & Geometridae & Ennomos & quercinaria & Braşov & Tâmpa, Braşov & 1982 & July & 28 & 1 \\
\hline 7633 & Geometroidea & Geometridae & Ennomos & quercinaria & Covasna & Breţcu village & 1982 & July & 23 & 2 \\
\hline
\end{tabular}




\begin{tabular}{|c|c|c|c|c|c|c|c|c|c|c|}
\hline $\begin{array}{c}\text { K \& R } \\
\text { LIST NR. }\end{array}$ & SUPERFAMILY & FAMILY & GENUS & SPECIES & COUNTY & LOCALITY & YEAR & MONTH & \multicolumn{2}{|c|}{ DAY NR. } \\
\hline 7641 & Geometroidea & Geometridae & Selenia & dentaria & Covasna & Breţcu village & 1982 & July & 23 & 2 \\
\hline 7641 & Geometroidea & Geometridae & Selenia & dentaria & Hunedoara & Sibişel & 2001 & July & 10 & 2 \\
\hline 7641 & Geometroidea & Geometridae & Selenia & dentaria & Hunedoara & Sibişel & 2002 & July & 10 & 1 \\
\hline 7643 & Geometroidea & Geometridae & Selenia & tetralunaria & Hunedoara & Sibişel & 2001 & July & 10 & 1 \\
\hline 7643 & Geometroidea & Geometridae & Selenia & tetralunaria & Hunedoara & Sibişel & 2003 & July & 3 & 1 \\
\hline 7654 & Geometroidea & Geometridae & Crocallis & elinguaria & Hunedoara & Sibişel & 2001 & July & 10 & 1 \\
\hline 7659 & Geometroidea & Geometridae & Ourapteryx & sambucaria & Braşov & Braşov, Bartolomeu & 1968 & June & 30 & 1 \\
\hline 7659 & Geometroidea & Geometridae & Ourapteryx & sambucaria & Covasna & Brețcu village & 1982 & July & 23 & 1 \\
\hline 7663 & Geometroidea & Geometridae & Colotois & pennaria & Braşov & Braşov, Bartolomeu & 1968 & October & 20 & 1 \\
\hline 7824 & Geometroidea & Geometridae & Cabera & pusaria & Braşov & Tâmpa, Braşov & 1998 & July & 14 & 1 \\
\hline 7824 & Geometroidea & Geometridae & Cabera & pusaria & Braşov & Dealul Bunloc, Săcele & 1991 & July & 15 & 1 \\
\hline 7824 & Geometroidea & Geometridae & Cabera & pusaria & Braşov & Şapte Scări, muntele Piatra Mare & 1981 & July & 24 & 1 \\
\hline 7824 & Geometroidea & Geometridae & Cabera & pusaria & Braşov & Drăuşeni & 1981 & June & 20 & 1 \\
\hline 7826 & Geometroidea & Geometridae & Cabera & exanthemata & Braşov & Şapte Scări, muntele Piatra Mare & 1982 & May & 31 & 1 \\
\hline 7829 & Geometroidea & Geometridae & Lomographa & temerata & Braşov & Tâmpa, Braşov & 1981 & May & 29 & 1 \\
\hline 7829 & Geometroidea & Geometridae & Lomographa & temerata & Hunedoara & Sibişel & 1999 & June & 13 & 1 \\
\hline 7829 & Geometroidea & Geometridae & Lomographa & temerata & Hunedoara & Sibişel & 1999 & July & 21 & 1 \\
\hline 7829 & Geometroidea & Geometridae & Lomographa & temerata & Hunedoara & Sibişel & 2003 & September & 2 & 1 \\
\hline 7836 & Geometroidea & Geometridae & Campaea & margaritata & Braşov & Poiana Braşov, 1000 m. & 1981 & July & 28 & 1 \\
\hline 7836 & Geometroidea & Geometridae & Campaea & margaritata & Braşov & Fundata, 1290 m. & 1982 & July & 19 & 1 \\
\hline 7839 & Geometroidea & Geometridae & Hylaea & fasciaria & Braşov & Muntele Postăvar, 2000 m. & 1968 & July & 2 & 1 \\
\hline 7839 & Geometroidea & Geometridae & Hylaea & fasciaria & Braşov & Muntele Piatra Mare, $1500-2000 \mathrm{~m}$. & 1982 & July & 20 & 2 \\
\hline 7844 & Geometroidea & Geometridae & Pungeleria & capreolaria & Braşov & Poiana Braşov, $1000 \mathrm{~m}$. & 1967 & July & 30 & 1 \\
\hline 7844 & Geometroidea & Geometridae & Pungeleria & capreolaria & Braşov & Muntele Piatra Mare, $1500-2000 \mathrm{~m}$. & 1981 & August & 1 & 1 \\
\hline 7844 & Geometroidea & Geometridae & Pungeleria & capreolaria & Braşov & Muntele Piatra Mare, $1500-2000 \mathrm{~m}$. & 1982 & July & 20 & 3 \\
\hline 7844 & Geometroidea & Geometridae & Pungeleria & capreolaria & Braşov & Dealul Bunloc, Săcele & 1982 & July & 20 & 1 \\
\hline 7844 & Geometroidea & Geometridae & Pungeleria & capreolaria & Covasna & Breţcu village & 1982 & July & 23 & 1 \\
\hline 7862 & Geometroidea & Geometridae & Charissa & ambiguata & Braşov & Muntele Piatra Mare, $1500-2000 \mathrm{~m}$. & 1982 & July & 20 & 3 \\
\hline 7862 & Geometroidea & Geometridae & Charissa & ambiguata & Braşov & Fundata, 1290 m. & 1982 & July & 19 & 1 \\
\hline 7665 & Geometroidea & Geometridae & Angerona & prunaria & Braşov & Braşov, Bartolomeu & 1966 & July & 13 & 2 \\
\hline 7916 & Geometroidea & Geometridae & Siona & lineata & Braşov & Tâmpa, Braşov & 1981 & May & 29 & 1 \\
\hline 7916 & Geometroidea & Geometridae & Siona & lineata & Braşov & Pădurea Bogata, Hoghiz & 1979 & May & 27 & 2 \\
\hline
\end{tabular}




\begin{tabular}{|c|c|c|c|c|c|c|c|c|c|c|}
\hline $\begin{array}{c}\text { K \& R } \\
\text { LIST NR. }\end{array}$ & SUPERFAMILY & FAMILY & GENUS & SPECIES & COUNTY & LOCALITY & YEAR & MONTH & \multicolumn{2}{|c|}{ DAY NR. } \\
\hline 7686 & Geometroidea & Geometridae & Biston & betularia & Hunedoara & Sibişel & 1999 & July & 18 & 1 \\
\hline 7686 & Geometroidea & Geometridae & Biston & betularia & Hunedoara & Sibişel & 1999 & July & 25 & 1 \\
\hline 7686 & Geometroidea & Geometridae & Biston & betularia & Hunedoara & Sibişel & 2001 & June & 10 & 1 \\
\hline 7768 & Geometroidea & Geometridae & Selidosema & plumaria & Braşov & Braşov, Bartolomeu & 1966 & August & 14 & 1 \\
\hline 7768 & Geometroidea & Geometridae & Selidosema & plumaria & Braşov & Tâmpa, Braşov & 1982 & September & 5 & 3 \\
\hline 7777 & Geometroidea & Geometridae & Alcis & repandata & Braşov & Braşov, Bartolomeu & 1969 & August & 6 & 1 \\
\hline 7777 & Geometroidea & Geometridae & Alcis & repandata & Braşov & Şapte Scări, muntele Piatra Mare & 1982 & July & 20 & 1 \\
\hline 7777 & Geometroidea & Geometridae & Alcis & repandata & Braşov & Pădurea Bogata, Hoghiz & 1998 & July & 11 & 1 \\
\hline 7777 & Geometroidea & Geometridae & Alcis & repandata & Covasna & Brețcu village & 1982 & July & 23 & 1 \\
\hline 7777 & Geometroidea & Geometridae & Alcis & repandata & Hunedoara & Sibişel & 1999 & July & 28 & 1 \\
\hline 7777 & Geometroidea & Geometridae & Alcis & repandata & Hunedoara & Sibişel & 2003 & July & 1 & 1 \\
\hline 7778 & Geometroidea & Geometridae & Alcis & deversata & Braşov & Şapte Scări, muntele Piatra Mare & 1981 & August & 1 & 1 \\
\hline 7778 & Geometroidea & Geometridae & Alcis & deversata & Hunedoara & Sibiş̧el & 2000 & August & 12 & 1 \\
\hline 7778 & Geometroidea & Geometridae & Alcis & deversata & Hunedoara & Sibişel & 2002 & July & 17 & 1 \\
\hline 7783 & Geometroidea & Geometridae & Hypomecis & roboraria & Hunedoara & Sibişel & 1999 & July & 17 & 3 \\
\hline 7783 & Geometroidea & Geometridae & Hypomecis & roboraria & Hunedoara & Sibişel & 1999 & July & 22 & 1 \\
\hline 7783 & Geometroidea & Geometridae & Hypomecis & roboraria & Hunedoara & Sibişel & 2003 & June & 22 & 2 \\
\hline 7784 & Geometroidea & Geometridae & Hypomecis & punctinalis & Braşov & Dealul Lempeş, Sânpetru & 2002 & June & 15 & 1 \\
\hline 7784 & Geometroidea & Geometridae & Hypomecis & punctinalis & Covasna & Breţcu village & 1982 & July & 23 & 1 \\
\hline 7794 & Geometroidea & Geometridae & Ascotis & selenaria & Braşov & Braşov, Bartolomeu & 1967 & July & 16 & 1 \\
\hline 7794 & Geometroidea & Geometridae & Ascotis & selenaria & Hunedoara & Sibiş̧el & 2000 & August & 17 & 1 \\
\hline 7796 & Geometroidea & Geometridae & Ectropis & crepuscularia & Braşov & Braşov, Bartolomeu & 1969 & April & 26 & 1 \\
\hline 7796 & Geometroidea & Geometridae & Ectropis & сrepuscularia & Braşov & Tâmpa, Braşov & 1981 & May & 30 & 1 \\
\hline 7796 & Geometroidea & Geometridae & Ectropis & crepuscularia & Braşov & Şapte Scări, muntele Piatra Mare & 1982 & May & 31 & 1 \\
\hline 7796 & Geometroidea & Geometridae & Ectropis & crepuscularia & Hunedoara & Sibişel & 1999 & July & 19 & 1 \\
\hline 7800 & Geometroidea & Geometridae & Parectropis & similaria & Hunedoara & Sibişel & 1999 & June & 3 & 5 \\
\hline 7800 & Geometroidea & Geometridae & Parectropis & similaria & Hunedoara & Sibişel & 1999 & June & 11 & 1 \\
\hline 7804 & Geometroidea & Geometridae & Ematurga & atomaria & Braşov & Tâmpa, Braşov & 1981 & May & 29 & 1 \\
\hline 7804 & Geometroidea & Geometridae & Ematurga & atomaria & Braşov & Săcele & 1981 & July & 24 & 1 \\
\hline 7965 & Geometroidea & Geometridae & Pseudoterpna & pruinata & Braşov & Dealul Lempeş, Sânpetru & 1979 & July & 14 & 1 \\
\hline 7965 & Geometroidea & Geometridae & Pseudoterpna & pruinata & Braşov & Dealul Lempeş, Sânpetru & 1981 & July & 23 & 1 \\
\hline 7965 & Geometroidea & Geometridae & Pseudoterpna & pruinata & Hunedoara & Sibişel & 2002 & July & 16 & 1 \\
\hline
\end{tabular}




\begin{tabular}{|c|c|c|c|c|c|c|c|c|c|c|}
\hline $\begin{array}{c}\text { K \& R } \\
\text { LIST NR. }\end{array}$ & SUPERFAMILY & FAMILY & GENUS & SPECIES & COUNTY & LOCALITY & YEAR & MONTH & DAY & NR. \\
\hline 7969 & Geometroidea & Geometridae & Geometra & papilionaria & Hunedoara & Sibişel & 2003 & July & 2 & 1 \\
\hline 7975 & Geometroidea & Geometridae & Thetidia & smaragdaria & Braşov & Drăuşeni & 1981 & June & 20 & 1 \\
\hline 7982 & Geometroidea & Geometridae & Chlorissa & viridata & Braşov & Dealul Lempeş, Sânpetru & 1982 & May & 29 & 1 \\
\hline 7982 & Geometroidea & Geometridae & Chlorissa & viridata & Hunedoara & Sibişel & 1999 & July & 20 & 1 \\
\hline 7982 & Geometroidea & Geometridae & Chlorissa & viridata & Hunedoara & Sibişel & 2000 & August & 9 & 1 \\
\hline 7982 & Geometroidea & Geometridae & Chlorissa & viridata & Hunedoara & Sibişel & 2003 & July & 1 & 2 \\
\hline 7983 & Geometroidea & Geometridae & Chlorissa & cloraria & Hunedoara & Sibişel & 1999 & July & 22 & 1 \\
\hline 8100 & Geometroidea & Geometridae & Idaea & serpentata & Braşov & Drăuşeni & 1981 & June & 20 & 1 \\
\hline 8100 & Geometroidea & Geometridae & Idaea & serpentata & Braşov & Drăuşeni & 1982 & May & 30 & 3 \\
\hline 8100 & Geometroidea & Geometridae & Idaea & serpentata & Braşov & Fundata, $1290 \mathrm{~m}$. & 1998 & July & 13 & 2 \\
\hline 8100 & Geometroidea & Geometridae & Idaea & serpentata & Braşov & Pădurea Bogata, Hoghiz & 2007 & May & 27 & 1 \\
\hline 8100 & Geometroidea & Geometridae & Idaea & serpentata & Covasna & Vâlcele & 1981 & June & 19 & 1 \\
\hline 8102 & Geometroidea & Geometridae & Idaea & aureolaria & Braşov & Tâmpa, Braşov & 1991 & July & 17 & 4 \\
\hline 8102 & Geometroidea & Geometridae & Idaea & aureolaria & Braşov & Tâmpa, Braşov & 1998 & July & 14 & 1 \\
\hline 8132 & Geometroidea & Geometridae & Idaea & biselata & Braşov & Săcele & 1981 & July & 24 & 1 \\
\hline 8132 & Geometroidea & Geometridae & Idaea & biselata & Hunedoara & Sibişel & 2001 & July & 20 & 1 \\
\hline 8134 & Geometroidea & Geometridae & Idaea & inquinata & Braşov & Tâmpa, Braşov & 1998 & July & 14 & 1 \\
\hline 8140 & Geometroidea & Geometridae & Idaea & humiliata & Braşov & Tâmpa, Braşov & 1998 & July & 14 & 2 \\
\hline 8140 & Geometroidea & Geometridae & Idaea & humiliata & Braşov & Tâmpa, Braşov & 2002 & June & 17 & 1 \\
\hline 8140 & Geometroidea & Geometridae & Idaea & humiliata & Braşov & Drăuşeni & 1998 & July & 2 & 1 \\
\hline 8155 & Geometroidea & Geometridae & Idaea & seriata & Covasna & Brețcu village & 1982 & July & 23 & 1 \\
\hline 8161 & Geometroidea & Geometridae & Idaea & dimidiata & Covasna & Breţcu village & 1982 & July & 23 & 1 \\
\hline 8168 & Geometroidea & Geometridae & Idaea & pallidata & Braşov & Pădurea Bogata, Hoghiz & 2007 & May & 27 & 1 \\
\hline 8036 & Geometroidea & Geometridae & Scopula & immorata & Hunedoara & Sibişel & 2000 & August & 8 & 1 \\
\hline 8036 & Geometroidea & Geometridae & Scopula & immorata & Hunedoara & Sibişel & 2001 & September & 1 & 1 \\
\hline 8036 & Geometroidea & Geometridae & Scopula & immorata & Hunedoara & Sibişel & 2002 & July & 29 & 1 \\
\hline 8037 & Geometroidea & Geometridae & Scopula & tessellaria & Braşov & Dealul Lempeş, Sânpetru & 2002 & June & 15 & 1 \\
\hline 8043 & Geometroidea & Geometridae & Scopula & virgulata & Braşov & Dealul Lempeş, Sânpetru & 2002 & June & 15 & 8 \\
\hline 8045 & Geometroidea & Geometridae & Scopula & ornata & Braşov & Dealul Lempeş, Sânpetru & 1979 & July & 29 & 1 \\
\hline 8045 & Geometroidea & Geometridae & Scopula & ornata & Braşov & Dealul Lempeş, Sânpetru & 1982 & May & 25 & 1 \\
\hline 8045 & Geometroidea & Geometridae & Scopula & ornata & Braşov & Dealul Lempeş, Sânpetru & 1982 & May & 29 & 1 \\
\hline 8045 & Geometroidea & Geometridae & Scopula & ornata & Braşov & Dealul Lempeş, Sânpetru & 2002 & June & 17 & 1 \\
\hline
\end{tabular}




\begin{tabular}{|c|c|c|c|c|c|c|c|c|c|c|}
\hline $\begin{array}{c}\text { K \& R } \\
\text { LIST NR. }\end{array}$ & SUPERFAMILY & FAMILY & GENUS & SPECIES & COUNTY & LOCALITY & YEAR & MONTH & \multicolumn{2}{|c|}{ DAY NR } \\
\hline 8054 & Geometroidea & Geometridae & Scopula & rubiginata & Braşov & Drăuşeni & 1981 & June & 20 & 1 \\
\hline 8064 & Geometroidea & Geometridae & Scopula & immutata & Braşov & Drăuşeni & 1980 & June & 22 & 2 \\
\hline 8069 & Geometroidea & Geometridae & Scopula & floslactata & Braşov & Cheile Râşnoavei, Râşnov & 1980 & June & 21 & 1 \\
\hline 8069 & Geometroidea & Geometridae & Scopula & floslactata & Braşov & Braşov, dealul Warthe & 1982 & June & 14 & 2 \\
\hline 8205 & Geometroidea & Geometridae & Rhodostrophia & vibicaria & Braşov & Tâmpa, Braşov & 1981 & July & 25 & 1 \\
\hline 8205 & Geometroidea & Geometridae & Rhodostrophia & vibicaria & Braşov & Tâmpa, Braşov & 1998 & July & 14 & 2 \\
\hline 8205 & Geometroidea & Geometridae & Rhodostrophia & vibicaria & Braşov & Fundata, $1290 \mathrm{~m}$. & 1982 & July & 19 & 1 \\
\hline 8205 & Geometroidea & Geometridae & Rhodostrophia & vibicaria & Covasna & Breţcu village & 1982 & July & 23 & 1 \\
\hline 8014 & Geometroidea & Geometridae & Cyclophora & annularia & Hunedoara & Sibişel & 1999 & June & 13 & 1 \\
\hline 8024 & Geometroidea & Geometridae & Cyclophora & linearia & Braşov & Tâmpa, Braşov & 1982 & June & 14 & 1 \\
\hline 8024 & Geometroidea & Geometridae & Cyclophora & linearia & Hunedoara & Sibişel & 1999 & August & 8 & 1 \\
\hline 8240 & Geometroidea & Geometridae & Scotopteryx & mucronata & Hunedoara & Sibişel & 2001 & July & 10 & 1 \\
\hline 8236 & Geometroidea & Geometridae & Scotopteryx & bipunctaria & Braşov & Drăuşeni & 1978 & July & 28 & 1 \\
\hline 8236 & Geometroidea & Geometridae & Scotopteryx & bipunctaria & Hunedoara & Sibiş̧el & 2001 & July & 14 & 1 \\
\hline 8229 & Geometroidea & Geometridae & Scotopteryx & moeniata & Hunedoara & Sibişel & 2000 & August & 14 & 1 \\
\hline 8229 & Geometroidea & Geometridae & Scotopteryx & moeniata & Hunedoara & Sibişel & 2001 & July & 10 & 1 \\
\hline 8239 & Geometroidea & Geometridae & Scotopteryx & chenopodiata & Braşov & Dealul Lempeş, Sânpetru & 1981 & July & 23 & 1 \\
\hline 8239 & Geometroidea & Geometridae & Scotopteryx & chenopodiata & Braşov & Şapte Scări, muntele Piatra Mare & 1981 & July & 24 & 1 \\
\hline 8239 & Geometroidea & Geometridae & Scotopteryx & chenopodiata & Braşov & Şapte Scări, muntele Piatra Mare & 1982 & July & 20 & 1 \\
\hline 8239 & Geometroidea & Geometridae & Scotopteryx & chenopodiata & Braşov & Tâmpa, Braşov & 1998 & July & 14 & 1 \\
\hline 8239 & Geometroidea & Geometridae & Scotopteryx & chenopodiata & Braşov & Fundata, $1290 \mathrm{~m}$. & 1982 & July & 19 & 1 \\
\hline 8239 & Geometroidea & Geometridae & Scotopteryx & chenopodiata & Covasna & Breţcu village & 1982 & July & 23 & 3 \\
\hline 8239 & Geometroidea & Geometridae & Scotopteryx & chenopodiata & Covasna & Vâlcele & 1981 & June & 19 & 1 \\
\hline 8239 & Geometroidea & Geometridae & Scotopteryx & chenopodiata & Hunedoara & Sibişel & 2002 & July & 16 & 1 \\
\hline 8256 & Geometroidea & Geometridae & Xanthorhoe & fluctuata & Braşov & Homorod & 1981 & June & 20 & 1 \\
\hline 8248 & Geometroidea & Geometridae & Xanthorhoe & biriviata & Braşov & Cheile Râşnoavei, Râşnov & 1982 & June & 12 & 1 \\
\hline 8248 & Geometroidea & Geometridae & Xanthorhoe & biriviata & Braşov & Şapte Scări, muntele Piatra Mare & 1982 & May & 31 & 1 \\
\hline 8248 & Geometroidea & Geometridae & Xanthorhoe & biriviata & Covasna & Breţcu village & 1982 & July & 23 & 1 \\
\hline 8252 & Geometroidea & Geometridae & Xanthorhoe & spadicearia & Braşov & Muntele Postăvar, 2000 m. & 1981 & July & 24 & 1 \\
\hline 8252 & Geometroidea & Geometridae & Xanthorhoe & spadicearia & Braşov & Cheile Râşnoavei, Râşnov & 1980 & June & 21 & 1 \\
\hline 8252 & Geometroidea & Geometridae & Xanthorhoe & spadicearia & Braşov & Şapte Scări, muntele Piatra Mare & 1982 & May & 31 & 2 \\
\hline 8252 & Geometroidea & Geometridae & Xanthorhoe & spadicearia & Braşov & Braşov, dealul Warthe & 1981 & May & 30 & 1 \\
\hline
\end{tabular}




\begin{tabular}{|c|c|c|c|c|c|c|c|c|c|c|}
\hline $\begin{array}{c}\text { K \& R } \\
\text { LIST NR. }\end{array}$ & SUPERFAMILY & FAMILY & GENUS & SPECIES & COUNTY & LOCALITY & YEAR & MONTH & \multicolumn{2}{|c|}{ DAY NR. } \\
\hline 8252 & Geometroidea & Geometridae & Xanthorhoe & spadicearia & Braşov & Dealul Bunloc, Săcele & 1981 & July & 26 & 1 \\
\hline 8253 & Geometroidea & Geometridae & Xanthorhoe & ferrugata & Braşov & Tâmpa, Braşov & 1981 & May & 30 & 1 \\
\hline 8253 & Geometroidea & Geometridae & Xanthorhoe & ferrugata & Covasna & Brețcu village & 1982 & July & 23 & 1 \\
\hline 8253 & Geometroidea & Geometridae & Xanthorhoe & ferrugata & Hunedoara & Sibişel & 2003 & July & 1 & 1 \\
\hline 8249 & Geometroidea & Geometridae & Xanthorhoe & designata & Hunedoara & Sibişel & 1999 & June & 13 & 1 \\
\hline 8255 & Geometroidea & Geometridae & Xanthorhoe & montanata & Braşov & Cheile Râşnoavei, Râşnov & 1981 & May & 30 & 2 \\
\hline 8255 & Geometroidea & Geometridae & Xanthorhoe & montanata & Hunedoara & Sibişel & 1999 & June & 24 & 1 \\
\hline 8254 & Geometroidea & Geometridae & Xanthorhoe & quadrifasciata & Covasna & Breţcu village & 1982 & July & 23 & 2 \\
\hline 8254 & Geometroidea & Geometridae & Xanthorhoe & quadrifasciata & Hunedoara & Sibişel & 2003 & July & 1 & 1 \\
\hline 8269 & Geometroidea & Geometridae & Catarhoe & cuculata & Covasna & Vâlcele & 2003 & August & 5 & 1 \\
\hline 8289 & Geometroidea & Geometridae & Camptogramma & bilineata & Braşov & Dealul Lempeş, Sânpetru & 1979 & July & 29 & 1 \\
\hline 8289 & Geometroidea & Geometridae & Camptogramma & bilineata & Braşov & Pădurea Bogata, Hoghiz & 1998 & July & 11 & 1 \\
\hline 8440 & Geometroidea & Geometridae & Camptogramma & scripturata & Braşov & Fundata, 1290 m. & 1998 & July & 13 & 1 \\
\hline 8440 & Geometroidea & Geometridae & Camptogramma & scripturata & Hunedoara & Sibişel & 2002 & July & 16 & 1 \\
\hline 8274 & Geometroidea & Geometridae & Epirrhoe & tristata & Braşov & Dealul Lempeş, Sânpetru & 1982 & May & 29 & 3 \\
\hline 8274 & Geometroidea & Geometridae & Epirrhoe & tristata & Braşov & Muntele Postăvar, 2000 m. & 1981 & May & 30 & 1 \\
\hline 8274 & Geometroidea & Geometridae & Epirrhoe & tristata & Braşov & Poiana Braşov, 1000 m. & 2007 & May & 22 & 1 \\
\hline 8274 & Geometroidea & Geometridae & Epirrhoe & tristata & Braşov & Şapte Scări, muntele Piatra Mare & 1981 & May & 28 & 1 \\
\hline 8274 & Geometroidea & Geometridae & Epirrhoe & tristata & Braşov & Şapte Scări, muntele Piatra Mare & 2004 & April & 21 & 1 \\
\hline 8277 & Geometroidea & Geometridae & Epirrhoe & rivata & Hunedoara & Sibişel & 1999 & June & 24 & 1 \\
\hline 8436 & Geometroidea & Geometridae & Euphyia & unangulata & Hunedoara & Sibişel & 1999 & August & 16 & 2 \\
\hline 8312 & Geometroidea & Geometridae & Mesoleuca & albicillata & Hunedoara & Sibişel & 2001 & July & 10 & 1 \\
\hline 8314 & Geometroidea & Geometridae & Pelurga & comitata & Covasna & Breţcu village & 1982 & July & 23 & 1 \\
\hline 8302 & Geometroidea & Geometridae & Entephria & caesiata & Hunedoara & Sibişel & 2002 & July & 19 & 1 \\
\hline 8417 & Geometroidea & Geometridae & Spargania & luctuata & Braşov & Dealul Lempeş, Sânpetru & 1979 & July & 29 & 2 \\
\hline 8417 & Geometroidea & Geometridae & Spargania & luctuata & Braşov & Şapte Scări, muntele Piatra Mare & 1982 & May & 31 & 1 \\
\hline 8417 & Geometroidea & Geometridae & Spargania & luctuata & Braşov & Tâmpa, Braşov & 1998 & July & 14 & 1 \\
\hline 8391 & Geometroidea & Geometridae & Hydriomena & furcata & Braşov & Muntele Postăvar, 2000 m. & 1981 & July & 24 & 1 \\
\hline 8391 & Geometroidea & Geometridae & Hydriomena & furcata & Braşov & Pădurea Bogata, Hoghiz & 1998 & July & 11 & 1 \\
\hline 8391 & Geometroidea & Geometridae & Hydriomena & furcata & Covasna & Brețcu village & 1982 & July & 23 & 1 \\
\hline 8357 & Geometroidea & Geometridae & Thera & variata & Braşov & Muntele Piatra Mare, $1500-2000 \mathrm{~m}$. & 1982 & July & 20 & 4 \\
\hline 8357 & Geometroidea & Geometridae & Thera & variata & Hunedoara & Sibişel & 1999 & June & 6 & 1 \\
\hline
\end{tabular}




\begin{tabular}{|c|c|c|c|c|c|c|c|c|c|c|}
\hline $\begin{array}{c}\text { K \& R } \\
\text { LIST NR. }\end{array}$ & SUPERFAMILY & FAMILY & GENUS & SPECIES & COUNTY & LOCALITY & YEAR & MONTH & \multicolumn{2}{|c|}{ DAY NR } \\
\hline 8357 & Geometroidea & Geometridae & Thera & variata & Hunedoara & Sibişel & 2003 & July & 2 & 1 \\
\hline 8352 & Geometroidea & Geometridae & Plemyria & rubiginata & Covasna & Brețcu village & 1982 & July & 23 & 1 \\
\hline 8350 & Geometroidea & Geometridae & Cidaria & fulvata & Hunedoara & Sibişel & 1999 & June & 18 & 1 \\
\hline 8319 & Geometroidea & Geometridae & Cosmorhoe & ocellata & Braşov & Şapte Scări, muntele Piatra Mare & 1981 & May & 28 & 1 \\
\hline 8319 & Geometroidea & Geometridae & Cosmorhoe & ocellata & Hunedoara & Sibişel & 1999 & August & 3 & 1 \\
\hline 8319 & Geometroidea & Geometridae & Cosmorhoe & ocellata & Hunedoara & Sibişel & 2000 & August & 12 & 1 \\
\hline 8319 & Geometroidea & Geometridae & Cosmorhoe & ocellata & Hunedoara & Sibişel & 2001 & July & 10 & 1 \\
\hline 8366 & Geometroidea & Geometridae & Eustroma & reticulata & Covasna & Brețcu village & 1982 & July & 23 & 2 \\
\hline 8332 & Geometroidea & Geometridae & Eulithis & populata & Covasna & Brețcu village & 1982 & July & 23 & 1 \\
\hline 8335 & Geometroidea & Geometridae & Gandaritis & pyraliata & Braşov & Teliu, 1500 m., munţii Întorsurii & 1978 & July & 30 & 1 \\
\hline 8338 & Geometroidea & Geometridae & Ecliptopera & silaceata & Hunedoara & Sibişel & 1999 & August & 18 & 1 \\
\hline 8338 & Geometroidea & Geometridae & Ecliptopera & silaceata & Hunedoara & Sibişel & 2002 & July & 27 & 1 \\
\hline 8338 & Geometroidea & Geometridae & Ecliptopera & silaceata & Hunedoara & Sibişel & 2003 & August & 17 & 1 \\
\hline 8341 & Geometroidea & Geometridae & Chloroclysta & siterata & Braşov & Braşov & 2003 & September & 14 & 2 \\
\hline 8348 & Geometroidea & Geometridae & Dysstroma & truncata & Braşov & Tâmpa, Braşov & 1969 & July & 12 & 1 \\
\hline 8348 & Geometroidea & Geometridae & Dysstroma & truncata & Braşov & Braşov & 2003 & September & 14 & 1 \\
\hline 8348 & Geometroidea & Geometridae & Dysstroma & truncata & Hunedoara & Sibişel & 1999 & June & 8 & 1 \\
\hline 8348 & Geometroidea & Geometridae & Dysstroma & truncata & Hunedoara & Sibişel & 2002 & July & 30 & 1 \\
\hline 8385 & Geometroidea & Geometridae & Colostygia & pectinataria & Covasna & Vâlcele & 1981 & June & 19 & 1 \\
\hline 8385 & Geometroidea & Geometridae & Colostygia & pectinataria & Hunedoara & Sibişel & 1999 & August & 18 & 2 \\
\hline 8378 & Geometroidea & Geometridae & Colostygia & turbata & Braşov & Muntele Piatra Mare, $1500-2000 \mathrm{~m}$. & 1982 & July & 20 & 1 \\
\hline 8321 & Geometroidea & Geometridae & Coenotephria & salicata & Braşov & Tâmpa, Braşov & 1981 & May & 29 & 2 \\
\hline 8316 & Geometroidea & Geometridae & Lampropteryx & suffumata & Braşov & Dealul Bunloc, Săcele & 1981 & April & 24 & 1 \\
\hline 8316 & Geometroidea & Geometridae & Lampropteryx & suffumata & Braşov & Muntele Postăvar, 2000 m. & 1981 & May & 30 & 1 \\
\hline 8316 & Geometroidea & Geometridae & Lampropteryx & suffumata & Braşov & Şapte Scări, muntele Piatra Mare & 1982 & May & 31 & 1 \\
\hline 8442 & Geometroidea & Geometridae & Epirrita & dilutata & Braşov & Tâmpa, Braşov & 1978 & October & 16 & 1 \\
\hline 8663 & Geometroidea & Geometridae & Minoa & murinata & Braşov & Tâmpa, Braşov & 1981 & May & 30 & 1 \\
\hline 8663 & Geometroidea & Geometridae & Minoa & murinata & Braşov & Fundata, $1290 \mathrm{~m}$. & 1982 & July & 19 & 2 \\
\hline 8656 & Geometroidea & Geometridae & Asthena & albulata & Braşov & Tâmpa, Braşov & 1982 & June & 12 & 1 \\
\hline 8656 & Geometroidea & Geometridae & Asthena & albulata & Braşov & Braşov, dealul Warthe & 1982 & June & 14 & 1 \\
\hline 8656 & Geometroidea & Geometridae & Asthena & albulata & Hunedoara & Sibişel & 2001 & July & 10 & 3 \\
\hline 8660 & Geometroidea & Geometridae & Hydrelia & flammeolaria & Hunedoara & Sibişel & 2003 & July & 6 & 1 \\
\hline
\end{tabular}




\begin{tabular}{|c|c|c|c|c|c|c|c|c|c|c|}
\hline $\begin{array}{c}\text { K \& R } \\
\text { LIST NR. }\end{array}$ & SUPERFAMILY & FAMILY & GENUS & SPECIES & COUNTY & LOCALITY & YEAR & MONTH & \multicolumn{2}{|c|}{ DAY NR. } \\
\hline 8660 & Geometroidea & Geometridae & Hydrelia & flammeolaria & Hunedoara & Sibişel & 2001 & July & 10 & 2 \\
\hline 8650 & Geometroidea & Geometridae & Venusia & blomeri & Hunedoara & Sibişel & 1999 & June & 7 & 1 \\
\hline 8411 & Geometroidea & Geometridae & Melanthia & procellata & Braşov & Tâmpa, Braşov & 1969 & June & 1 & 1 \\
\hline 8411 & Geometroidea & Geometridae & Melanthia & procellata & Hunedoara & Sibişel & 1999 & August & 16 & 1 \\
\hline 8411 & Geometroidea & Geometridae & Melanthia & procellata & Hunedoara & Sibişel & 2001 & July & 10 & 1 \\
\hline 8631 & Geometroidea & Geometridae & Odezia & atrata & Braşov & Dealul Bunloc, Săcele & 1969 & July & 18 & 1 \\
\hline 8624 & Geometroidea & Geometridae & Aplocera & praeformata & Braşov & Braşov, Bartolomeu & 1967 & June & 25 & 1 \\
\hline 8624 & Geometroidea & Geometridae & Aplocera & praeformata & Braşov & Tâmpa, Braşov & 1982 & July & 18 & 1 \\
\hline 8624 & Geometroidea & Geometridae & Aplocera & praeformata & Hunedoara & Sibişel & 2002 & July & 12 & 1 \\
\hline 8665 & Geometroidea & Geometridae & Lobophora & halterata & Braşov & Pădurea Bogata, Hoghiz & 2004 & April & 23 & 1 \\
\hline 8455 & Geometroidea & Geometridae & Perizoma & affinitata & Hunedoara & Sibişel & 2003 & July & 9 & 1 \\
\hline 8456 & Geometroidea & Geometridae & Perizoma & alchemillata & Covasna & Breţcu village & 1982 & July & 23 & 3 \\
\hline 8456 & Geometroidea & Geometridae & Perizoma & alchemillata & Braşov & Tâmpa, Braşov & 1991 & July & 17 & 1 \\
\hline 8456 & Geometroidea & Geometridae & Perizoma & alchemillata & Hunedoara & Sibişel & 1999 & July & 30 & 1 \\
\hline 8456 & Geometroidea & Geometridae & Perizoma & alchemillata & Hunedoara & Sibişel & 2000 & August & 8 & 1 \\
\hline 8456 & Geometroidea & Geometridae & Perizoma & alchemillata & Hunedoara & Sibişel & 2003 & July & 10 & 1 \\
\hline 8458 & Geometroidea & Geometridae & Perizoma & lugdunaria & Hunedoara & Sibiş̧el & 2003 & July & 2 & 1 \\
\hline 8461 & Geometroidea & Geometridae & Perizoma & minorata & Braşov & Muntele Piatra Mare, $1500-2000 \mathrm{~m}$. & 1982 & July & 20 & 1 \\
\hline 8463 & Geometroidea & Geometridae & Perizoma & albulata & Braşov & Poiana Braşov, 1000 m. & 1981 & May & 30 & 3 \\
\hline 8463 & Geometroidea & Geometridae & Perizoma & albulata & Braşov & Drăuşeni & 1982 & May & 30 & 3 \\
\hline 8463 & Geometroidea & Geometridae & Perizoma & albulata & Hunedoara & Sibişel & 2003 & June & 23 & 1 \\
\hline 8601 & Geometroidea & Geometridae & Chloroclystis & $v$-ata & Hunedoara & Sibişel & 2003 & June & 20 & 1 \\
\hline 8601 & Geometroidea & Geometridae & Chloroclystis & $v$-ata & Hunedoara & Sibişel & 2003 & June & 25 & 3 \\
\hline 8601 & Geometroidea & Geometridae & Chloroclystis & $v$-ata & Hunedoara & Sibişel & 2003 & July & 8 & 1 \\
\hline 8604 & Geometroidea & Geometridae & Pasiphila & chloerata & Braşov & Tâmpa, Braşov & 1982 & June & 11 & 1 \\
\hline 8604 & Geometroidea & Geometridae & Pasiphila & chloerata & Covasna & Breţcu village & 1982 & July & 23 & 1 \\
\hline 8604 & Geometroidea & Geometridae & Pasiphila & chloerata & Hunedoara & Sibişel & 1999 & August & 8 & 1 \\
\hline 8479 & Geometroidea & Geometridae & Eupithecia & plumbeolata & Braşov & Braşov, dealul Warthe & 1981 & May & 30 & 2 \\
\hline 8557 & Geometroidea & Geometridae & Eupithecia & sinuosaria & Hunedoara & Sibişel & 2001 & July & 10 & 1 \\
\hline 8490 & Geometroidea & Geometridae & Eupithecia & irriguata & Braşov & Poiana Braşov, 1000 m. & 2007 & May & 22 & 1 \\
\hline 8509 & Geometroidea & Geometridae & Eupithecia & centaureata & Braşov & Tâmpa, Braşov & 1981 & May & 31 & 1 \\
\hline 8509 & Geometroidea & Geometridae & Eupithecia & centaureata & Covasna & Brețcu village & 1982 & July & 23 & 3 \\
\hline
\end{tabular}




\begin{tabular}{|c|c|c|c|c|c|c|c|c|c|c|}
\hline $\begin{array}{c}\text { K \& R } \\
\text { LIST NR. }\end{array}$ & SUPERFAMILY & FAMILY & GENUS & SPECIES & COUNTY & LOCALITY & YEAR & MONTH & \multicolumn{2}{|c|}{ DAY NR. } \\
\hline 8536 & Geometroidea & Geometridae & Eupithecia & denotata & Braşov & Poiana Braşov, 1000 m. & 1982 & August & 13 & 1 \\
\hline 8716 & Noctuoidea & Notodontidae & Notodonta & dromedarius & Covasna & Brețcu village & 1982 & July & 23 & 1 \\
\hline 8718 & Noctuoidea & Notodontidae & Notodonta & tritophus & Covasna & Breţcu village & 1982 & July & 23 & 1 \\
\hline 8727 & Noctuoidea & Notodontidae & Pheosia & tremula & Braşov & Drăuşeni & 1969 & August & 16 & 1 \\
\hline 8732 & Noctuoidea & Notodontidae & Pterostoma & palpina & Hunedoara & Sibişel & 1999 & July & 17 & 1 \\
\hline 8734 & Noctuoidea & Notodontidae & Ptilophora & plumigera & Braşov & Tâmpa, Braşov & 1982 & December & 20 & 1 \\
\hline 8738 & Noctuoidea & Notodontidae & Ptilodon & capucina & Braşov & Vlădeni & 1971 & July & 27 & 1 \\
\hline 8738 & Noctuoidea & Notodontidae & Ptilodon & capucina & Hunedoara & Sibişel & 2003 & June & 27 & 1 \\
\hline 8738 & Noctuoidea & Notodontidae & Ptilodon & capucina & Hunedoara & Sibişel & 2003 & July & 2 & 1 \\
\hline 8750 & Noctuoidea & Notodontidae & Phalera & bucephala & Braşov & Braşov, Bartolomeu & 1969 & July & 19 & 1 \\
\hline 8750 & Noctuoidea & Notodontidae & Phalera & bucephala & Covasna & Brețcu village & 1982 & July & 23 & 1 \\
\hline 8762 & Noctuoidea & Notodontidae & Spatalia & argentina & Hunedoara & Sibişel & 2003 & June & 29 & 1 \\
\hline 9008 & Noctuoidea & Noctuidae & Rivula & sericealis & Braşov & Braşov & 1982 & June & 11 & 1 \\
\hline 8868 & Noctuoidea & Noctuidae & Schrankia & taenialis & Braşov & Şapte Scări, muntele Piatra Mare & 1982 & July & 20 & 1 \\
\hline 8835 & Noctuoidea & Noctuidae & Idia & calvaria & Covasna & Breţcu village & 1982 & July & 23 & 1 \\
\hline 8835 & Noctuoidea & Noctuidae & Idia & calvaria & Hunedoara & Sibişel & 2003 & July & 1 & 1 \\
\hline 8839 & Noctuoidea & Noctuidae & Paracolax & tristalis & Hunedoara & Sibişel & 2000 & August & 18 & 1 \\
\hline 8858 & Noctuoidea & Noctuidae & Herminia & tarsipennalis & Hunedoara & Sibişel & 2002 & August & 15 & 1 \\
\hline 8846 & Noctuoidea & Noctuidae & Herminia & grisealis & Hunedoara & Sibişel & 1999 & August & 15 & 2 \\
\hline 8846 & Noctuoidea & Noctuidae & Herminia & grisealis & Hunedoara & Sibişel & 2001 & July & 10 & 1 \\
\hline 8846 & Noctuoidea & Noctuidae & Herminia & grisealis & Hunedoara & Sibişel & 2002 & July & 27 & 1 \\
\hline 8849 & Noctuoidea & Noctuidae & Polypogon & tentacularia & Braşov & Dealul Lempeş, Sânpetru & 1979 & July & 14 & 2 \\
\hline 8849 & Noctuoidea & Noctuidae & Polypogon & tentacularia & Braşov & Drăuşeni & 1981 & June & 20 & 2 \\
\hline 8849 & Noctuoidea & Noctuidae & Polypogon & tentacularia & Braşov & Pădurea Bogata, Hoghiz & 1998 & July & 11 & 1 \\
\hline 8850 & Noctuoidea & Noctuidae & Polypogon & gryphalis & Braşov & Roadeş & 2002 & June & 18 & 1 \\
\hline 8994 & Noctuoidea & Noctuidae & Hypena & proboscidalis & Braşov & Tâmpa, Braşov & 1982 & July & 18 & 1 \\
\hline 8994 & Noctuoidea & Noctuidae & Hypena & proboscidalis & Braşov & Şapte Scări, muntele Piatra Mare & 1981 & August & 1 & 1 \\
\hline 8994 & Noctuoidea & Noctuidae & Hypena & proboscidalis & Braşov & Pădurea Bogata, Hoghiz & 1998 & July & 11 & 1 \\
\hline 8994 & Noctuoidea & Noctuidae & Hypena & proboscidalis & Hunedoara & Sibişel & 1999 & August & 12 & 1 \\
\hline 8994 & Noctuoidea & Noctuidae & Hypena & proboscidalis & Hunedoara & Sibişel & 2001 & July & 19 & 1 \\
\hline 8995 & Noctuoidea & Noctuidae & Hypena & rostralis & Hunedoara & Sibişel & 2003 & July & 9 & 1 \\
\hline 9006 & Noctuoidea & Noctuidae & Phytometra & viridaria & Hunedoara & Sibişel & 2002 & July & 25 & 1 \\
\hline
\end{tabular}




\begin{tabular}{|c|c|c|c|c|c|c|c|c|c|c|}
\hline $\begin{array}{c}\text { K \& R } \\
\text { LIST NR. }\end{array}$ & SUPERFAMILY & FAMILY & GENUS & SPECIES & COUNTY & LOCALITY & YEAR & MONTH & DAY & NR. \\
\hline 9006 & Noctuoidea & Noctuidae & Phytometra & viridaria & Hunedoara & Sibişel & 2003 & June & 25 & 1 \\
\hline 9018 & Noctuoidea & Noctuidae & Colobochyla & salicalis & Hunedoara & Sibişel & 2001 & August & 16 & 1 \\
\hline 8975 & Noctuoidea & Noctuidae & Laspeyria & flexula & Covasna & Brețcu village & 1982 & July & 23 & 3 \\
\hline 8984 & Noctuoidea & Noctuidae & Scoliopteyx & libatrix & Braşov & Braşov, Bartolomeu & 1968 & September & 16 & 1 \\
\hline 8890 & Noctuoidea & Noctuidae & Catocala & fulminea & Braşov & Braşov, Bartolomeu & 1968 & July & 17 & 1 \\
\hline 8890 & Noctuoidea & Noctuidae & Catocala & fulminea & Covasna & Brețcu village & 1982 & July & 23 & 11 \\
\hline 8877 & Noctuoidea & Noctuidae & Catocala & elocata & Hunedoara & Sibişel & 2003 & July & 10 & 1 \\
\hline 8882 & Noctuoidea & Noctuidae & Catocala & promissa & Hunedoara & Sibişel & 2003 & July & 1 & 1 \\
\hline 8969 & Noctuoidea & Noctuidae & Euclidia & glyphica & Braşov & Dealul Lempeş, Sânpetru & 2002 & June & 17 & 1 \\
\hline 8967 & Noctuoidea & Noctuidae & Euclidia & $m i$ & Braşov & Tâmpa, Braşov & 1968 & May & 16 & 1 \\
\hline 8967 & Noctuoidea & Noctuidae & Euclidia & $m i$ & Braşov & Tâmpa, Braşov & 1980 & June & 21 & 1 \\
\hline 8967 & Noctuoidea & Noctuidae & Euclidia & $m i$ & Braşov & Tâmpa, Braşov & 1981 & May & 29 & 1 \\
\hline 10475 & Noctuoidea & Noctuidae & Miltochrista & miniata & Sibiu & Richiria & 1989 & July & 20 & 1 \\
\hline 10475 & Noctuoidea & Noctuidae & Miltochrista & miniata & Hunedoara & Sibişel & 2003 & June & 23 & 2 \\
\hline 10477 & Noctuoidea & Noctuidae & Cybosia & mesomella & Braşov & Pădurea Bogata, Hoghiz & 1998 & July & 11 & 1 \\
\hline 10477 & Noctuoidea & Noctuidae & Cybosia & mesomella & Covasna & Vâlcele & 1980 & July & 25 & 1 \\
\hline 10477 & Noctuoidea & Noctuidae & Cybosia & mesomella & Covasna & Vâlcele & 1981 & June & 19 & 1 \\
\hline 10477 & Noctuoidea & Noctuidae & Cybosia & mesomella & Covasna & Breţcu village & 1982 & July & 23 & 1 \\
\hline 10483 & Noctuoidea & Noctuidae & Atolmis & rubricollis & Braşov & Poiana Braşov, $1000 \mathrm{~m}$. & 1972 & July & 6 & 1 \\
\hline 10483 & Noctuoidea & Noctuidae & Atolmis & rubricollis & Covasna & Breţcu village & 1982 & July & 23 & 1 \\
\hline 10483 & Noctuoidea & Noctuidae & Atolmis & rubricollis & Hunedoara & Sibişel & 2003 & July & 3 & 1 \\
\hline 10485 & Noctuoidea & Noctuidae & Lithosia & quadra & Covasna & Vâlcele & 2003 & August & 5 & 2 \\
\hline 10485 & Noctuoidea & Noctuidae & Lithosia & quadra & Hunedoara & Sibişel & 1999 & June & 19 & 1 \\
\hline 10487 & Noctuoidea & Noctuidae & Eilema & depressa & Braşov & Poiana Braşov, $1000 \mathrm{~m}$. & 1969 & August & 6 & 1 \\
\hline 10487 & Noctuoidea & Noctuidae & Eilema & depressa & Hunedoara & Sibişel & 2000 & July & 10 & 1 \\
\hline 10488 & Noctuoidea & Noctuidae & Eilema & griseola & Hunedoara & Sibişel & 1999 & August & 29 & 1 \\
\hline 10488 & Noctuoidea & Noctuidae & Eilema & griseola & Hunedoara & Sibişel & 2001 & July & 10 & 1 \\
\hline 10489 & Noctuoidea & Noctuidae & Eilema & lurideola & Sibiu & Richiria & 1993 & July & 13 & 1 \\
\hline 10490 & Noctuoidea & Noctuidae & Eilema & complana & Covasna & Vâlcele & 2003 & August & 5 & 2 \\
\hline 10490 & Noctuoidea & Noctuidae & Eilema & complana & Hunedoara & Sibişel & 2001 & July & 10 & 2 \\
\hline 10493 & Noctuoidea & Noctuidae & Eilema & caniola & Braşov & Cheile Râşnoavei, Râşnov & 1981 & September & 2 & 1 \\
\hline 10499 & Noctuoidea & Noctuidae & Eilema & sororcula & Braşov & Dealul Lempeş, Sânpetru & 2007 & May & 29 & 1 \\
\hline
\end{tabular}




\begin{tabular}{|c|c|c|c|c|c|c|c|c|c|c|}
\hline $\begin{array}{c}\text { K \& R } \\
\text { LIST NR. }\end{array}$ & SUPERFAMILY & FAMILY & GENUS & SPECIES & COUNTY & LOCALITY & YEAR & MONTH & \multicolumn{2}{|c|}{ DAY NR. } \\
\hline 10521 & Noctuoidea & Noctuidae & Dysauxes & ancilla & Covasna & Breţcu village & 1982 & July & 23 & 1 \\
\hline 10550 & Noctuoidea & Noctuidae & Phragmatobia & fuliginosa & Hunedoara & Sibişel & 2003 & July & 2 & 1 \\
\hline 10566 & Noctuoidea & Noctuidae & Spilarctia & lutea & Hunedoara & Sibişel & 2003 & June & 27 & 1 \\
\hline 10572 & Noctuoidea & Noctuidae & Diaphora & mendica & Braşov & Pietrele lui Solomon, Braşov & 1981 & June & 1 & 1 \\
\hline 10583 & Noctuoidea & Noctuidae & Diacrisia & sannio & Braşov & Drăuşeni & 1969 & August & 17 & 1 \\
\hline 10557 & Noctuoidea & Noctuidae & Arctia & plantaginis & Braşov & Dealul Bunloc, Săcele & 1969 & July & 16 & 2 \\
\hline 10557 & Noctuoidea & Noctuidae & Arctia & plantaginis & Braşov & Muntele Piatra Mare, 1500-2000 m. & 1982 & July & 20 & 1 \\
\hline 10585 & Noctuoidea & Noctuidae & Arctia & aulica & Braşov & Tâmpa, Braşov & 1981 & May & 29 & 4 \\
\hline 10603 & Noctuoidea & Noctuidae & Callimorpha & dominula & Sibiu & Richiria & 1989 & July & 20 & 1 \\
\hline 10605 & Noctuoidea & Noctuidae & Euplagia & quadripunctaria & Braşov & Dealul Bunloc, Săcele & 1969 & July & 16 & 1 \\
\hline 10605 & Noctuoidea & Noctuidae & Euplagia & quadripunctaria & Covasna & Breţcu village & 1982 & July & 23 & 1 \\
\hline 10605 & Noctuoidea & Noctuidae & Euplagia & quadripunctaria & Hunedoara & Sibişel & 2001 & August & 24 & 1 \\
\hline 10605 & Noctuoidea & Noctuidae & Euplagia & quadripunctaria & Hunedoara & Costeşti & 1991 & July & 8 & 1 \\
\hline 10416 & Noctuoidea & Noctuidae & Arctornis & l-nigrum & Hunedoara & Sibişel & 2003 & June & 27 & 1 \\
\hline 10416 & Noctuoidea & Noctuidae & Arctornis & l-nigrum & Hunedoara & Sibişel & 2003 & July & 5 & 1 \\
\hline 10375 & Noctuoidea & Noctuidae & Lymantria & monacha & Covasna & Vâlcele & 2003 & August & 5 & 4 \\
\hline 10375 & Noctuoidea & Noctuidae & Lymantria & monacha & Hunedoara & Sibişel & 2003 & June & 27 & 1 \\
\hline 10405 & Noctuoidea & Noctuidae & Euproctis & chrysorrhoea & Braşov & Tâmpa, Braşov & 1991 & July & 17 & 1 \\
\hline 10406 & Noctuoidea & Noctuidae & Sphrageidus & similis & Covasna & Vâlcele & 2003 & August & 5 & 2 \\
\hline 10406 & Noctuoidea & Noctuidae & Sphrageidus & similis & Hunedoara & Sibişel & 2001 & July & 12 & 1 \\
\hline 10406 & Noctuoidea & Noctuidae & Sphrageidus & similis & Hunedoara & Sibişel & 2001 & July & 15 & 1 \\
\hline 10406 & Noctuoidea & Noctuidae & Sphrageidus & similis & Hunedoara & Sibişel & 2001 & August & 21 & 1 \\
\hline 10387 & Noctuoidea & Noctuidae & Calliteara & pudibunda & Braşov & Braşov, Bartolomeu & 1967 & May & 27 & 1 \\
\hline 10387 & Noctuoidea & Noctuidae & Calliteara & pudibunda & Hunedoara & Sibişel & 1999 & June & 7 & 1 \\
\hline 10387 & Noctuoidea & Noctuidae & Calliteara & pudibunda & Hunedoara & Sibişel & 1999 & June & 8 & 4 \\
\hline 10392 & Noctuoidea & Noctuidae & Gynaephora & fascelina & Braşov & Homorod & 1969 & August & 16 & 1 \\
\hline 10408 & Noctuoidea & Noctuidae & Penthophera & morio & Braşov & Teliu, 1500 m., munţii Întorsurii & 1978 & July & 2 & 1 \\
\hline 10408 & Noctuoidea & Noctuidae & Penthophera & morio & Braşov & Dealul Lempeş, Sânpetru & 2002 & June & 15 & 1 \\
\hline 10372 & Noctuoidea & Noctuidae & Colocasia & coryli & Braşov & Sâmbăta de Sus & 2010 & August & 1 & 1 \\
\hline 10372 & Noctuoidea & Noctuidae & Colocasia & coryli & Hunedoara & Sibişel & 1999 & August & 1 & 1 \\
\hline 8789 & Noctuoidea & Noctuidae & Craniophora & ligustri & Hunedoara & Sibişel & 2000 & July & 31 & 1 \\
\hline 8789 & Noctuoidea & Noctuidae & Craniophora & ligustri & Hunedoara & Sibişel & 2001 & August & 24 & 1 \\
\hline
\end{tabular}




\begin{tabular}{|c|c|c|c|c|c|c|c|c|c|c|}
\hline $\begin{array}{c}\text { K \& R } \\
\text { LIST NR. }\end{array}$ & SUPERFAMILY & FAMILY & GENUS & SPECIES & COUNTY & LOCALITY & YEAR & MONTH & \multicolumn{2}{|c|}{ DAY NR. } \\
\hline 8772 & Noctuoidea & Noctuidae & Moma & alpium & Hunedoara & Sibişel & 1999 & June & 17 & 2 \\
\hline 8772 & Noctuoidea & Noctuidae & Moma & alpium & Hunedoara & Sibişel & 2003 & June & 28 & 1 \\
\hline 8774 & Noctuoidea & Noctuidae & Acronicta & alni & Covasna & Brețcu village & 1982 & July & 23 & 1 \\
\hline 8776 & Noctuoidea & Noctuidae & Acronicta & tridens & Braşov & Braşov, Bartolomeu & 1969 & July & 31 & 1 \\
\hline 8776 & Noctuoidea & Noctuidae & Acronicta & tridens & Hunedoara & Sibişel & 2001 & August & 21 & 1 \\
\hline 8776 & Noctuoidea & Noctuidae & Acronicta & tridens & Hunedoara & Sibişel & 2001 & August & 24 & 1 \\
\hline 8776 & Noctuoidea & Noctuidae & Acronicta & tridens & Hunedoara & Sibişel & 2002 & July & 13 & 1 \\
\hline 8781 & Noctuoidea & Noctuidae & Acronicta & strigosa & Covasna & Breţcu village & 1982 & July & 23 & 1 \\
\hline 8787 & Noctuoidea & Noctuidae & Acronicta & rumicis & Hunedoara & Sibişel & 1999 & June & 12 & 1 \\
\hline 8787 & Noctuoidea & Noctuidae & Acronicta & rumicis & Hunedoara & Sibişel & 1999 & July & 28 & 1 \\
\hline 8787 & Noctuoidea & Noctuidae & Acronicta & rumicis & Hunedoara & Sibişel & 1999 & August & 1 & 1 \\
\hline 8787 & Noctuoidea & Noctuidae & Acronicta & rumicis & Hunedoara & Sibişel & 2000 & August & 14 & 1 \\
\hline 8779 & Noctuoidea & Noctuidae & Acronicta & leporina & Hunedoara & Sibişel & 2000 & August & 11 & 1 \\
\hline 8780 & Noctuoidea & Noctuidae & Acronicta & megacephala & Braşov & Braşov, Bartolomeu & 1982 & June & 14 & 1 \\
\hline 8780 & Noctuoidea & Noctuidae & Acronicta & megacephala & Hunedoara & Sibişel & 1999 & August & 13 & 1 \\
\hline 8780 & Noctuoidea & Noctuidae & Acronicta & megacephala & Hunedoara & Sibişel & 2001 & August & 21 & 1 \\
\hline 9091 & Noctuoidea & Noctuidae & Abrostola & tripartita & Hunedoara & Sibişel & 1999 & June & 5 & 1 \\
\hline 9091 & Noctuoidea & Noctuidae & Abrostola & tripartita & Hunedoara & Sibişel & 1999 & July & 22 & 2 \\
\hline 9091 & Noctuoidea & Noctuidae & Abrostola & tripartita & Hunedoara & Sibişel & 2003 & July & 1 & 1 \\
\hline 9092 & Noctuoidea & Noctuidae & Abrostola & asclepiadis & Hunedoara & Sibişel & 1999 & June & 10 & 1 \\
\hline 9049 & Noctuoidea & Noctuidae & Diachrysia & chryson & Hunedoara & Sibiş̧el & 2000 & August & 28 & 1 \\
\hline 9046 & Noctuoidea & Noctuidae & Diachrysia & stenochrysis & Braşov & Braşov, Bartolomeu & 1969 & August & 2 & 2 \\
\hline 9027 & Noctuoidea & Noctuidae & Euchalcia & variabilis & Hunedoara & Sibişel & 2000 & June & 4 & 1 \\
\hline 9056 & Noctuoidea & Noctuidae & Autographa & gamma & Braşov & Braşov, Bartolomeu & 1969 & August & 2 & 1 \\
\hline 9059 & Noctuoidea & Noctuidae & Autographa & pulchrina & Braşov & Braşov, Bartolomeu & 1967 & August & 24 & 1 \\
\hline 9059 & Noctuoidea & Noctuidae & Autographa & pulchrina & Hunedoara & Sibişel & 1999 & July & 22 & 1 \\
\hline 9059 & Noctuoidea & Noctuidae & Autographa & pulchrina & Hunedoara & Sibişel & 1999 & August & 2 & 1 \\
\hline 9059 & Noctuoidea & Noctuidae & Autographa & pulchrina & Hunedoara & Sibişel & 2002 & July & 24 & 1 \\
\hline 9061 & Noctuoidea & Noctuidae & Autographa & jota & Covasna & Breţcu village & 1982 & July & 23 & 3 \\
\hline 9062 & Noctuoidea & Noctuidae & Autographa & bractea & Braşov & Săcele & 1982 & July & 25 & 1 \\
\hline 9053 & Noctuoidea & Noctuidae & Plusia & festucae & Braşov & Braşov, Bartolomeu & 1969 & August & 2 & 1 \\
\hline 9114 & Noctuoidea & Noctuidae & Deltote & pygarga & Braşov & Pădurea Bogata, Hoghiz & 1998 & July & 11 & 1 \\
\hline
\end{tabular}




\begin{tabular}{|c|c|c|c|c|c|c|c|c|c|c|}
\hline $\begin{array}{c}\text { K \& R } \\
\text { LIST NR. }\end{array}$ & SUPERFAMILY & FAMILY & GENUS & SPECIES & COUNTY & LOCALITY & YEAR & MONTH & \multicolumn{2}{|c|}{ DAY NR. } \\
\hline 9114 & Noctuoidea & Noctuidae & Deltote & pygarga & Braşov & Pădurea Bogata, Hoghiz & 2002 & June & 10 & 1 \\
\hline 9114 & Noctuoidea & Noctuidae & Deltote & pygarga & Hunedoara & Sibişel & 1999 & June & 9 & 1 \\
\hline 9114 & Noctuoidea & Noctuidae & Deltote & pygarga & Hunedoara & Sibişel & 2003 & June & 23 & 3 \\
\hline 9116 & Noctuoidea & Noctuidae & Deltote & deceptoria & Covasna & Vâlcele & 1981 & June & 19 & 2 \\
\hline 10423 & Noctuoidea & Noctuidae & Meganola & strigula & Hunedoara & Sibișel & 2001 & August & 20 & 1 \\
\hline 10427 & Noctuoidea & Noctuidae & Nola & cucullatella & Hunedoara & Sibişel & 2001 & July & 10 & 1 \\
\hline 10427 & Noctuoidea & Noctuidae & Nola & cucullatella & Hunedoara & Sibişel & 2003 & June & 23 & 2 \\
\hline 10451 & Noctuoidea & Noctuidae & Pseudoips & prasinana & Braşov & Dealul Bunloc, Săcele & 1991 & July & 15 & 1 \\
\hline 10456 & Noctuoidea & Noctuidae & Earias & clorana & Hunedoara & Sibişel & 2003 & June & 28 & 1 \\
\hline 9097 & Noctuoidea & Noctuidae & Acontia & trabealis & Braşov & Dealul Lempeş, Sânpetru & 2002 & June & 15 & 3 \\
\hline 8958 & Noctuoidea & Noctuidae & Aedia & funesta & Hunedoara & Sibişel & 2001 & July & 10 & 1 \\
\hline 9199 & Noctuoidea & Noctuidae & Cucullia & umbratica & Braşov & Braşov, Bartolomeu & 1969 & June & 11 & 1 \\
\hline 9307 & Noctuoidea & Noctuidae & Amphipyra & pyramidea & Braşov & Braşov, Bartolomeu & 1969 & September & 2 & 1 \\
\hline 9307 & Noctuoidea & Noctuidae & Amphipyra & pyramidea & Braşov & Braşov & 1991 & July & 17 & 1 \\
\hline 9307 & Noctuoidea & Noctuidae & Amphipyra & pyramidea & Hunedoara & Sibişel & 2003 & June & 22 & 3 \\
\hline 9372 & Noctuoidea & Noctuidae & Pyrrhia & umbra & Covasna & Breţcu village & 1982 & July & 23 & 1 \\
\hline 9358 & Noctuoidea & Noctuidae & Protoschinia & scutosa & Braşov & Dealul Lempeş, Sânpetru & 1979 & July & 29 & 1 \\
\hline 9358 & Noctuoidea & Noctuidae & Protoschinia & scutosa & Braşov & Braşov & 2010 & August & 6 & 1 \\
\hline 9367 & Noctuoidea & Noctuidae & Heliothis & peltigera & Braşov & Poiana Braşov, $1000 \mathrm{~m}$. & 1969 & July & 31 & 1 \\
\hline 9367 & Noctuoidea & Noctuidae & Heliothis & peltigera & Braşov & Drăuşeni & 1979 & July & 28 & 1 \\
\hline 9364 & Noctuoidea & Noctuidae & Heliothis & viriplaca & Braşov & Drăuşeni & 1969 & August & 1 & 1 \\
\hline 9364 & Noctuoidea & Noctuidae & Heliothis & viriplaca & Hunedoara & Sibişel & 2001 & July & 10 & 1 \\
\hline 9370 & Noctuoidea & Noctuidae & Helicoverpa & armigera & Hunedoara & Sibişel & 2001 & August & 20 & 1 \\
\hline 9520 & Noctuoidea & Noctuidae & Callopistria & juventina & Hunedoara & Sibişel & 1999 & July & 21 & 1 \\
\hline 8801 & Noctuoidea & Noctuidae & Cryphia & algae & Hunedoara & Sibişel & 2001 & August & 18 & 1 \\
\hline 8801 & Noctuoidea & Noctuidae & Cryphia & algae & Hunedoara & Sibişel & 2001 & September & 1 & 3 \\
\hline 8801 & Noctuoidea & Noctuidae & Cryphia & algae & Hunedoara & Sibişel & 2002 & July & 19 & 1 \\
\hline 8806 & Noctuoidea & Noctuidae & Bryophila & ereptricula & Hunedoara & Sibişel & 2001 & August & 21 & 1 \\
\hline 8806 & Noctuoidea & Noctuidae & Bryophila & ereptricula & Hunedoara & Sibişel & 2003 & July & 1 & 1 \\
\hline 8810 & Noctuoidea & Noctuidae & Bryophila & raptricula & Braşov & Braşov, Bartolomeu & 1969 & July & 17 & 1 \\
\hline 9430 & Noctuoidea & Noctuidae & Caradrina & selini & Hunedoara & Sibişel & 2001 & July & 10 & 1 \\
\hline 9433 & Noctuoidea & Noctuidae & Caradrina & clavipalpis & Braşov & Poiana Braşov, $1000 \mathrm{~m}$. & 2003 & September & 14 & 1 \\
\hline
\end{tabular}




\begin{tabular}{|c|c|c|c|c|c|c|c|c|c|c|}
\hline $\begin{array}{c}\text { K \& R } \\
\text { LIST NR. }\end{array}$ & SUPERFAMILY & FAMILY & GENUS & SPECIES & COUNTY & LOCALITY & YEAR & MONTH & \multicolumn{2}{|c|}{ DAY NR. } \\
\hline 9433 & Noctuoidea & Noctuidae & Caradrina & clavipalpis & Hunedoara & Sibişel & 2001 & August & 26 & 1 \\
\hline 9450 & Noctuoidea & Noctuidae & Hoplodrina & blanda & Hunedoara & Sibişel & 2003 & June & 28 & 1 \\
\hline 9450 & Noctuoidea & Noctuidae & Hoplodrina & blanda & Hunedoara & Sibişel & 2003 & June & 29 & 1 \\
\hline 9450 & Noctuoidea & Noctuidae & Hoplodrina & blanda & Hunedoara & Sibişel & 2003 & July & 1 & 1 \\
\hline 9456 & Noctuoidea & Noctuidae & Charanyca & trigrammica & Braşov & Braşov, Bartolomeu & 1969 & June & 7 & 1 \\
\hline 9455 & Noctuoidea & Noctuidae & Charanyca & trigrammica & Hunedoara & Sibişel & 1999 & June & 5 & 1 \\
\hline 9483 & Noctuoidea & Noctuidae & Rusina & ferruginea & Hunedoara & Sibişel & 1999 & June & 18 & 1 \\
\hline 9483 & Noctuoidea & Noctuidae & Rusina & ferruginea & Hunedoara & Sibişel & 2000 & August & 12 & 1 \\
\hline 9501 & Noctuoidea & Noctuidae & Trachea & atriplicis & Hunedoara & Sibişel & 1999 & July & 29 & 1 \\
\hline 9496 & Noctuoidea & Noctuidae & Thalpophila & matura & Hunedoara & Sibişel & 2000 & August & 9 & 1 \\
\hline 9508 & Noctuoidea & Noctuidae & Hурра & rectilinea & Hunedoara & Sibişel & 1999 & June & 20 & 1 \\
\hline 9515 & Noctuoidea & Noctuidae & Actinotia & polyodon & Covasna & Vâlcele & 2003 & August & 5 & 2 \\
\hline 9503 & Noctuoidea & Noctuidae & Euplexia & lucipara & Hunedoara & Sibişel & 2000 & August & 9 & 1 \\
\hline 9531 & Noctuoidea & Noctuidae & Enargia & paleacea & Hunedoara & Sibişel & 2002 & July & 25 & 1 \\
\hline 9531 & Noctuoidea & Noctuidae & Enargia & paleacea & Hunedoara & Costeşti & 2013 & September & 28 & 1 \\
\hline 9527 & Noctuoidea & Noctuidae & Ipimorpha & retusa & Hunedoara & Sibişel & 2000 & August & 12 & 1 \\
\hline 9527 & Noctuoidea & Noctuidae & Ipimorpha & retusa & Hunedoara & Sibişel & 2003 & August & 7 & 1 \\
\hline 9528 & Noctuoidea & Noctuidae & Ipimorpha & subtusa & Covasna & Breţcu village & 1982 & July & 23 & 1 \\
\hline 9528 & Noctuoidea & Noctuidae & Ipimorpha & subtusa & Hunedoara & Sibişel & 2000 & August & 8 & 1 \\
\hline 9550 & Noctuoidea & Noctuidae & Cosmia & trapezina & Hunedoara & Sibişel & 1999 & June & 15 & 1 \\
\hline 9549 & Noctuoidea & Noctuidae & Cosmia & pyralina & Covasna & Breţcu village & 1982 & July & 23 & 1 \\
\hline 9562 & Noctuoidea & Noctuidae & Tiliacea & citrago & Braşov & Braşov & 2003 & September & 14 & 1 \\
\hline 9559 & Noctuoidea & Noctuidae & Xanthia & icteritia & Covasna & Breţcu village & 1978 & October & 4 & 1 \\
\hline 9566 & Noctuoidea & Noctuidae & Sunira & circellaris & Braşov & Dealul Bunloc, Săcele & 1982 & September & 29 & 1 \\
\hline 9566 & Noctuoidea & Noctuidae & Sunira & circellaris & Braşov & Pietrele lui Solomon, Braşov & 1982 & September & 24 & 1 \\
\hline 9642 & Noctuoidea & Noctuidae & Brachylomia & viminalis & Covasna & Breţcu village & 1982 & July & 23 & 2 \\
\hline 9642 & Noctuoidea & Noctuidae & Brachylomia & viminalis & Hunedoara & Sibişel & 2003 & June & 28 & 1 \\
\hline 9642 & Noctuoidea & Noctuidae & Brachylomia & viminalis & Hunedoara & Sibişel & 2003 & June & 30 & 1 \\
\hline 9642 & Noctuoidea & Noctuidae & Brachylomia & viminalis & Hunedoara & Sibişel & 2003 & July & 1 & 1 \\
\hline 9658 & Noctuoidea & Noctuidae & Lithophane & socia & Braşov & Braşov, Bartolomeu & 1967 & September & 2 & 1 \\
\hline 9658 & Noctuoidea & Noctuidae & Lithophane & socia & Braşov & Braşov, Bartolomeu & 1969 & May & 19 & 1 \\
\hline 9670 & Noctuoidea & Noctuidae & Xylena & vetusta & Braşov & Braşov, Bartolomeu & 1969 & April & 4 & 1 \\
\hline
\end{tabular}




\begin{tabular}{|c|c|c|c|c|c|c|c|c|c|c|}
\hline $\begin{array}{c}\text { K \& R } \\
\text { LIST NR. }\end{array}$ & SUPERFAMILY & FAMILY & GENUS & SPECIES & COUNTY & LOCALITY & YEAR & MONTH & \multicolumn{2}{|c|}{ DAY NR. } \\
\hline 9596 & Noctuoidea & Noctuidae & Eupsilia & transversa & Hunedoara & Costeşti & 2013 & September & 28 & 1 \\
\hline 9536 & Noctuoidea & Noctuidae & Parastichtis & suspecta & Braşov & Dealul Bunloc, Săcele & 1981 & July & 29 & 1 \\
\hline 9537 & Noctuoidea & Noctuidae & Apterogenum & ypsillon & Hunedoara & Sibişel & 2003 & June & 25 & 1 \\
\hline 9537 & Noctuoidea & Noctuidae & Apterogenum & ypsillon & Hunedoara & Sibişel & 2003 & June & 30 & 1 \\
\hline 9458 & Noctuoidea & Noctuidae & Atypha & pulmonaris & Hunedoara & Sibişel & 2003 & June & 22 & 1 \\
\hline 9741 & Noctuoidea & Noctuidae & Mniotype & adusta & Hunedoara & Sibişel & 1999 & July & 30 & 1 \\
\hline 9741 & Noctuoidea & Noctuidae & Mniotype & adusta & Hunedoara & Sibişel & 2000 & August & 27 & 1 \\
\hline 9738 & Noctuoidea & Noctuidae & Mniotype & satura & Braşov & Homorod & 1969 & August & 16 & 1 \\
\hline 9738 & Noctuoidea & Noctuidae & Mniotype & satura & Hunedoara & Sibişel & 2000 & August & 19 & 1 \\
\hline 9738 & Noctuoidea & Noctuidae & Mniotype & satura & Hunedoara & Sibişel & 2000 & August & 28 & 1 \\
\hline 9738 & Noctuoidea & Noctuidae & Mniotype & satura & Hunedoara & Sibişel & 2001 & August & 23 & 2 \\
\hline 9738 & Noctuoidea & Noctuidae & Mniotype & satura & Hunedoara & Sibişel & 2002 & July & 18 & 1 \\
\hline 9834 & Noctuoidea & Noctuidae & Hydraecia & micacea & Covasna & Breţcu village & 1982 & July & 23 & 1 \\
\hline 9829 & Noctuoidea & Noctuidae & Amphipoea & fucosa & Covasna & Brețcu village & 1982 & July & 23 & 1 \\
\hline 9828 & Noctuoidea & Noctuidae & Amphipoea & oculea & Covasna & Breţcu village & 1982 & July & 23 & 1 \\
\hline 9828 & Noctuoidea & Noctuidae & Amphipoea & oculea & Hunedoara & Sibişel & 1999 & August & 13 & 1 \\
\hline 9828 & Noctuoidea & Noctuidae & Amphipoea & oculea & Hunedoara & Sibişel & 2000 & August & 13 & 1 \\
\hline 9801 & Noctuoidea & Noctuidae & Luperina & testacea & Braşov & Braşov, Bartolomeu & 1969 & August & 24 & 1 \\
\hline 9859 & Noctuoidea & Noctuidae & Nonagria & typhae & Braşov & Braşov, Bartolomeu & 1969 & September & 23 & 1 \\
\hline 9756 & Noctuoidea & Noctuidae & Apamea & epomidion & Braşov & Braşov & 1991 & July & 17 & 1 \\
\hline 9770 & Noctuoidea & Noctuidae & Apamea & anceps & Braşov & Drăuşeni & 1981 & June & 4 & 1 \\
\hline 9774 & Noctuoidea & Noctuidae & Apamea & scolopacina & Hunedoara & Sibişel & 2003 & July & 2 & 1 \\
\hline 9748 & Noctuoidea & Noctuidae & Apamea & monoglypha & Hunedoara & Sibişel & 2000 & August & 12 & 1 \\
\hline 9748 & Noctuoidea & Noctuidae & Apamea & monoglypha & Hunedoara & Sibişel & 2001 & August & 18 & 2 \\
\hline $9776 a$ & Noctuoidea & Noctuidae & Apamea & syriaca & Braşov & Braşov & 1982 & June & 11 & 1 \\
\hline 9752 & Noctuoidea & Noctuidae & Apamea & lithoxylaea & Braşov & Braşov, Bartolomeu & 1967 & July & 16 & 1 \\
\hline 9786 & Noctuoidea & Noctuidae & Mesoligia & furuncula & Braşov & Braşov, Bartolomeu & 1968 & July & 20 & 1 \\
\hline 9780 & Noctuoidea & Noctuidae & Oligia & strigilis & Braşov & Homorod & 1980 & June & 22 & 1 \\
\hline 9780 & Noctuoidea & Noctuidae & Oligia & strigilis & Braşov & Dealul Lempeş, Sânpetru & 2002 & June & 15 & 1 \\
\hline 9780 & Noctuoidea & Noctuidae & Oligia & strigilis & Braşov & Braşov, Bartolomeu & 2007 & May & 27 & 1 \\
\hline 9780 & Noctuoidea & Noctuidae & Oligia & strigilis & Hunedoara & Sibişel & 2003 & June & 23 & 2 \\
\hline 9782 & Noctuoidea & Noctuidae & Oligia & latruncula & Hunedoara & Sibişel & 1999 & June & 3 & 1 \\
\hline
\end{tabular}




\begin{tabular}{|c|c|c|c|c|c|c|c|c|c|c|}
\hline $\begin{array}{c}\text { K \& R } \\
\text { LIST NR. }\end{array}$ & SUPERFAMILY & FAMILY & GENUS & SPECIES & COUNTY & LOCALITY & YEAR & MONTH & \multicolumn{2}{|c|}{ DAY NR. } \\
\hline 9782 & Noctuoidea & Noctuidae & Oligia & latruncula & Hunedoara & Sibişel & 2003 & June & 25 & 1 \\
\hline 9782 & Noctuoidea & Noctuidae & Oligia & latruncula & Hunedoara & Sibişel & 2003 & July & 9 & 1 \\
\hline 9781 & Noctuoidea & Noctuidae & Oligia & versicolor & Braşov & Braşov, Bartolomeu & 1969 & June & 5 & 1 \\
\hline 9781 & Noctuoidea & Noctuidae & Oligia & versicolor & Braşov & Braşov, Bartolomeu & 2007 & May & 27 & 1 \\
\hline 9781 & Noctuoidea & Noctuidae & Oligia & versicolor & Hunedoara & Sibişel & 1999 & June & 3 & 1 \\
\hline 9781 & Noctuoidea & Noctuidae & Oligia & versicolor & Hunedoara & Sibişel & 2003 & June & 28 & 1 \\
\hline 9781 & Noctuoidea & Noctuidae & Oligia & versicolor & Hunedoara & Sibişel & 2003 & July & 2 & 2 \\
\hline 9895 & Noctuoidea & Noctuidae & Anarta & trifolii & Hunedoara & Sibişel & 2003 & July & 1 & 1 \\
\hline 9993 & Noctuoidea & Noctuidae & Polia & nebulosa & Covasna & Breţcu village & 1982 & July & 23 & 1 \\
\hline 9993 & Noctuoidea & Noctuidae & Polia & nebulosa & Hunedoara & Sibişel & 1999 & June & 8 & 1 \\
\hline 9993 & Noctuoidea & Noctuidae & Polia & nebulosa & Hunedoara & Sibişel & 1999 & June & 20 & 1 \\
\hline 10068 & Noctuoidea & Noctuidae & Pachetra & sagittigera & Hunedoara & Sibişel & 1999 & July & 28 & 1 \\
\hline 9912 & Noctuoidea & Noctuidae & Lacanobia & w-latinum & Braşov & Braşov & 1982 & May & 29 & 1 \\
\hline 9912 & Noctuoidea & Noctuidae & Lacanobia & w-latinum & Hunedoara & Sibişel & 1999 & June & 14 & 1 \\
\hline 9912 & Noctuoidea & Noctuidae & Lacanobia & w-latinum & Hunedoara & Sibişel & 1999 & August & 2 & 1 \\
\hline 9919 & Noctuoidea & Noctuidae & Lacanobia & contigua & Hunedoara & Sibişel & 1999 & June & 6 & 1 \\
\hline 9919 & Noctuoidea & Noctuidae & Lacanobia & contigua & Hunedoara & Sibişel & 1999 & June & 9 & 1 \\
\hline 9919 & Noctuoidea & Noctuidae & Lacanobia & contigua & Hunedoara & Sibişel & 1999 & June & 18 & 1 \\
\hline 9919 & Noctuoidea & Noctuidae & Lacanobia & contigua & Hunedoara & Sibişel & 1999 & August & 15 & 1 \\
\hline 9919 & Noctuoidea & Noctuidae & Lacanobia & contigua & Hunedoara & Sibişel & 2000 & August & 20 & 1 \\
\hline 9917 & Noctuoidea & Noctuidae & Lacanobia & oleracea & Hunedoara & Sibişel & 2002 & July & 22 & 1 \\
\hline 9984 & Noctuoidea & Noctuidae & Melanchra & persicariae & Hunedoara & Sibişel & 1999 & June & 9 & 1 \\
\hline 9984 & Noctuoidea & Noctuidae & Melanchra & persicariae & Hunedoara & Sibişel & 2003 & June & 28 & 1 \\
\hline 9984 & Noctuoidea & Noctuidae & Melanchra & persicariae & Hunedoara & Sibişel & 2003 & July & 10 & 1 \\
\hline 9987 & Noctuoidea & Noctuidae & Mamestra & brassicae & Braşov & Braşov, Bartolomeu & 1969 & August & 3 & 1 \\
\hline 9987 & Noctuoidea & Noctuidae & Mamestra & brassicae & Braşov & Braşov, Bartolomeu & 1982 & September & 5 & 1 \\
\hline 9987 & Noctuoidea & Noctuidae & Mamestra & brassicae & Braşov & Braşov & 2010 & August & 6 & 1 \\
\hline 9955 & Noctuoidea & Noctuidae & Sideridis & rivularis & Hunedoara & Sibişel & 1999 & July & 12 & 1 \\
\hline 9955 & Noctuoidea & Noctuidae & Sideridis & rivularis & Hunedoara & Sibişel & 1999 & July & 21 & 1 \\
\hline 9972 & Noctuoidea & Noctuidae & Sideridis & reticulata & Braşov & Braşov, Bartolomeu & 1969 & June & 1 & 1 \\
\hline 9935 & Noctuoidea & Noctuidae & Luteohadena & luteago & Braşov & Braşov, Bartolomeu & 1967 & September & 13 & 1 \\
\hline 9935 & Noctuoidea & Noctuidae & Luteohadena & luteago & Braşov & Braşov, Bartolomeu & 1968 & October & 20 & 1 \\
\hline
\end{tabular}




\begin{tabular}{|c|c|c|c|c|c|c|c|c|c|c|}
\hline $\begin{array}{c}\text { K \& R } \\
\text { LIST NR. }\end{array}$ & SUPERFAMILY & FAMILY & GENUS & SPECIES & COUNTY & LOCALITY & YEAR & MONTH & \multicolumn{2}{|c|}{ DAY NR. } \\
\hline 9927 & Noctuoidea & Noctuidae & Hecatera & dysodea & Braşov & Braşov, Bartolomeu & 1967 & August & 4 & 1 \\
\hline 9927 & Noctuoidea & Noctuidae & Hecatera & dysodea & Hunedoara & Sibişel & 2003 & June & 23 & 1 \\
\hline 9944 & Noctuoidea & Noctuidae & Hadena & albimacula & Hunedoara & Sibişel & 1999 & June & 4 & 1 \\
\hline 10070 & Noctuoidea & Noctuidae & Eriopygides & imbecilla & Braşov & Braşov, Bartolomeu & 1969 & July & 18 & 1 \\
\hline 10062 & Noctuoidea & Noctuidae & Cerapteryx & graminis & Braşov & Dealul Bunloc, Săcele & 1981 & July & 29 & 4 \\
\hline 10062 & Noctuoidea & Noctuidae & Cerapteryx & graminis & Braşov & Valea Gârcinului, Săcele & 1981 & July & 24 & 1 \\
\hline 10062 & Noctuoidea & Noctuidae & Cerapteryx & graminis & Covasna & Breţcu village & 1982 & July & 23 & 5 \\
\hline 10062 & Noctuoidea & Noctuidae & Cerapteryx & graminis & Hunedoara & Sibişel & 2001 & August & 20 & 1 \\
\hline 10007 & Noctuoidea & Noctuidae & Mythimna & pallens & Braşov & Braşov, Bartolomeu & 1969 & June & 3 & 1 \\
\hline 10007 & Noctuoidea & Noctuidae & Mythimna & pallens & Braşov & Braşov, Bartolomeu & 1982 & May & 3 & 1 \\
\hline 10001 & Noctuoidea & Noctuidae & Mythimna & ferrago & Hunedoara & Sibişel & 2000 & August & 12 & 1 \\
\hline 10001 & Noctuoidea & Noctuidae & Mythimna & ferrago & Hunedoara & Sibişel & 2001 & July & 10 & 2 \\
\hline 10019 & Noctuoidea & Noctuidae & Mythimna & congrua & Covasna & Breţcu village & 1982 & July & 23 & 1 \\
\hline 10011 & Noctuoidea & Noctuidae & Leucania & comma & Hunedoara & Sibişel & 2003 & June & 20 & 1 \\
\hline 10044 & Noctuoidea & Noctuidae & Orthosia & cerasi & Braşov & Braşov, Bartolomeu & 1969 & April & 15 & 1 \\
\hline 10048 & Noctuoidea & Noctuidae & Orthosia & gracilis & Braşov & Braşov, Bartolomeu & 1968 & April & 28 & 1 \\
\hline 10351 & Noctuoidea & Noctuidae & Agrotis & segetum & Hunedoara & Sibişel & 1999 & July & 30 & 1 \\
\hline 10350 & Noctuoidea & Noctuidae & Agrotis & clavis & Hunedoara & Sibişel & 2001 & July & 10 & 1 \\
\hline 10348 & Noctuoidea & Noctuidae & Agrotis & exclamationis & Hunedoara & Sibişel & 1999 & June & 13 & 1 \\
\hline 10348 & Noctuoidea & Noctuidae & Agrotis & exclamationis & Hunedoara & Sibișel & 2000 & August & 4 & 1 \\
\hline 10346 & Noctuoidea & Noctuidae & Agrotis & ipsilon & Hunedoara & Sibişel & 2000 & August & 28 & 1 \\
\hline 10084 & Noctuoidea & Noctuidae & Dichagyris & flammatra & Braşov & Braşov, Bartolomeu & 1967 & May & 14 & 1 \\
\hline 10084 & Noctuoidea & Noctuidae & Dichagyris & flammatra & Braşov & Braşov & 2003 & September & 14 & 1 \\
\hline 10086 & Noctuoidea & Noctuidae & Ochropleura & plecta & Hunedoara & Sibişel & 2000 & August & 14 & 1 \\
\hline 10086 & Noctuoidea & Noctuidae & Ochropleura & plecta & Hunedoara & Sibişel & 2002 & July & 11 & 1 \\
\hline 10096 & Noctuoidea & Noctuidae & Noctua & pronuba & Braşov & Homorod & 1980 & June & 23 & 1 \\
\hline 10100 & Noctuoidea & Noctuidae & Noctua & fimbriata & Hunedoara & Sibişel & 2000 & August & 27 & 1 \\
\hline 10103 & Noctuoidea & Noctuidae & Noctua & janthe & Hunedoara & Sibişel & 2001 & August & 20 & 1 \\
\hline 10171 & Noctuoidea & Noctuidae & Graphiphora & augur & Covasna & Breţcu village & 1982 & July & 23 & 2 \\
\hline 10218 & Noctuoidea & Noctuidae & Eugraphe & sigma & Hunedoara & Sibişel & 2003 & June & 22 & 3 \\
\hline 10092 & Noctuoidea & Noctuidae & Diarsia & brunnea & Covasna & Breţcu village & 1982 & July & 23 & 1 \\
\hline 10092 & Noctuoidea & Noctuidae & Diarsia & brunnea & Hunedoara & Sibişel & 1999 & June & 17 & 1 \\
\hline
\end{tabular}




\begin{tabular}{|c|c|c|c|c|c|c|c|c|c|c|}
\hline $\begin{array}{c}\text { K \& R } \\
\text { LIST NR. }\end{array}$ & SUPERFAMILY & FAMILY & GENUS & SPECIES & COUNTY & LOCALITY & YEAR & MONTH & $\overline{D A Y}$ & NR. \\
\hline 10092 & Noctuoidea & Noctuidae & Diarsia & brunnea & Hunedoara & Sibişel & 2000 & July & 10 & 1 \\
\hline 10092 & Noctuoidea & Noctuidae & Diarsia & brunnea & Hunedoara & Sibişel & 2000 & August & 7 & 1 \\
\hline 10089 & Noctuoidea & Noctuidae & Diarsia & mendica & Hunedoara & Sibişel & 2001 & September & 2 & 1 \\
\hline 10178 & Noctuoidea & Noctuidae & Eugnorisma & depuncta & Hunedoara & Sibişel & 2001 & August & 24 & 1 \\
\hline 10199 & Noctuoidea & Noctuidae & Xestia & c-nigrum & Braşov & Braşov, Bartolomeu & 1969 & June & 26 & 1 \\
\hline 10201 & Noctuoidea & Noctuidae & Xestia & triangulum & Hunedoara & Sibişel & 2003 & June & 23 & 1 \\
\hline 10201 & Noctuoidea & Noctuidae & Xestia & triangulum & Hunedoara & Sibişel & 2003 & June & 29 & 3 \\
\hline 10201 & Noctuoidea & Noctuidae & Xestia & triangulum & Hunedoara & Sibişel & 2003 & July & 1 & 2 \\
\hline 10204 & Noctuoidea & Noctuidae & Xestia & baja & Hunedoara & Sibişel & 1999 & August & 17 & 1 \\
\hline 10204 & Noctuoidea & Noctuidae & Xestia & baja & Hunedoara & Sibişel & 2001 & August & 27 & 2 \\
\hline 10204 & Noctuoidea & Noctuidae & Xestia & baja & Hunedoara & Sibişel & 2002 & July & 27 & 1 \\
\hline 10204 & Noctuoidea & Noctuidae & Xestia & baja & Hunedoara & Sibişel & 2002 & July & 30 & 1 \\
\hline 10204 & Noctuoidea & Noctuidae & Xestia & baja & Hunedoara & Sibişel & 2003 & June & 30 & 1 \\
\hline 10206 & Noctuoidea & Noctuidae & Xestia & stigmatica & Hunedoara & Sibişel & 2001 & August & 29 & 3 \\
\hline 10206 & Noctuoidea & Noctuidae & Xestia & stigmatica & Hunedoara & Sibişel & 2003 & July & 1 & 1 \\
\hline 10232 & Noctuoidea & Noctuidae & Anaplectoides & prasina & Braşov & Braşov, Bartolomeu & 1978 & August & 5 & 1 \\
\hline 10232 & Noctuoidea & Noctuidae & Anaplectoides & prasina & Covasna & Breţcu village & 1982 & July & 23 & 7 \\
\hline 10232 & Noctuoidea & Noctuidae & Anaplectoides & prasina & Hunedoara & Sibişel & 1999 & July & 17 & 1 \\
\hline 10232 & Noctuoidea & Noctuidae & Anaplectoides & prasina & Hunedoara & Sibişel & 2002 & July & 27 & 1 \\
\hline
\end{tabular}

